\title{
IDEALS ASSOCIATED TO TWO SEQUENCES AND A MATRIX
}

\author{
ANDREW R. Kustin
}

\begin{abstract}
Let $\mathbf{u}_{1 \times n}, \mathbf{X}_{n \times n}$, and $\mathbf{v}_{n \times 1}$ be matrices of indeterminates, Adj $\mathbf{X}$ be the classical adjoint of $\mathbf{X}$, and $H(n)$ be the ideal $I_{1}(\mathbf{u X})+I_{1}(\mathbf{X} \mathbf{v})+I_{1}(\mathbf{v u}-\operatorname{Adj} \mathbf{X})$. Vasconcelos has conjectured that $H(n)$ is a perfect Gorenstein ideal of grade $2 n$. In this paper, we obtain the minimal free resolution of $H(n)$; and thereby establish Vasconcelos' conjecture.
\end{abstract}

Let $\mathbf{u}_{1 \times n}, \mathbf{X}_{n \times n}$, and $\mathbf{v}_{n \times 1}$ be matrices of indeterminates over a commutative noetherian ring $R_{0}$, and let $H(n)$ be the ideal $I_{1}(\mathbf{u X})+I_{1}(\mathbf{X} \mathbf{v})+I_{1}(\mathbf{v u}-\operatorname{Adj} \mathbf{X})$ of the polynomial ring $R=R_{0}\left[\left\{u_{i}, v_{i}, x_{i j} \mid 1 \leq i, j \leq n\right\}\right]$. Vasconcelos has conjectured, in [10, Conjecture 3.3.1], that the ideal $H(n)$ is a perfect Gorenstein ideal of grade $2 n$. In this paper, we obtain the minimal homogeneous resolution of $R / H(n)$ by free $R$-modules; and thereby establish Vasconcelos' conjecture.

In fact, we produce two resolutions of $R / H(n)$. The complex $\mathbb{F}$ of section 2 is never minimal, but it is relatively straightforward. The complex $\mathbb{M}$ of section 3 is a quotient of $\mathbb{F}$. It is more complicated than $\mathbb{F}$, but it is minimal. The exactness of $\mathbb{F}$ and $\mathbb{M}$ is established in section 4 . In section 5 we consider the singular locus and linkage history of $R / H(n)$; we also consider the algebra structure of $\operatorname{Tor}_{\bullet}^{R}\left(R / H(n), R_{0}\right)$. At the end of the paper we record some open questions about the ring $R / H(n)$.

The arguments of sections 2,3 , and 4 are long, but routine. In section 2 we prove that $\mathbb{F}$ is a complex. In section 3 we split off a split exact subcomplex $\mathbb{N}$ of $\mathbb{F}$. In section 4 we apply the acyclicity lemma and reduce the problem to one involving generic data with the parameter $n$ replaced by $n-1$. The most interesting part of the argument is the discovery of the complex $\mathbb{F}$. This complex is obtained by merging four Koszul complexes:

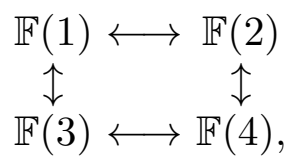

where $\mathbb{F}(1)$ and $\mathbb{F}(4)$ are both Koszul complexes on the entries of $\left[\begin{array}{ll}\mathbf{u} & \mathbf{v}\end{array}, \mathbb{F}(2)\right.$ is the Koszul complex on the entries of $\left[\begin{array}{ll}\mathbf{u} & \mathbf{v}\end{array}\right]$, and $\mathbb{F}(3)$ is the Koszul complex on the entries of $\left[\begin{array}{ll}\mathbf{u} & \mathbf{X} \mathbf{v}\end{array}\right]$. The arrows in $(*)$ represent maps given by the various minors of $\mathbf{X}$.

1991 Mathematics Subject Classification. 13H10, 13D25.

Key words and phrases. Acyclicity lemma, Exterior algebra, Finite free resolution, Gorenstein ideal, Koszul complex, Multilinear algebra, Perfect ideal, Tor-algebra. 


\section{Preliminary results.}

In this paper "ring" means commutative noetherian ring with one. The grade of a proper ideal $I$ in a ring $R$ is the length of the longest regular sequence on $R$ in $I$. The ideal $I$ of $R$ is called perfect if the grade of $I$ is equal to the projective dimension of the $R$-module $R / I$. The grade $g$ ideal $I$ is called Gorenstein if it is perfect and $\operatorname{Ext}_{R}^{g}(R / I, R) \cong R / I$. It follows from Bass [2, Proposition 5.1] that if $I$ is a Gorenstein ideal in a Gorenstein ring $R$, then $R / I$ is also a Gorenstein ring.

Let $R$ be a ring. For any $R$-module $F$, we write $F^{*}=\operatorname{Hom}_{R}(F, R)$. If $f: F \rightarrow G$ is a map of $R$-modules, we define $I_{r}(f)$ to be the image of the map $\bigwedge^{r} F \otimes$ $\left(\bigwedge^{r} G\right)^{*} \rightarrow R$, which is induced by the map $\bigwedge^{r} f: \bigwedge^{r} F \rightarrow \bigwedge^{r} G$.

Definition 1.1. Let $R$ be a commutative ring. If $\mathbf{u}_{1 \times n}, \mathbf{X}_{n \times n}$, and $\mathbf{v}_{n \times 1}$ are matrices with entries from $R$, then $H(\mathbf{u}, \mathbf{X}, \mathbf{v})$ is defined to be the ideal

$$
I_{1}(\mathbf{u X})+I_{1}(\mathbf{X} \mathbf{v})+I_{1}(\mathbf{v u}-\operatorname{Adj} \mathbf{X})
$$

of $R$, where Adj $\mathbf{X}$ is the classical adjoint of $\mathbf{X}$. (In other words, $\mathbf{X} \cdot \operatorname{Adj} \mathbf{X}$ and $\operatorname{Adj} \mathbf{X} \cdot \mathbf{X}$ are both equal to $\operatorname{det} \mathbf{X} \cdot I)$.

Let $R$ be a commutative noetherian ring, and $F$ be a free $R$-module of finite rank. We make much use of the exterior algebras $\Lambda^{\bullet} F$ and $\Lambda^{\bullet} F^{*}$. Each element of $F^{*}$ is a graded derivation on $\bigwedge^{\bullet} F$. In other words,

$$
\alpha_{1}\left(a_{1}^{[1]} \wedge \ldots \wedge a_{1}^{[s]}\right)=\sum_{j}(-1)^{j+1} \alpha_{1}\left(a_{1}^{[j]}\right) \cdot a_{1}^{[1]} \wedge \ldots \wedge \widehat{a_{1}^{[j]}} \wedge \ldots \wedge a_{1}^{[s]} \in \wedge^{j-1} F
$$

for all $\alpha_{1} \in F^{*}$ and $a_{1}^{[j]} \in F$. This action gives rise to the $\bigwedge^{\bullet} F^{*}$-module structure on $\bigwedge^{\bullet} F$. In particular,

$$
\left(\alpha_{1} \wedge \beta_{1}\right)\left(a_{s}\right)=\alpha_{1}\left(\beta_{1}\left(a_{s}\right)\right)
$$

for $\alpha_{1}, \beta_{1} \in F^{*}$ and $a_{s} \in \bigwedge^{s} F$. The $\bigwedge^{\bullet} F$-module structure on $\bigwedge^{\bullet} F^{*}$ is obtained in an analogous manner. In particular, if $a_{i} \in \bigwedge^{i} F$ and $\beta_{j} \in \Lambda^{j} F^{*}$, then

$$
a_{i}\left(\beta_{j}\right) \in \bigwedge^{j-i} F^{*} \quad \text { and } \quad \beta_{j}\left(a_{i}\right) \in \bigwedge^{i-j} F \text {. }
$$

One consequence of these two module structures is that $a_{s}\left(\alpha_{s}\right)=\alpha_{s}\left(a_{s}\right) \in R$ for all $a_{s}$ in $\bigwedge^{s} F$ and $\alpha_{s} \in \bigwedge^{s} F^{*}$. The following well known formulas show more of the interaction between the two module structures.

Proposition 1.2. Let $F$ be a free module over a commutative noetherian ring $R$ and let $a, b \in \bigwedge^{\bullet} F$ and $\gamma \in \bigwedge^{\bullet} F^{*}$ be homogeneous elements.

(a) If $\operatorname{deg} a=1$, then

$$
(a(\gamma))(b)=a \wedge(\gamma(b))+(-1)^{1+\operatorname{deg} \gamma} \gamma(a \wedge b)
$$

(b) If $\gamma \in \bigwedge^{\operatorname{rank} F} F^{*}$, then

$$
(a(\gamma))(b)=(-1)^{\nu}(b(\gamma))(a),
$$

where $\nu=(\operatorname{rank} F-\operatorname{deg} a)(\operatorname{rank} F-\operatorname{deg} b)$.

Note. The value for $\nu$ which is given above is correct. An incorrect value has 
Corollary 1.3. Retain the hypotheses of Proposition 1.2. If $b \in \bigwedge^{\text {rank } F} F$, then $[a(\gamma)](b)$ is equal to $a \wedge \gamma(b)$.

Proof. The proof is by induction on $\operatorname{deg} a$. The case $\operatorname{deg} a=1$ is established in Proposition 1.2 (a). If $a=a_{1} \wedge a^{\prime}$, with $\operatorname{deg} a_{1}=1$, then use the case $\operatorname{deg} a_{1}=1$ and the induction hypothesis to see that

$$
\left[\left(a_{1} \wedge a^{\prime}\right)(\gamma)\right](b)=\left[a_{1}\left(a^{\prime}(\gamma)\right)\right](b)=a_{1} \wedge\left(a^{\prime}(\gamma)\right)(b)=\left(a_{1} \wedge a^{\prime}\right) \wedge \gamma(b)
$$

Remark 1.4. Let $F$ be a free module over a commutative ring $R$. The exterior algebra $\bigwedge^{\bullet} F$ comes equipped with co-multiplication

$$
\Delta: \bigwedge^{\bullet} F \rightarrow \bigwedge^{\bullet} F \otimes \bigwedge^{\bullet} F
$$

Co-multiplication is the algebra map which is induced by the diagonal map $F \rightarrow$ $F \oplus F$. For example, if $a_{1}, a_{1}^{\prime}$, and $a_{1}^{\prime \prime}$ are elements of $\bigwedge^{1} F$, then

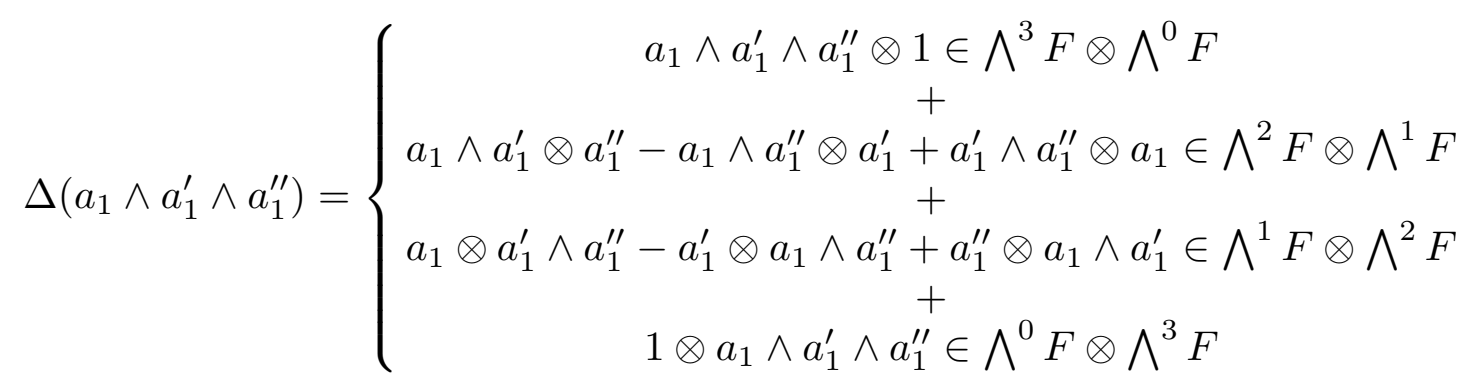

Often, we will use only one graded piece of the co-multiplication map. If $p+q=t$, then we write

$$
\Delta\left(a_{t}\right)=\sum_{j} a_{p}^{[j]} \otimes a_{q}^{[j]}
$$

to mean that the image of $a_{t}$ under the composition

$$
\bigwedge^{t} F \stackrel{\text { inclusion }}{\longrightarrow} \bigwedge^{\bullet} F \stackrel{\Delta}{\longrightarrow} \bigwedge^{\bullet} F \otimes \bigwedge^{\bullet} F \stackrel{\text { projection }}{\longrightarrow} \bigwedge^{p} F \otimes \bigwedge^{q} F
$$

is $\sum_{j} a_{p}^{[j]} \wedge a_{q}^{[j]}$. In particular, if $p=1, q=2$, and $a_{3}=a_{1} \wedge a_{1}^{\prime} \wedge a_{1}^{\prime \prime}$, then

$$
\Delta\left(a_{3}\right)=\sum_{j=1}^{3} a_{1}^{[j]} \otimes a_{2}^{[j]}, \quad \text { where }
$$

$a_{1}^{[1]}=a_{1}, a_{2}^{[1]}=a_{1}^{\prime} \wedge a_{1}^{\prime \prime}, \quad a_{1}^{[2]}=-a_{1}^{\prime}, a_{2}^{[2]}=a_{1} \wedge a_{1}^{\prime \prime}, \quad a_{1}^{[3]}=a_{1}^{\prime \prime}$, and $a_{2}^{[3]}=a_{1} \wedge a_{1}^{\prime}$.

Lemma 1.5. Let $F$ be a free module over a commutative ring $R$. Let $a_{k}, b_{k}$, and $c_{k}$ be elements of $\Lambda^{k} F$ and $\alpha_{k}$ and $\beta_{k}$ be elements of $\bigwedge^{k} F^{*}$ for all integers $k$.

(a) If $\Delta\left(\alpha_{t}\right)=\sum_{j} \alpha_{1}^{[j]} \otimes \alpha_{t-1}^{[j]}$, then $\sum_{j} c_{t-2}\left(\alpha_{t-1}^{[j]}\right) \wedge \alpha_{1}^{[j]}=2(-1)^{t-1} c_{t-2}\left(\alpha_{t}\right)$.

(b) If $\Delta\left(\beta_{s}\right)=\sum \beta_{1}^{[i]} \otimes \beta_{s-1}^{[i]}$, then $\sum\left[\beta_{1}^{[i]}\left(a_{2}\right)\right]\left(\beta_{s-1}^{[i]}\right)=-2 a_{2}\left(\beta_{s}\right)$. 
Proof. Apply $F$ to each side of (a). If $b_{1}$ is a fixed, but arbitrary, element of $F$, then

$$
\begin{aligned}
& b_{1}\left(\sum_{j} c_{t-2}\left(\alpha_{t-1}^{[j]}\right) \wedge \alpha_{1}^{[j]}\right)=\sum_{j}\left(b_{1} \wedge c_{t-2}\right)\left(\alpha_{t-1}^{[j]}\right) \cdot \alpha_{1}^{[j]}-\sum_{j} b_{1}\left(\alpha_{1}^{[j]}\right) \cdot c_{t-2}\left(\alpha_{t-1}^{[j]}\right) \\
& =(-1)^{t-1}\left(b_{1} \wedge c_{t-2}\right)\left(\alpha_{t}\right)-c_{t-2}\left(b_{1}\left(\alpha_{t}\right)\right)=(-1)^{t-1} 2 b_{1}\left(c_{t-2}\left(\alpha_{t}\right)\right) .
\end{aligned}
$$

It suffices to prove (b) for $a_{2}=a_{1} \wedge a_{1}^{\prime}$. In this case,

$$
\begin{aligned}
\sum_{i}\left[\beta_{1}^{[i]}\left(a_{1} \wedge a_{1}^{\prime}\right)\right]\left(\beta_{s-1}^{[i]}\right) & =\sum_{i} a_{1}\left(\beta_{1}^{[i]}\right) \cdot a_{1}^{\prime}\left(\beta_{s-1}^{[i]}\right)-\sum_{i} a_{1}^{\prime}\left(\beta_{1}^{[i]}\right) \cdot a_{1}\left(\beta_{s-1}^{[i]}\right) \\
& =a_{1}^{\prime}\left(a_{1}\left(\beta_{s}\right)\right)-a_{1}\left(a_{1}^{\prime}\left(\beta_{s}\right)\right)=-2 a_{2}\left(\beta_{s}\right) .
\end{aligned}
$$

The following data is in effect throughout most of the paper.

Data 1.6. Let $R$ be a commutative noetherian ring, $F$ be a free module of rank $n \geq 2$ over $R, X: F \rightarrow F^{*}$ be an $R$-module homomorphism, and $u$ and $v$ be elements of $F$. Fix orientation elements $e_{n} \in \bigwedge^{n} F$ and $\varepsilon_{n} \in \bigwedge^{n} F^{*}$, which are compatible in the sense that $e_{n}\left(\varepsilon_{n}\right)=1$.

Note. We will always take $a_{i}$ and $b_{i}$ to be elements of $\bigwedge^{i} F$, and $\alpha_{i}$ and $\beta_{i}$ to be elements of $\bigwedge^{i} F^{*}$.

Remark 1.7. In the notation of Data 1.6, let $\beta_{1}^{[i]} \in \bigwedge^{1} F^{*}$ and $\alpha_{t} \in \bigwedge^{t} F^{*}$. In order to make sure that the reader understands our conventions, we give an expanded account of the symbol $\left[\beta_{1}^{[i]} \wedge\left(\bigwedge^{n-t} X^{*}\right)\left(\alpha_{t}\left[e_{n}\right]\right)\right]\left(e_{n}\right)$, which appears in Definition 2.1: $\alpha_{t}\left[e_{n}\right]$ is the element of $\bigwedge^{n-t} F$ which is given by the module action of $\bigwedge^{\bullet} F^{*}$ on $\bigwedge^{\bullet} F$; the map $\left(\bigwedge^{n-t} X^{*}\right)$ carries $\alpha_{t}\left[e_{n}\right]$ to an element of $\bigwedge^{n-t} F^{*}$; $\beta_{1}^{[i]} \wedge\left(\bigwedge^{n-t} X^{*}\right)\left(\alpha_{t}\left[e_{n}\right]\right)$ is an element of $\wedge^{n-t+1} F^{*}$; and the module action of $\bigwedge^{\bullet} F^{*}$ on $\bigwedge^{\bullet} F$ makes $\left[\beta_{1}^{[i]} \wedge\left(\bigwedge^{n-t} X^{*}\right)\left(\alpha_{t}\left[e_{n}\right]\right)\right]\left(e_{n}\right)$ an element of $\bigwedge^{t-1} F$.

The following convention and calculation provide the connection between the coordinate free complexes $\mathbb{F}$ and $\mathbb{M}$ of sections 2 and 3 and the coordinate dependent ideals $H(\mathbf{u}, \mathbf{X}, \mathbf{v})$ of Definition 1.1.

Convention 1.8. Whenever we convert $u, X$, and $v$ from Data 1.6 into matrices $\mathbf{u}, \mathbf{X}$, and $\mathbf{v}$, we consider a pair of bases $e_{1}^{[1]}, \ldots, e_{1}^{[n]}$ for $F$ and $\varepsilon_{1}^{[1]}, \ldots, \varepsilon_{1}^{[n]}$ for $F^{*}$ which satisfy $\varepsilon_{1}^{[i]}\left(e_{1}^{[j]}\right)=\delta_{i j}$ (the Kronecker delta), $e_{1}^{[1]} \wedge \ldots \wedge e_{1}^{[n]}=e_{n}$, and $\varepsilon_{1}^{[n]} \wedge \ldots \wedge \varepsilon_{1}^{[1]}=\varepsilon_{n}$. If $u=\sum_{i} u_{i} e_{1}^{[i]}, X\left(e_{1}^{[j]}\right)=\sum_{i} x_{i j} \varepsilon_{1}^{[i]}$, and $v=\sum_{i} v_{i} e_{1}^{[i]}$, then $\mathbf{u}=\left[u_{1}, \ldots, u_{n}\right], \mathbf{X}$ is the $n \times n$ matrix whose entry in row $i$ and column $j$ is $x_{i j}$ and $\mathbf{v}=\left[\begin{array}{c}v_{1} \\ \vdots \\ v_{n}\end{array}\right]$.

Lemma 1.9. Adopt Data 1.6. If $\mathbf{u}, \mathbf{X}$ and $\mathbf{v}$ are matrices which satisfy Convention 1.8 , then

$$
\left[\varepsilon^{[j]} \wedge\left(\Lambda^{n-1} X\right)\left(\varepsilon^{[i]}\left[e_{e}\right)\right]\left(e_{m}\right)=(-1)^{\frac{n(n-1)}{2}}(\operatorname{Adi} \mathbf{X})\right.
$$


Proof. Let $\mathbf{X}\left(r_{1}, \ldots, r_{s} ; c_{1}, \ldots, c_{s}\right)$ represent the determinant of the submatrix of $\mathbf{X}$ which consists of rows $r_{1}, \ldots, r_{s}$ and columns $c_{1}, \ldots, c_{s}$. The left side of the proposed identity is equal to

$$
\begin{aligned}
& (-1)^{i+1}\left[\varepsilon_{1}^{[j]} \wedge\left(\wedge^{n-1} X\right)\left(e_{1}^{[1]} \wedge \ldots \wedge \widehat{e_{1}^{[i]}} \wedge \ldots \wedge e_{1}^{[n]}\right)\right]\left(e_{n}\right) \\
& =(-1)^{i+1}\left[\varepsilon_{1}^{[j]} \wedge\left(\sum_{k} \mathbf{X}(1, \ldots, \widehat{k}, \ldots, n ; 1, \ldots, \widehat{i}, \ldots, n) \varepsilon_{1}^{[1]} \wedge \ldots \wedge \varepsilon_{1}^{[k]} \wedge \ldots \wedge \varepsilon_{1}^{[n]}\right)\right]\left(e_{n}\right) \\
& =(-1)^{i+j} \mathbf{X}(1, \ldots, \hat{j}, \ldots, n ; 1, \ldots, \widehat{i}, \ldots, n) \cdot\left(\varepsilon_{1}^{[1]} \wedge \ldots \wedge \varepsilon_{1}^{[n]}\right)\left(e_{n}\right) .
\end{aligned}
$$

The proof is complete since $\left(\varepsilon_{1}^{[1]} \wedge \ldots \wedge \varepsilon_{1}^{[n]}\right)\left(e_{n}\right)=(-1)^{\frac{n(n-1)}{2}}$, and

$$
(-1)^{i+j} \mathbf{X}(1, \ldots, \widehat{j}, \ldots, n ; 1, \ldots, \widehat{i}, \ldots, n)=(\operatorname{Adj} \mathbf{X})_{i j}
$$

Adopt Data 1.6. If $a_{1}$ and $b_{1}$ are elements of $F$, then the canonical identification of $F^{* *}$ with $F$ yields

$$
\left(X\left(b_{1}\right)\right)\left(a_{1}\right)=b_{1}\left(X^{*}\left(a_{1}\right)\right) .
$$

Furthermore, the canonical identification of $\left(\bigwedge^{j} F\right)^{*}$ with $\bigwedge^{j} F^{*}$ gives

$$
\left[\left(\bigwedge^{j} X\right)\left(b_{j}\right)\right]\left(a_{j}\right)=b_{j}\left(\left(\bigwedge^{j} X^{*}\right)\left(a_{j}\right)\right)
$$

for all $a_{j}$ and $b_{j}$ in $\bigwedge^{j} F$ and for all $j$. Formula (1.10) is the case " $i=0$ " of part (a) of the following result.

Observation 1.11. Adopt Data 1.6. Let $a_{k}$ and $b_{k}$ be elements of $\bigwedge^{k} F$ and $\alpha_{k}$ and $\beta_{k}$ be elements of $\bigwedge^{k} F^{*}$ for all integers $k$.

(a) $\left(\bigwedge^{i} X^{*}\right)\left[\left(\left(\bigwedge^{j} X\right)\left(b_{j}\right)\right)\left(a_{i+j}\right)\right]=b_{j}\left[\left(\bigwedge^{i+j} X^{*}\right)\left(a_{i+j}\right)\right]$.

(b) If $\Delta\left(a_{t}\right)=\sum_{j} a_{1}^{[j]} \otimes a_{t-1}^{[j]}$ and $\Delta\left(\beta_{s}\right)=\sum_{i} \beta_{1}^{[i]} \otimes \beta_{s-1}^{[i]}$, then

$$
\sum_{i} \beta_{1}^{[i]}\left(a_{t}\right) \otimes \beta_{s-1}^{[i]}=\sum_{j} a_{t-1}^{[j]} \otimes a_{1}^{[j]}\left(\beta_{s}\right) .
$$

(c) If $\Delta\left(\alpha_{t}\right)=\sum_{j} \alpha_{1}^{[j]} \otimes \alpha_{t-1}^{[j]}$ and $\Delta\left(\beta_{s}\right)=\sum_{i} \beta_{1}^{[i]} \otimes \beta_{s-1}^{[i]}$, then

$$
\sum_{i}\left(\left[\left(\bigwedge^{n-1} X\right)\left[\beta_{1}^{[i]}\left(e_{n}\right)\right]\right]\left(e_{n}\right)\right)\left(\alpha_{t}\right) \otimes \beta_{s-1}^{[i]}=\sum_{j} \alpha_{t-1}^{[j]} \otimes\left(\left[\left(\bigwedge^{n-1} X^{*}\right)\left[\alpha_{1}^{[j]}\left(e_{n}\right)\right]\right]\left(e_{n}\right)\right)\left(\beta_{s}\right) \text {. }
$$

Proof. We apply each side of (a) to the element $a_{i}$ of $\bigwedge^{i} F$. Notice that $\left(\left(\bigwedge^{j} X\right)\left(b_{j}\right)\right)\left(a_{i+j}\right)$ is in $\bigwedge^{i} F$. Use (1.10), together with the module actions of $\bigwedge^{\bullet} F$ and $\bigwedge^{\bullet} F^{*}$ on one another, to see that

$$
\begin{gathered}
\left(\left(\bigwedge^{i} X^{*}\right)\left[\left(\left(\bigwedge^{j} X\right)\left(b_{j}\right)\right)\left(a_{i+j}\right)\right]\right)\left(a_{i}\right)=\left[\left(\left(\bigwedge^{j} X\right)\left(b_{j}\right)\right)\left(a_{i+j}\right)\right]\left(\left(\bigwedge^{i} X\right)\left(a_{i}\right)\right) \\
=\left[\left(\bigwedge^{i} X\right)\left(a_{i}\right) \wedge\left(\bigwedge^{j} X\right)\left(b_{j}\right)\right]\left(a_{i+j}\right)=\left[\left(\bigwedge^{i+j} X\right)\left(a_{i} \wedge b_{j}\right)\right]\left(a_{i+j}\right) \\
=\left(a_{\cdot} \wedge h .\right)\left[\left(\bigwedge^{i+j} X^{*}\right)\left(a_{. .}\right)\right]=\left(h .\left[\left(\bigwedge^{i+j} X^{*}\right)(a . .)\right]\right)\left(a_{*}\right)
\end{gathered}
$$


Both expressions in (b) are equal to $\sum_{i} \sum_{j} \beta_{1}^{[i]}\left(a_{1}^{[j]}\right) \cdot a_{t-1}^{[j]} \otimes \beta_{s-1}^{[i]}$. Use the action of $F$ on $\bigwedge^{\bullet} F^{*}$, Proposition $1.2(\mathrm{~b})$, and (1.10) to see that the left side of (c) is equal to

$$
\begin{aligned}
& \sum_{i} \sum_{j}\left(\left[\left(\bigwedge^{n-1} X\right)\left[\beta_{1}^{[i]}\left(e_{n}\right)\right]\right]\left(e_{n}\right)\right)\left(\alpha_{1}^{[j]}\right) \cdot \alpha_{t-1}^{[j]} \otimes \beta_{s-1}^{[i]} \\
& =(-1)^{n-1} \sum_{i} \sum_{j}\left(\alpha_{1}^{[j]}\left[e_{n}\right]\right)\left[\left(\bigwedge^{n-1} X\right)\left[\beta_{1}^{[i]}\left(e_{n}\right)\right]\right] \cdot \alpha_{t-1}^{[j]} \otimes \beta_{s-1}^{[i]} \\
& =(-1)^{n-1} \sum_{i} \sum_{j}\left[\beta_{1}^{[i]}\left(e_{n}\right)\right]\left[\left(\bigwedge^{n-1} X^{*}\right)\left(\alpha_{1}^{[j]}\left(e_{n}\right)\right)\right] \cdot \alpha_{t-1}^{[j]} \otimes \beta_{s-1}^{[i]} \\
& =\sum_{j} \alpha_{t-1}^{[j]} \otimes \sum_{i}\left(\left[\left(\bigwedge^{n-1} X^{*}\right)\left(\alpha_{1}^{[j]}\left(e_{n}\right)\right)\right]\left(e_{n}\right)\right)\left(\beta_{1}^{[i]}\right) \cdot \beta_{s-1}^{[i]},
\end{aligned}
$$

which is equal to the right side of (c).

\section{The complex $\mathbb{F}$.}

The modules and maps which comprise the complex $\mathbb{F}$ are given in Definition 2.1. Recall the conventions of Remarks 1.4 and 1.7. If $t$ is an integer with $t \leq-1$ or $n+1 \leq t$, then $\bigwedge^{t} F=0$.

Definition 2.1. Adopt Data 1.6. The module $\mathbb{F}_{r}$ of $\mathbb{F}$ is defined to be

$$
\begin{aligned}
\mathbb{F}_{r}=\mathbb{F}_{r}(1) \oplus \mathbb{F}_{r}(2) \oplus \mathbb{F}_{r}(3) \oplus \mathbb{F}_{r}(4), & \text { where } \quad \mathbb{F}_{r}(i)=\sum_{t \in \mathbb{Z}} \mathbb{F}_{r}(i)^{(t)} \text { for } \\
\mathbb{F}_{r}(1)^{(t)}=\bigwedge^{t} F^{*} \otimes \bigwedge^{r+1-t} F^{*}, & \mathbb{F}_{r}(2)^{(t)}=\bigwedge^{t} F \otimes \bigwedge^{r-t} F^{*} \\
\mathbb{F}_{r}(3)^{(t)}=\bigwedge^{t} F \otimes \bigwedge^{r-t} F^{*}, \text { and } & \mathbb{F}_{r}(4)^{(t)}=\bigwedge^{t} F^{*} \otimes \bigwedge^{r-1-t} F^{*}
\end{aligned}
$$

The maps

$$
f_{r}(i)^{(t)}: \mathbb{F}_{r}(i)^{(t)} \rightarrow \mathbb{F}_{r-1}
$$

are given below.

If $s+t=r+1, \Delta\left(\alpha_{t}\right)=\sum_{j} \alpha_{1}^{[j]} \otimes \alpha_{t-1}^{[j]}$, and $\Delta\left(\beta_{s}\right)=\sum_{i} \beta_{1}^{[i]} \otimes \beta_{s-1}^{[i]}$, then

$$
f_{r}(1)^{(t)}\left(\alpha_{t} \otimes \beta_{s}\right)=\left\{\begin{array}{c}
\alpha_{t} \otimes v\left(\beta_{s}\right) \in \mathbb{F}_{r-1}(1)^{(t)} \\
+ \\
(-1)^{r} u\left(\alpha_{t}\right) \otimes \beta_{s} \in \mathbb{F}_{r-1}(1)^{(t-1)} \\
+ \\
\sum_{i}\left[\beta_{1}^{[i]} \wedge\left(\bigwedge^{n-t} X^{*}\right)\left(\alpha_{t}\left[e_{n}\right]\right)\right]\left(e_{n}\right) \otimes \beta_{s-1}^{[i]} \in \mathbb{F}_{r-1}(2)^{(t-1)} \\
+ \\
\sum_{j}\left[\alpha_{1}^{[j]} \wedge\left(\bigwedge^{n-s} X\right)\left(\beta_{s}\left[e_{n}\right]\right)\right]\left(e_{n}\right) \otimes \alpha_{t-1}^{[j]} \in \mathbb{F}_{r-1}(3)^{(s-1)} .
\end{array}\right.
$$

If $s+t=r$ and $\Delta\left(a_{t}\right)=\sum_{j} a_{1}^{[j]} \otimes a_{t-1}^{[j]}$, then

$$
f_{r}(2)^{(t)}\left(a_{t} \otimes \beta_{s}\right)=\left\{\begin{array}{c}
(-1)^{r}\left(\bigwedge^{t} X\right)\left(a_{t}\right) \otimes \beta_{s} \in \mathbb{F}_{r-1}(1)^{(t)} \\
+ \\
a_{t} \otimes v\left(\beta_{s}\right) \in \mathbb{F}_{r-1}(2)^{(t)} \\
+ \\
(-1)^{r+1}\left[X^{*}(u)\right]\left(a_{t}\right) \otimes \beta_{s} \in \mathbb{F}_{r-1}(2)^{(t-1)} \\
+{ }_{+} \\
\sum\left(\bigwedge^{t-1} X\right)\left(a_{t-1}^{[j]}\right) \otimes a_{1}^{[j]}\left(\beta_{s}\right) \in \mathbb{F}_{r-1}(4)^{(t-1)}
\end{array}\right.
$$


If $s+t=r$ and $\Delta\left(a_{t}\right)=\sum_{j} a_{1}^{[j]} \otimes a_{t-1}^{[j]}$, then

$$
f_{r}(3)^{(t)}\left(a_{t} \otimes \beta_{s}\right)=\left\{\begin{array}{c}
(-1)^{r+1} \beta_{s} \otimes\left(\bigwedge^{t} X^{*}\right)\left(a_{t}\right) \in \mathbb{F}_{r-1}(1)^{(s)} \\
+ \\
(-1)^{r+1} a_{t} \otimes u\left(\beta_{s}\right) \in \mathbb{F}_{r-1}(3)^{(t)} \\
+ \\
{[X(v)]\left(a_{t}\right) \otimes \beta_{s} \in \mathbb{F}_{r-1}(3)^{(t-1)}} \\
+ \\
-\sum_{j} a_{1}^{[j]}\left(\beta_{s}\right) \otimes\left(\bigwedge^{t-1} X^{*}\right)\left(a_{t-1}^{[j]}\right) \in \mathbb{F}_{r-1}(4)^{(s-1)} .
\end{array}\right.
$$

If $s+t=r-1$, then

$$
f_{r}(4)^{(t)}\left(\alpha_{t} \otimes \beta_{s}\right)=\left\{\begin{array}{c}
(-1)^{r}\left[\left(\bigwedge^{n-t} X^{*}\right)\left(\alpha_{t}\left[e_{n}\right]\right)\right]\left(e_{n}\right) \otimes \beta_{s} \in \mathbb{F}_{r-1}(2)^{(t)} \\
+ \\
(-1)^{r}\left[\left(\bigwedge^{n-s} X\right)\left(\beta_{s}\left[e_{n}\right]\right)\right]\left(e_{n}\right) \otimes \alpha_{t} \in \mathbb{F}_{r-1}(3)^{(s)} \\
+ \\
\alpha_{t} \otimes v\left(\beta_{s}\right) \in \mathbb{F}_{r-1}(4)^{(t)} \\
+ \\
(-1)^{r} u\left(\alpha_{t}\right) \otimes \beta_{s} \in \mathbb{F}_{r-1}(4)^{(t-1)} .
\end{array}\right.
$$

Proposition 2.2. The modules and maps of Definition 2.1 form a complex

$$
(\mathbb{F}, f): \quad 0 \rightarrow \mathbb{F}_{2 n+1} \rightarrow \ldots \rightarrow \mathbb{F}_{r} \stackrel{f_{r}}{\rightarrow} \mathbb{F}_{r-1} \rightarrow \ldots \rightarrow \mathbb{F}_{-1} \rightarrow 0
$$

Note. When we want to emphasize the data which was used to construct $(\mathbb{F}, f)$, we write $\mathbb{F}[u, X, v]$.

Proof. We prove that $f_{r-1} \circ f_{r}(\ell)^{(t)}=0$ for $1 \leq \ell \leq 4$. In each case we write

$f_{r-1} \circ f_{r}(\ell)^{(t)}\left(y_{t} \otimes \beta_{s}\right)=A+B+C+D, A=\sum_{k=1}^{4} A(k), B=\sum_{k=1}^{4} B(k), C=\sum_{k=1}^{4} C(k)$, and $D=\sum_{k=1}^{4} D(k)$,

where $y_{t}=\alpha_{t}$ if $\ell=1$ or 4 , and $y_{t}=a_{t}$ if $\ell=2$ or 3 .

The case $\ell=1$. Let $s+t=r+1, \Delta\left(\alpha_{t}\right)=\sum_{j} \alpha_{1}^{[j]} \otimes \alpha_{t-1}^{[j]}$, and $\Delta\left(\beta_{s}\right)=\sum_{i} \beta_{1}^{[i]} \otimes \beta_{s-1}^{[i]}$.

We have

$$
\begin{aligned}
& A=f_{r-1}(1)^{(t)}\left(\alpha_{t} \otimes v\left(\beta_{s}\right)\right), \\
& B=(-1)^{r} f_{r-1}(1)^{(t-1)}\left(u\left(\alpha_{t}\right) \otimes \beta_{s}\right), \\
& C=f_{r-1}(2)^{(t-1)}\left(\sum_{i}\left[\beta_{1}^{[i]} \wedge\left(\bigwedge^{n-t} X^{*}\right)\left(\alpha_{t}\left[e_{n}\right]\right)\right]\left(e_{n}\right) \otimes \beta_{s-1}^{[i]}\right), \\
& D=f_{r-1}(3)^{(s-1)}\left(\sum_{j}\left[\alpha_{1}^{[j]} \wedge\left(\bigwedge^{n-s} X\right)\left(\beta_{s}\left[e_{n}\right]\right)\right]\left(e_{n}\right) \otimes \alpha_{t-1}^{[j]}\right),
\end{aligned}
$$




$$
\begin{aligned}
& A(2)=(-1)^{r-1} u\left(\alpha_{t}\right) \otimes v\left(\beta_{s}\right) \in \mathbb{F}_{r-2}(1)^{(t-1)}, \\
& A(3)=-\sum_{i}\left[\beta_{1}^{[i]} \wedge\left(\bigwedge^{n-t} X^{*}\right)\left(\alpha_{t}\left[e_{n}\right]\right)\right]\left(e_{n}\right) \otimes v\left(\beta_{s-1}^{[i]}\right) \in \mathbb{F}_{r-2}(2)^{(t-1)}, \\
& A(4)=\sum_{j}\left[\alpha_{1}^{[j]} \wedge\left(\bigwedge^{n-s+1} X\right)\left(\left[v\left(\beta_{s}\right)\right]\left[e_{n}\right]\right)\right]\left(e_{n}\right) \otimes \alpha_{t-1}^{[j]} \in \mathbb{F}_{r-2}(3)^{(s-2)}, \\
& B(1)=(-1)^{r} u\left(\alpha_{t}\right) \otimes v\left(\beta_{s}\right) \in \mathbb{F}_{r-2}(1)^{(t-1)}, \\
& B(2)=-u\left(u\left(\alpha_{t}\right)\right) \otimes \beta_{s} \in \mathbb{F}_{r-2}(1)^{(t-2)}, \\
& B(3)=(-1)^{r} \sum_{i}\left[\beta_{1}^{[i]} \wedge\left(\bigwedge^{n-t+1} X^{*}\right)\left(\left[u\left(\alpha_{t}\right)\right]\left[e_{n}\right]\right)\right]\left(e_{n}\right) \otimes \beta_{s-1}^{[i]} \in \mathbb{F}_{r-2}(2)^{(t-2)}, \\
& B(4)=(-1)^{r+1} \sum_{j}\left[\alpha_{1}^{[j]} \wedge\left(\bigwedge^{n-s} X\right)\left(\beta_{s}\left[e_{n}\right]\right)\right]\left(e_{n}\right) \otimes u\left(\alpha_{t-1}^{[j]}\right) \in \mathbb{F}_{r-2}(3)^{(s-1)}, \\
& C(1)=(-1)^{r-1} \sum_{i}\left(\bigwedge^{t-1} X\right)\left(\left[\beta_{1}^{[i]} \wedge\left(\bigwedge^{n-t} X^{*}\right)\left(\alpha_{t}\left[e_{n}\right]\right)\right]\left(e_{n}\right)\right) \otimes \beta_{s-1}^{[i]} \in \mathbb{F}_{r-2}(1)^{(t-1)}, \\
& C(2)=\sum_{i}\left[\beta_{1}^{[i]} \wedge\left(\bigwedge^{n-t} X^{*}\right)\left(\alpha_{t}\left[e_{n}\right]\right)\right]\left(e_{n}\right) \otimes v\left(\beta_{s-1}^{[i]}\right) \in \mathbb{F}_{r-2}(2)^{(t-1)}, \\
& C(3)=(-1)^{r} \sum_{i}\left[X^{*}(u)\right]\left(\left[\beta_{1}^{[i]} \wedge\left(\bigwedge^{n-t} X^{*}\right)\left(\alpha_{t}\left[e_{n}\right]\right)\right]\left(e_{n}\right)\right) \otimes \beta_{s-1}^{[i]} \in \mathbb{F}_{r-2}(2)^{(t-2)}, \\
& C(4)=\text { the } \mathbb{F}_{r-2}(4)^{(t-2)}-\text { component of } f_{r-1}(2)^{(t-1)}\left(\sum_{i}\left[\beta_{1}^{[i]} \wedge\left(\bigwedge^{n-t} X^{*}\right)\left(\alpha_{t}\left[e_{n}\right]\right)\right]\left(e_{n}\right) \otimes \beta_{s-1}^{[i]}\right) \text {, } \\
& D(1)=(-1)^{r} \sum_{j} \alpha_{t-1}^{[j]} \otimes\left(\bigwedge^{s-1} X^{*}\right)\left(\left[\alpha_{1}^{[j]} \wedge\left(\bigwedge^{n-s} X\right)\left(\beta_{s}\left[e_{n}\right]\right)\right]\left(e_{n}\right)\right) \in \mathbb{F}_{r-2}(1)^{(t-1)} \\
& D(2)=(-1)^{r} \sum_{j}\left[\alpha_{1}^{[j]} \wedge\left(\bigwedge^{n-s} X\right)\left(\beta_{s}\left[e_{n}\right]\right)\right]\left(e_{n}\right) \otimes u\left(\alpha_{t-1}^{[j]}\right) \in \mathbb{F}_{r-2}(3)^{(s-1)}, \\
& D(3)=\sum_{j}[X(v)]\left(\left[\alpha_{1}^{[j]} \wedge\left(\bigwedge^{n-s} X\right)\left(\beta_{s}\left[e_{n}\right]\right)\right]\left(e_{n}\right)\right) \otimes \alpha_{t-1}^{[j]} \in \mathbb{F}_{r-2}(3)^{(s-2)}, \quad \text { and } \\
& D(4)=\text { the } \mathbb{F}_{r-2}(4)^{(t-2)} \text {-component of } f_{r-1}(3)^{(s-1)}\left(\sum_{j}\left[\alpha_{1}^{[j]} \wedge\left(\bigwedge^{n-s} X\right)\left(\beta_{s}\left[e_{n}\right]\right)\right]\left(e_{n}\right) \otimes \alpha_{t-1}^{[j]}\right) .
\end{aligned}
$$

Observe that

$$
0=A(1)=B(2)=A(2)+B(1)=A(3)+C(2)=A(4)+D(3)=B(3)+C(3)=B(4)+D(2) .
$$

Use the module action of $\bigwedge^{\bullet} F^{*}$ on $\bigwedge^{\bullet} F$, together with Observation 1.11 (a) and Proposition $1.2(\mathrm{~b})$, to see that

$$
\begin{aligned}
C(1) & =(-1)^{n-t}(-1)^{r-1} \sum_{i}\left(\alpha_{t}\left[e_{n}\right]\right)\left(\left(\bigwedge^{n-1} X\right)\left(\beta_{1}^{[i]}\left[e_{n}\right]\right)\right) \otimes \beta_{s-1}^{[i]} \\
& =(-1)^{r-1} \sum_{i}\left(\left[\left(\bigwedge^{n-1} X\right)\left(\beta_{1}^{[i]}\left[e_{n}\right]\right)\right]\left(e_{n}\right)\right)\left(\alpha_{t}\right) \otimes \beta_{s-1}^{[i]}, \text { and } \\
D(1) & =(-1)^{n-s}(-1)^{r} \sum \alpha_{t-1}^{[j]} \otimes\left(\beta_{s}\left[e_{n}\right]\right)\left(\left(\bigwedge^{n-1} X^{*}\right)\left(\alpha_{1}^{[j]}\left[e_{n}\right]\right)\right)
\end{aligned}
$$




$$
=(-1)^{r} \sum_{j} \alpha_{t-1}^{[j]} \otimes\left(\left[\left(\bigwedge^{n-1} X^{*}\right)\left(\alpha_{1}^{[j]}\left[e_{n}\right]\right)\right]\left(e_{n}\right)\right)\left(\beta_{s}\right) .
$$

Apply Observation 1.11 (c) to see that $C(1)+D(1)=0$.

We prove $C(4)+D(4)=0$ by showing that $\left(c_{t-2} \otimes 1\right) *(C(4)+D(4))=0$ for all $c_{t-2} \in \bigwedge^{t-2} F$, where

$$
\left(c_{t-2} \otimes 1\right) *\left(\alpha_{t-2} \otimes \beta\right)=c_{t-2}\left(\alpha_{t-2}\right) \cdot \beta \in \bigwedge^{\bullet} F^{*}
$$

for $\alpha_{t-2} \otimes \beta \in \bigwedge^{t-2} F^{*} \otimes \bigwedge^{\bullet} F^{*}$. Let $c_{t-2}$ be a fixed, but arbitrary, element of $\bigwedge^{t-2} F$. Observe that

$$
\begin{aligned}
& \left(c_{t-2} \otimes 1\right) *\left(\text { the } \mathbb{F}_{r-2}(4)^{(t-2)}-\text { component of } f_{r-1}(2)^{(t-1)}\left(a_{t-1} \otimes \beta_{s-1}\right)\right) \\
& =\sum_{j} c_{t-2}\left(\left(\bigwedge^{t-2} X\right)\left(a_{t-2}^{[j]}\right)\right) \cdot a_{1}^{[j]}\left(\beta_{s-1}\right)=\sum_{j}\left(\left(\bigwedge^{t-2} X^{*}\right)\left(c_{t-2}\right)\right)\left(a_{t-2}^{[j]}\right) \cdot a_{1}^{[j]}\left(\beta_{s-1}\right) \\
& =(-1)^{t}\left[\left[\left(\bigwedge^{t-2} X^{*}\right)\left(c_{t-2}\right)\right]\left(a_{t-1}\right)\right]\left(\beta_{s-1}\right),
\end{aligned}
$$

where $\Delta\left(a_{t-1}\right)=\sum_{j} a_{1}^{[j]} \otimes a_{t-2}^{[j]}$. It follows that

$$
\begin{aligned}
\left(c_{t-2} \otimes 1\right) * C(4) & =(-1)^{t} \sum_{i}\left[\left[\left(\bigwedge^{t-2} X^{*}\right)\left(c_{t-2}\right)\right]\left(\left[\beta_{1}^{[i]} \wedge\left(\bigwedge^{n-t} X^{*}\right)\left(\alpha_{t}\left(e_{n}\right)\right)\right]\left(e_{n}\right)\right)\right]\left(\beta_{s-1}^{[i]}\right) \\
& =\sum_{i}\left(\left[\beta_{1}^{[i]} \wedge\left(\bigwedge^{n-2} X^{*}\right)\left(c_{t-2} \wedge \alpha_{t}\left(e_{n}\right)\right)\right]\left(e_{n}\right)\right)\left(\beta_{s-1}^{[i]}\right) .
\end{aligned}
$$

Use Lemma $1.5(\mathrm{~b})$ and Corollary 1.3 to see that

$$
\begin{aligned}
\left(c_{t-2} \otimes 1\right) * C(4) & =-2\left(\left[\left(\bigwedge^{n-2} X^{*}\right)\left(c_{t-2} \wedge \alpha_{t}\left(e_{n}\right)\right)\right]\left(e_{n}\right)\right)\left(\beta_{s}\right) \\
& =-2\left(\left[\left(\bigwedge^{n-2} X^{*}\right)\left(\left[c_{t-2}\left(\alpha_{t}\right)\right]\left(e_{n}\right)\right)\right]\left(e_{n}\right)\right)\left(\beta_{s}\right) .
\end{aligned}
$$

Observe, also, that

$$
\begin{aligned}
& \left(c_{t-2} \otimes 1\right) *\left(\text { the } \mathbb{F}_{r-2}(4)^{(t-2)}-\text { component of } f_{r-1}(3)^{(s-1)}\left(a_{s-1} \otimes \beta_{t-1}\right)\right) \\
& =-\sum_{j} c_{t-2}\left(a_{1}^{[j]}\left(\beta_{t-1}\right)\right) \cdot\left(\bigwedge^{s-2} X^{*}\right)\left(a_{s-2}^{[j]}\right)=(-1)^{t-1}\left(\bigwedge^{s-2} X^{*}\right)\left(\sum_{j}\left[c_{t-2}\left(\beta_{t-1}\right)\right]\left(a_{1}^{[j]}\right) \cdot a_{s-2}^{[j]}\right) \\
& =(-1)^{t-1}\left(\bigwedge^{s-2} X^{*}\right)\left(\left[c_{t-2}\left(\beta_{t-1}\right)\right]\left(a_{s-1}\right)\right)
\end{aligned}
$$

where $\Delta\left(a_{s-1}\right)=\sum_{j} a_{1}^{[j]} \otimes a_{s-2}^{[j]}$. It follows that $\left(c_{t-2} \otimes 1\right) * D(4)$ is equal to

$$
(-1)^{t-1}\left(\bigwedge^{s-2} X^{*}\right)\left(\sum\left[c_{t-2}\left(\alpha_{t-1}^{[j]}\right)\right]\left(\left[\alpha_{1}^{[j]} \wedge\left(\bigwedge^{n-s} X\right)\left(\beta_{s}\left(e_{n}\right)\right)\right]\left(e_{n}\right)\right)\right)
$$


Apply Lemma 1.5 (a), Observation 1.11 (a), and Proposition 1.2 (b) to see that

$$
\begin{aligned}
& \left(c_{t-2} \otimes 1\right) * D(4)=2\left(\bigwedge^{s-2} X^{*}\right)\left(\left[c_{t-2}\left(\alpha_{t}\right) \wedge\left(\bigwedge^{n-s} X\right)\left(\beta_{s}\left[e_{n}\right]\right)\right]\left(e_{n}\right)\right) \\
& =2\left(\beta_{s}\left[e_{n}\right]\right)\left[\left(\bigwedge^{n-2} X^{*}\right)\left(\left[c_{t-2}\left(\alpha_{t}\right)\right]\left(e_{n}\right)\right)\right] \\
& =2\left[\left[\left(\bigwedge^{n-2} X^{*}\right)\left(\left[c_{t-2}\left(\alpha_{t}\right)\right]\left(e_{n}\right)\right)\right]\left(e_{n}\right)\right]\left(\beta_{s}\right)=-\left(c_{t-2} \otimes 1\right) * C(4) ;
\end{aligned}
$$

therefore, $C(4)+D(4)=0$ and $f_{r-1} \circ f_{r}(1)^{(t)}\left(\alpha_{t} \otimes \beta_{s}\right)=0$.

The case $\ell=2$. Let $s+t=r, \Delta\left(a_{t}\right)=\sum_{j} a_{1}^{[j]} \otimes a_{t-1}^{[j]}$, and $\Delta\left(\beta_{s}\right)=\sum_{i} \beta_{1}^{[i]} \otimes \beta_{s-1}^{[i]}$.

We have

$$
\begin{aligned}
& A=(-1)^{r} f_{r-1}(1)^{(t)}\left(\left(\bigwedge^{t} X\right)\left(a_{t}\right) \otimes \beta_{s}\right), \\
& B=f_{r-1}(2)^{(t)}\left(a_{t} \otimes v\left(\beta_{s}\right)\right), \\
& C=(-1)^{r+1} f_{r-1}(2)^{(t-1)}\left(\left[X^{*}(u)\right]\left(a_{t}\right) \otimes \beta_{s}\right), \\
& D=f_{r-1}(4)^{(t-1)}\left(\sum_{j}\left(\bigwedge^{t-1} X\right)\left(a_{t-1}^{[j]}\right) \otimes a_{1}^{[j]}\left(\beta_{s}\right)\right),
\end{aligned}
$$

$$
\begin{aligned}
& A(1)=(-1)^{r}\left(\bigwedge^{t} X\right)\left(a_{t}\right) \otimes v\left(\beta_{s}\right) \in \mathbb{F}_{r-2}(1)^{(t)}, \\
& A(2)=-u\left(\left(\bigwedge^{t} X\right)\left(a_{t}\right)\right) \otimes \beta_{s} \in \mathbb{F}_{r-2}(1)^{(t-1)}, \\
& A(3)=(-1)^{r} \sum_{i}\left[\beta_{1}^{[i]} \wedge\left(\bigwedge^{n-t} X^{*}\right)\left(\left[\left(\bigwedge^{t} X\right)\left(a_{t}\right)\right]\left[e_{n}\right]\right)\right]\left(e_{n}\right) \otimes \beta_{s-1}^{[i]} \in \mathbb{F}_{r-2}(2)^{(t-1)} \\
& A(4)=(-1)^{r} \sum_{j}\left[X\left(a_{1}^{[j]}\right) \wedge\left(\bigwedge^{n-s} X\right)\left(\beta_{s}\left[e_{n}\right]\right)\right]\left(e_{n}\right) \otimes\left(\bigwedge^{t-1} X\right)\left(a_{t-1}^{[j]}\right) \in \mathbb{F}_{r-2}(3)^{(s-1)}, \\
& B(1)=(-1)^{r-1}\left(\bigwedge^{t} X\right)\left(a_{t}\right) \otimes v\left(\beta_{s}\right) \in \mathbb{F}_{r-2}(1)^{(t)}, \\
& B(2)=a_{t} \otimes v\left[v\left(\beta_{s}\right)\right] \in \mathbb{F}_{r-2}(2)^{(t)}, \\
& B(3)=(-1)^{r}\left[X^{*}(u)\right]\left(a_{t}\right) \otimes v\left(\beta_{s}\right) \in \mathbb{F}_{r-2}(2)^{(t-1)}, \\
& B(4)=\sum_{j}\left(\bigwedge^{t-1} X\right)\left(a_{t-1}^{[j]}\right) \otimes a_{1}^{[j]}\left(v\left[\beta_{s}\right]\right) \in \mathbb{F}_{r-2}(4)^{(t-1)}, \\
& C(1)=\left(\bigwedge^{t-1} X\right)\left(\left[X^{*}(u)\right]\left(a_{t}\right)\right) \otimes \beta_{s} \in \mathbb{F}_{r-2}(1)^{(t-1)}, \\
& C(2)=(-1)^{r+1}\left[X^{*}(u)\right]\left(a_{t}\right) \otimes v\left(\beta_{s}\right) \in \mathbb{F}_{r-2}(2)^{(t-1)}, \\
& C(3)=-\left[X^{*}(u)\right]\left(\left[X^{*}(u)\right]\left(a_{t}\right)\right) \otimes \beta_{s} \in \mathbb{F}_{r-2}(2)^{(t-2)}, \\
& D(1)=(-1)^{r-1} \sum_{j}\left[\left(\bigwedge^{n-t+1} X^{*}\right)\left[\left(\left(\bigwedge^{t-1} X\right)\left(a_{t-1}^{[j]}\right)\right)\left(e_{n}\right)\right]\right]\left(e_{n}\right) \otimes a_{1}^{[j]}\left(\beta_{s}\right) \in \mathbb{F}_{r-2}(2)^{(t-1)} \\
& D(2)=(-1)^{r-1} \sum_{t}\left[\left(\bigwedge^{n-s+1} X\right)\left[\left(a_{1}^{[j]}\left(\beta_{s}\right)\right)\left(e_{n}\right)\right]\right]\left(e_{n}\right) \otimes\left(\bigwedge^{t-1} X\right)\left(a_{t-1}^{[j]}\right) \in \mathbb{F}_{r-2}(3)^{(s-1)}
\end{aligned}
$$




$$
\begin{aligned}
& D(3)=\sum_{j}\left(\bigwedge^{t-1} X\right)\left(a_{t-1}^{[j]}\right) \otimes v\left(a_{1}^{[j]}\left(\beta_{s}\right)\right) \in \mathbb{F}_{r-2}(4)^{(t-1)}, \quad \text { and } \\
& D(4)=(-1)^{r-1} \sum_{j} u\left(\left(\bigwedge^{t-1} X\right)\left(a_{t-1}^{[j]}\right)\right) \otimes a_{1}^{[j]}\left(\beta_{s}\right) \in \mathbb{F}_{r-2}(4)^{(t-2)} .
\end{aligned}
$$

Observe that

$0=B(2)=C(3)=A(1)+B(1)=A(2)+C(1)=A(4)+D(2)=B(3)+C(2)=B(4)+D(3)=C(4)+D(4)$.

Furthermore, Observation $1.11(\mathrm{a})$ and (b), Proposition 1.2(b), and Corollary 1.3 give

$$
\begin{aligned}
& A(3)=(-1)^{r}\left[\left(\bigwedge^{n} X\right)\left(e_{n}\right)\right]\left(e_{n}\right) \cdot \sum_{i} \beta_{1}^{[i]}\left(a_{t}\right) \otimes \beta_{s-1}^{[i]} \\
& =(-1)^{r}\left[\left(\bigwedge^{n} X\right)\left(e_{n}\right)\right]\left(e_{n}\right) \cdot \sum_{j} a_{t-1}^{[j]} \otimes a_{1}^{[j]}\left(\beta_{s}\right)=-D(1) ;
\end{aligned}
$$

thus, $f_{r-1} \circ f_{r}(2)^{(t)}\left(a_{t} \otimes \beta_{s}\right)=0$.

The case $\ell=3$. Let $s+t=r, \Delta\left(a_{t}\right)=\sum_{j} a_{1}^{[j]} \otimes a_{t-1}^{[j]}$, and $\Delta\left(\beta_{s}\right)=\sum_{i} \beta_{1}^{[i]} \otimes \beta_{s-1}^{[i]}$.

We have

$$
\begin{aligned}
& A=(-1)^{r+1} f_{r-1}(1)^{(s)}\left(\beta_{s} \otimes\left(\bigwedge^{t} X^{*}\right)\left(a_{t}\right)\right), \\
& B=(-1)^{r+1} f_{r-1}(3)^{(t)}\left(a_{t} \otimes u\left(\beta_{s}\right)\right), \\
& C=f_{r-1}(3)^{(t-1)}\left([X(v)]\left(a_{t}\right) \otimes \beta_{s}\right), \\
& D=-f_{r-1}(4)^{(s-1)}\left(\sum_{j} a_{1}^{[j]}\left(\beta_{s}\right) \otimes\left(\bigwedge^{t-1} X^{*}\right)\left(a_{t-1}^{[j]}\right)\right),
\end{aligned}
$$

$$
\begin{aligned}
& A(1)=(-1)^{r+1} \beta_{s} \otimes v\left(\left(\bigwedge^{t} X^{*}\right)\left(a_{t}\right)\right) \in \mathbb{F}_{r-2}(1)^{(s)}, \\
& A(2)=u\left(\beta_{s}\right) \otimes\left(\bigwedge^{t} X^{*}\right)\left(a_{t}\right) \in \mathbb{F}_{r-2}(1)^{(s-1)}, \\
& A(3)=(-1)^{r+1} \sum_{j}\left[X^{*}\left(a_{1}^{[j]}\right) \wedge\left(\bigwedge^{n-s} X^{*}\right)\left(\beta_{s}\left[e_{n}\right]\right)\right]\left(e_{n}\right) \otimes\left(\bigwedge^{t-1} X^{*}\right)\left(a_{t-1}^{[j]}\right) \in \mathbb{F}_{r-2}(2)^{(s-1)}, \\
& \left.A(4)=(-1)^{r+1} \sum_{i}\left[\beta_{1}^{[i]} \wedge\left(\bigwedge^{n-t} X\right)\left[\left[\bigwedge^{t} X^{*}\right)\left(a_{t}\right)\right]\left[e_{n}\right]\right]\right]\left(e_{n}\right) \otimes \beta_{s-1}^{[i]} \in \mathbb{F}_{r-2}(3)^{(t-1)}, \\
& B(1)=-u\left(\beta_{s}\right) \otimes\left(\bigwedge^{t} X^{*}\right)\left(a_{t}\right) \in \mathbb{F}_{r-2}(1)^{(s-1)}, \\
& B(2)=-a_{t} \otimes u\left(u\left(\beta_{s}\right)\right) \in \mathbb{F}_{r-2}(3)^{(t)}, \\
& B(3)=(-1)^{r+1}[X(v)]\left(a_{t}\right) \otimes u\left(\beta_{s}\right) \in \mathbb{F}_{r-2}(3)^{(t-1)}, \\
& B(4)=(-1)^{r} \sum_{j} a_{1}^{[j]}\left(u\left[\beta_{s}\right]\right) \otimes\left(\bigwedge^{t-1} X^{*}\right)\left(a_{t-1}^{[j]}\right) \in \mathbb{F}_{r-2}(4)^{(s-2)}, \\
& C(1)=(-1)^{r} \beta_{s} \otimes\left(\bigwedge^{t-1} X^{*}\right)\left([X(v)]\left(a_{t}\right)\right) \in \mathbb{F}_{r-2}(1)^{(s)}, \\
& C(2)=(-1)^{r}[X(v)]\left(a_{t}\right) \otimes u\left(\beta_{s}\right) \in \mathbb{F}_{r-2}(3)^{(t-1)},
\end{aligned}
$$




$$
\begin{aligned}
& C(4)=\sum_{j} a_{1}^{[j]}\left(\beta_{s}\right) \otimes v\left[\left(\bigwedge^{t-1} X^{*}\right)\left(a_{t-1}^{[j]}\right)\right] \in \mathbb{F}_{r-2}(4)^{(s-1)}, \\
& D(1)=(-1)^{r} \sum_{j}\left[\left(\bigwedge^{n-s+1} X^{*}\right)\left[\left(a_{1}^{[j]}\left(\beta_{s}\right)\right)\left(e_{n}\right)\right]\right]\left(e_{n}\right) \otimes\left(\bigwedge^{t-1} X^{*}\right)\left(a_{t-1}^{[j]}\right) \in \mathbb{F}_{r-2}(2)^{(s-1)}, \\
& D(2)=(-1)^{r} \sum_{j}\left[\left(\bigwedge^{n-t+1} X\right)\left[\left(\left(\bigwedge^{t-1} X^{*}\right)\left(a_{t-1}^{[j]}\right)\right)\left(e_{n}\right)\right]\right]\left(e_{n}\right) \otimes a_{1}^{[j]}\left(\beta_{s}\right) \in \mathbb{F}_{r-2}(3)^{(t-1)}, \\
& D(3)=-\sum_{j} a_{1}^{[j]}\left(\beta_{s}\right) \otimes v\left[\left(\bigwedge^{t-1} X^{*}\right)\left(a_{t-1}^{[j]}\right)\right] \in \mathbb{F}_{r-2}(4)^{(s-1)}, \quad \text { and } \\
& D(4)=(-1)^{r} \sum_{j} u\left[a_{1}^{[j]}\left(\beta_{s}\right)\right] \otimes\left(\bigwedge^{t-1} X^{*}\right)\left(a_{t-1}^{[j]}\right) \in \mathbb{F}_{r-2}(4)^{(s-2)} .
\end{aligned}
$$

\section{Observe that}

$0=B(2)=C(3)=A(1)+C(1)=A(2)+B(1)=A(3)+D(1)=B(3)+C(2)=B(4)+D(4)=C(4)+D(3)$.

The argument of $(2.4)$ gives $A(4)+D(2)=0$; and therefore, $f_{r-1} \circ f_{r}(3)^{(t)}\left(a_{t} \otimes \beta_{s}\right)=$ 0 .

The case $\ell=4$. Let $s+t=r-1$. We have

$$
\begin{aligned}
& A=(-1)^{r} f_{r-1}(2)^{(t)}\left(\left[\left(\bigwedge^{n-t} X^{*}\right)\left(\alpha_{t}\left[e_{n}\right]\right)\right]\left(e_{n}\right) \otimes \beta_{s}\right), \\
& B=(-1)^{r} f_{r-1}(3)^{(s)}\left(\left[\left(\bigwedge^{n-s} X\right)\left(\beta_{s}\left[e_{n}\right]\right)\right]\left(e_{n}\right) \otimes \alpha_{t}\right), \\
& C=f_{r-1}(4)^{(t)}\left(\alpha_{t} \otimes v\left(\beta_{s}\right)\right) \\
& D=(-1)^{r} f_{r-1}(4)^{(t-1)}\left(u\left(\alpha_{t}\right) \otimes \beta_{s}\right)
\end{aligned}
$$

$$
\begin{aligned}
& A(1)=-\left(\bigwedge^{t} X\right)\left(\left[\left(\bigwedge^{n-t} X^{*}\right)\left(\alpha_{t}\left[e_{n}\right]\right)\right]\left(e_{n}\right)\right) \otimes \beta_{s} \in \mathbb{F}_{r-2}(1)^{(t)}, \\
& A(2)=(-1)^{r}\left[\left(\bigwedge^{n-t} X^{*}\right)\left(\alpha_{t}\left[e_{n}\right]\right)\right]\left(e_{n}\right) \otimes v\left(\beta_{s}\right) \in \mathbb{F}_{r-2}(2)^{(t)}, \\
& A(3)=\left[X^{*}(u)\right]\left(\left[\left(\bigwedge^{n-t} X^{*}\right)\left(\alpha_{t}\left[e_{n}\right]\right)\right]\left(e_{n}\right)\right) \otimes \beta_{s} \in \mathbb{F}_{r-2}(2)^{(t-1)}, \\
& A(4)=\text { the } \mathbb{F}_{r-2}(4)^{(t-1)}-\text { component of }(-1)^{r} f_{r-1}(2)^{(t)}\left(\left[\left(\bigwedge^{n-t} X^{*}\right)\left(\alpha_{t}\left[e_{n}\right]\right)\right]\left(e_{n}\right) \otimes \beta_{s}\right), \\
& B(1)=\alpha_{t} \otimes\left(\bigwedge^{s} X^{*}\right)\left(\left[\left(\bigwedge^{n-s} X\right)\left(\beta_{s}\left[e_{n}\right]\right)\right]\left(e_{n}\right)\right) \in \mathbb{F}_{r-2}(1)^{(t)}, \\
& B(2)=\left[\left(\bigwedge^{n-s} X\right)\left(\beta_{s}\left[e_{n}\right]\right)\right]\left(e_{n}\right) \otimes u\left(\alpha_{t}\right) \in \mathbb{F}_{r-2}(3)^{(s)}, \\
& B(3)=(-1)^{r}[X(v)]\left(\left[\left(\bigwedge^{n-s} X\right)\left(\beta_{s}\left[e_{n}\right]\right)\right]\left(e_{n}\right)\right) \otimes \alpha_{t} \in \mathbb{F}_{r-2}(3)^{(s-1)}, \\
& B(4)=\operatorname{the} \mathbb{F}_{r-2}(4)^{(t-1)}-\operatorname{component} \text { of }(-1)^{r} f_{r-1}(3)^{(s)}\left(\left[\left(\bigwedge^{n-s} X\right)\left(\beta_{s}\left[e_{n}\right]\right)\right]\left(e_{n}\right) \otimes \alpha_{t}\right), \\
& C(1)=(-1)^{r-1}\left[\left(\bigwedge^{n-t} X^{*}\right)\left(\alpha_{t}\left[e_{n}\right]\right)\right]\left(e_{n}\right) \otimes v\left(\beta_{s}\right) \in \mathbb{F}_{r-2}(2)^{(t)}, \\
& C(2)=(-1)^{r-1}\left[\left(\bigwedge^{n-s+1} X\right)\left(\left(v\left[\beta_{s}\right]\right)\left[e_{n}\right]\right)\right]\left(e_{n}\right) \otimes \alpha_{t} \in \mathbb{F}_{r-2}(3)^{(s-1)}, \\
& C(3)=\alpha_{t} \otimes v\left(v\left(\beta_{s}\right)\right) \in \mathbb{F}_{r-2}(4)^{(t)}, \\
& C(4)=(-1)^{r-1} u\left(\alpha_{t}\right) \otimes v\left(\beta_{s}\right) \in \mathbb{F}_{r-2}(4)^{(t-1)}, \\
& D(1)=-\left[\left(\bigwedge^{n-t+1} X^{*}\right)\left(\left[u\left(\alpha_{t}\right)\right]\left[e_{n}\right]\right)\right]\left(e_{n}\right) \otimes \beta_{s} \in \mathbb{F}_{r-2}(2)^{(t-1)}, \\
& D(2)=-\left[\left(\bigwedge^{n-s} X\right)\left(\beta_{s}\left[e_{n}\right]\right)\right]\left(e_{n}\right) \otimes u\left(\alpha_{t}\right) \in \mathbb{F}_{r-2}(3)^{(s)},
\end{aligned}
$$




$$
D(4)=-u\left(u\left(\alpha_{t}\right)\right) \otimes \beta_{s} \in \mathbb{F}_{r-2}(4)^{(t-2)} .
$$

Observe that

$0=C(3)=D(4)=A(1)+B(1)=A(2)+C(1)=A(3)+D(1)=B(2)+D(2)=B(3)+C(2)=C(4)+D(3)$.

Let $c_{t-1}$ be a fixed, but arbitrary, element of $\bigwedge^{t-1} F$. Employ the trick of (2.3). We see that

$$
\begin{aligned}
& \left(c_{t-1} \otimes 1\right) *\left(\text { the } \mathbb{F}_{r-2}(4)^{(t-1)} \text {-component of } f_{r-1}(2)^{(t)}\left(a_{t} \otimes \beta_{s}\right)\right) \\
& =\sum_{j} c_{t-1}\left(\left(\bigwedge^{t-1} X\right)\left(a_{t-1}^{[j]}\right)\right) \cdot a_{1}^{[j]}\left(\beta_{s}\right)=\left(\sum_{j}\left[\left(\bigwedge^{t-1} X^{*}\right)\left(c_{t-1}\right)\right]\left(a_{t-1}^{[j]}\right) \cdot a_{1}^{[j]}\right)\left(\beta_{s}\right) \\
& =(-1)^{t-1}\left(\left[\left(\bigwedge^{t-1} X^{*}\right)\left(c_{t-1}\right)\right]\left(a_{t}\right)\right)\left(\beta_{s}\right),
\end{aligned}
$$

where $\Delta\left(a_{t}\right)=\sum_{j} a_{1}^{[j]} \otimes a_{t-1}^{[j]} ;$ and therefore, it follows that $\left(c_{t-1} \otimes 1\right) * A(4)$ is equal to

$$
\begin{aligned}
& (-1)^{t-1+r}\left(\left[\left(\bigwedge^{t-1} X^{*}\right)\left(c_{t-1}\right)\right]\left(\left[\left(\bigwedge^{n-t} X^{*}\right)\left(\alpha_{t}\left[e_{n}\right]\right)\right]\left(e_{n}\right)\right)\right)\left(\beta_{s}\right) \\
& =(-1)^{t-1+r}\left(\left[\left(\bigwedge^{n-1} X^{*}\right)\left(\left[c_{t-1}\left(\alpha_{t}\right)\right]\left[e_{n}\right]\right)\right]\left(e_{n}\right)\right)\left(\beta_{s}\right) \\
& =(-1)^{n-s}(-1)^{t-1+r}\left[\beta_{s}\left(e_{n}\right)\right]\left[\left(\bigwedge^{n-1} X^{*}\right)\left(\left[c_{t-1}\left(\alpha_{t}\right)\right]\left(e_{n}\right)\right)\right] .
\end{aligned}
$$

We also see that $\left(c_{t-1} \otimes 1\right) *\left(\right.$ the $\mathbb{F}_{r-2}(4)^{(t-1)}$ - component of $\left.f_{r-1}(3)^{(s)}\left(b_{s} \otimes \alpha_{t}\right)\right)$ is equal to

$$
\begin{aligned}
-\sum_{i} c_{t-1}\left(b_{1}^{[i]}\left(\alpha_{t}\right)\right) \cdot\left(\bigwedge^{s-1} X^{*}\right)\left(b_{s-1}^{[i]}\right) & =(-1)^{t}\left(\bigwedge^{s-1} X^{*}\right)\left[\sum_{i}\left(c_{t-1}\left(\alpha_{t}\right)\right)\left(b_{1}^{[i]}\right) \cdot b_{s-1}^{[i]}\right] \\
& =(-1)^{t}\left(\bigwedge^{s-1} X^{*}\right)\left[\left(c_{t-1}\left(\alpha_{t}\right)\right)\left(b_{s}\right)\right]
\end{aligned}
$$

where $\Delta\left(b_{s}\right)=\sum_{i} b_{1}^{[i]} \otimes b_{s-1}^{[i]}$; therefore, $\left(c_{t-1} \otimes 1\right) * B(4)$ is equal to

$$
\begin{aligned}
& (-1)^{t+r}\left(\bigwedge^{s-1} X^{*}\right)\left[\left(c_{t-1}\left(\alpha_{t}\right)\right)\left(\left[\left(\bigwedge^{n-s} X\right)\left(\beta_{s}\left[e_{n}\right]\right)\right]\left(e_{n}\right)\right)\right] \\
= & (-1)^{t+r}(-1)^{n-s}\left(\beta_{s}\left[e_{n}\right]\right)\left[\left(\bigwedge^{n-1} X^{*}\right)\left(\left[c_{t-1}\left(\alpha_{t}\right)\right]\left[e_{n}\right]\right)\right]=-\left(c_{t-1} \otimes 1\right) * A(4) .
\end{aligned}
$$

It follows that $A(4)+B(4)=0$ and the proof is complete.

Remark 2.5. Suppose that the data of 1.6 is graded. Let $X$ be a homogeneous homomorphism of degree 1 , and let $u$ and $v$ be homogeneous elements of $F$ of degree $d_{u}$ and $d_{v}$, respectively. If $d_{u}+d_{v}=n-1$, then it is easy to check that $\mathbb{F}$ is a graded complex with homogeneous maps of degree zero, provided the grading on $\mathbb{F}$ is given by:

$$
\begin{aligned}
& \mathbb{F}_{r}(1)^{(t)}=R^{\left(\begin{array}{c}
n \\
t
\end{array}\right)\left(\begin{array}{c}
n \\
r+1-t
\end{array}\right)}\left[-\left(t n-t+d_{v}(r+1-2 t)\right)\right] \\
& \mathbb{F}_{r}(2)^{(t)}=R^{\left(\begin{array}{c}
n \\
t
\end{array}\right)\left(\begin{array}{c}
n \\
r-t
\end{array}\right)}\left[-\left(t n+d_{v}(r-2 t)\right)\right] \\
& \mathbb{F}_{r}(3)^{(t)}=R^{\left(\begin{array}{c}
n \\
t
\end{array}\right)\left(\begin{array}{c}
n \\
r-t
\end{array}\right)}\left[-\left((r-t) n+\left(d_{v}+1\right)(2 t-r)\right)\right] \\
& \left.\left.\mathbb{F}_{(4)}(t)=R^{\left(\begin{array}{c}
n \\
t
\end{array}\right)\left(\begin{array}{c}
n \\
r-1-t
\end{array}\right)[-((t+1) n-t+d(r-1-2 t)}\right)\right]
\end{aligned}
$$




\section{The complex $\mathbb{M}$.}

Theorem 3.1 is the main result in this section. Its proof appears after the proof of Proposition 3.14. When we want to emphasize the data which was used to construct $(\mathbb{M}, m)$, we write $\mathbb{M}[u, X, v]$. The case $n=2$ is handled in Proposition 3.20 .

Theorem 3.1. Adopt Data 1.6 with $3 \leq n$. Let $(\mathbb{M}, m)$ be the maps and modules of Definitions 3.7 and 3.12(c). The following statements hold.

(a) The maps and modules of $(\mathbb{M}, m$ ) form a complex

$$
\mathbb{M}: \quad 0 \rightarrow \mathbb{M}_{2 n} \rightarrow \ldots \rightarrow \mathbb{M}_{r} \stackrel{m_{r}}{\rightarrow} \mathbb{M}_{r-1} \rightarrow \ldots \rightarrow \mathbb{M}_{0}
$$

(b) Let $\mathbf{u}, \mathbf{X}$ and $\mathbf{v}$ be matrices which satisfy Convention 1.8. If $H$ is the ideal $H\left(\mathbf{u}, \mathbf{X},(-1)^{\frac{n(n-1)}{2}} \mathbf{v}\right)$ of Definition 1.1, then the homology $H_{0}(\mathbb{M})$ is equal to $R / H$,

(c) If $\mathbb{F}$ is the complex of Definition 2.1, then $H_{r}(\mathbb{F})=H_{r}(\mathbb{M})$ for all $r$.

(d) Each map $m_{r}$ of $\mathbb{M}$ satisfies $I_{1}\left(m_{r}\right) \subseteq I_{1}(u)+I_{1}(v)+I_{1}(X)$.

In section 4 we prove that $\mathbb{M}$ is acyclic whenever Data 1.6 is sufficiently generic (in the sense of Corollary 5.7). If, in addition, the data is local or graded (in the sense of Remark 2.5), then assertion (d) of the above result ensures that $\mathbb{M}$ is a minimal resolution. Some notation must be fixed before we can describe the modules of $\mathbb{M}$.

Definition 3.2. Adopt Data 1.6. For each integer $s$, let

$$
\mu_{s}: F \otimes \bigwedge^{s} F^{*} \rightarrow \bigwedge^{s-1} F^{*} \quad \text { and } \quad \sigma_{s}: \bigwedge^{s} F^{*} \rightarrow \bigwedge^{n-1} F \otimes \bigwedge^{s-1} F^{*}
$$

be the homomorphisms which are given by

$$
\mu_{s}\left(a_{1} \otimes \alpha_{s}\right)=a_{1}\left(\alpha_{s}\right) \quad \text { and } \quad \sigma_{s}\left(\alpha_{s}\right)=\sum_{i} \alpha_{1}^{[i]}\left(e_{n}\right) \otimes \alpha_{s-1}^{[i]}
$$

where $\Delta\left(\alpha_{s}\right)=\sum_{i} \alpha_{1}^{[i]} \otimes \alpha_{s-1}^{[i]}$.

Observation. Notice that $\mu_{s+1}$ is a surjection for all $s$, except $s=n$; and $\sigma_{s+1}$ is a split injection for all $s$, except $s=-1$.

Definition 3.3. Retain the notation of Definition 3.2. For each integer $s$, define homomorphisms

$$
\ell_{s}: \bigwedge^{s} F^{*} \rightarrow F \otimes \bigwedge^{s+1} F^{*} \quad \text { and } \quad \lambda_{s}: \bigwedge^{n-1} F \otimes \bigwedge^{s} F^{*} \rightarrow \bigwedge^{s+1} F^{*}, \quad \text { by }
$$

(a) $\ell_{s}$ is a fixed splitting of $\mu_{s+1}$ for $s \neq n$;

(b) $\ell_{n}=0$;

(c) $\lambda_{s}$ is a fixed splitting of $\sigma_{s+1}$ for $s \neq-1$; and

(d) $\lambda_{-1}=0$.

Remark 3.4. The maps $\ell_{s}$ and $\lambda_{s}$ have been chosen so that

$$
\begin{aligned}
& \mu_{s+1} \circ \ell_{s}=\mathrm{id} \quad \text { for all integers } s, \text { except } s=n, \text { and } \\
& \lambda_{s} \circ \sigma_{s+1}=\mathrm{id} \quad \text { for all } s, \text { except } s=-1 .
\end{aligned}
$$


Definition 3.5. Retain the notation of Definition 3.3 with $3 \leq n$. For $i=2$ and 3 , define submodules $\left[\mathbb{F}_{r}(i)^{(n-1)}\right]^{\prime}$ and $\left[\mathbb{F}_{r}(i)^{(n-1)}\right]^{\prime \prime}$ of $\mathbb{F}_{r}(i)^{(n-1)}$, and submodules $\left[\mathbb{F}_{r}(i)^{(1)}\right]^{\prime}$ and $\left[\mathbb{F}_{r}(i)^{(1)}\right]^{\prime \prime}$ of $\mathbb{F}_{r}(i)^{(1)}$ by

$$
\begin{aligned}
{\left[\mathbb{F}_{r}(i)^{(n-1)}\right]^{\prime} } & =\operatorname{Ker}\left[\mathbb{F}_{r}(i)^{(n-1)}=\bigwedge^{n-1} F \otimes \bigwedge^{r+1-n} F^{*} \stackrel{\lambda_{r+1-n}}{\longrightarrow} \bigwedge^{r+2-n} F^{*}\right], \\
{\left[\mathbb{F}_{r}(i)^{(n-1)}\right]^{\prime \prime} } & =\operatorname{Im}\left[\bigwedge^{r+2-n} F^{*} \stackrel{\sigma_{r+2-n}}{\longrightarrow} \bigwedge^{n-1} F \otimes \bigwedge^{r+1-n} F^{*}=\mathbb{F}_{r}(i)^{(n-1)}\right], \\
{\left[\mathbb{F}_{r}(i)^{(1)}\right]^{\prime} } & =\operatorname{Ker}\left[\mathbb{F}_{r}(i)^{(1)}=F \otimes \bigwedge^{r-1} F^{*} \stackrel{\mu_{r-1}}{\longrightarrow} \bigwedge^{r-2} F^{*}\right], \text { and } \\
{\left[\mathbb{F}_{r}(i)^{(1)}\right]^{\prime \prime} } & =\operatorname{Im}\left[\bigwedge^{r-2} F^{*} \stackrel{\ell_{r-2}}{\longrightarrow} F \otimes \bigwedge^{r-1} F^{*}=\mathbb{F}_{r}(i)^{(1)}\right] .
\end{aligned}
$$

The following statements are immediate consequences of Definition 3.5.

Observation 3.6. If $i=2$ or 3 , then

(a) $\mathbb{F}_{r}(i)^{(1)}=\left[\mathbb{F}_{r}(i)^{(1)}\right]^{\prime} \oplus\left[\mathbb{F}_{r}(i)^{(1)}\right]^{\prime \prime}$ for all $r$,

(b) $\mathbb{F}_{r}(i)^{(n-1)}=\left[\mathbb{F}_{r}(i)^{(n-1)}\right]^{\prime} \oplus\left[\mathbb{F}_{r}(i)^{(n-1)}\right]^{\prime \prime}$ for all $r$,

(c) $\left[\mathbb{F}_{r}(i)^{(1)}\right]^{\prime \prime}=0$, for $r \leq 1$,

(d) $\left[\mathbb{F}_{r}(i)^{(n-1)}\right]^{\prime \prime}=0$, for $2 n-1 \leq r$.

(e) $\left[\mathbb{F}_{r}(i)^{(1)}\right]^{\prime}=0$, for $n+1 \leq r$, and

(f) $\left[\mathbb{F}_{r}(i)^{(n-1)}\right]^{\prime}=0$, for $r \leq n-1$.

Definition 3.7. Adopt Data 1.6 with $3 \leq n$. The module $\mathbb{M}_{r}$ of $\mathbb{M}=\mathbb{M}[u, X, v]$ is obtained as follows. Let $\widehat{\mathbb{M}}_{r}$ represent the following submodule of $\mathbb{F}_{r}$ :

$$
\begin{aligned}
\widehat{\mathbb{M}}_{r}= & \sum_{t \notin\{0, n, r+1, r+1-n\}} \mathbb{F}_{r}(1)^{(t)}+\left[\mathbb{F}_{r}(2)^{(n-1)}\right]^{\prime}+\sum_{2 \leq t \leq n-2} \mathbb{F}_{r}(2)^{(t)}+\left[\mathbb{F}_{r}(2)^{(1)}\right]^{\prime} \\
& +\left[\mathbb{F}_{r}(3)^{(n-1)}\right]^{\prime}+\sum_{2 \leq t \leq n-2} \mathbb{F}_{r}(3)^{(t)}+\left[\mathbb{F}_{r}(3)^{(1)}\right]^{\prime}+\sum_{t \notin\{0, n, r-1, r-1-n\}} \mathbb{F}_{r}(4)^{(t)} .
\end{aligned}
$$

The submodule $\mathbb{M}_{r}$ of $\mathbb{F}_{r}$ is defined by

$$
\mathbb{M}_{r}= \begin{cases}\widehat{\mathbb{M}}_{0}+\mathbb{F}_{0}(3)^{(0)}, & \text { if } r=0, \\ \widehat{\mathbb{M}}_{2}+\left[\mathbb{F}_{2}(3)^{(1)}\right]^{\prime \prime}, & \text { if } r=2, \\ \widehat{\mathbb{M}}_{2 n-2}+\left[\mathbb{F}_{2 n-2}(3)^{(n-1)}\right]^{\prime \prime}, & \text { if } r=2 n-2, \\ \widehat{\mathbb{M}}_{2 n}+\mathbb{F}_{2 n}(3)^{(n)}, & \text { if } r=2 n, \text { and } \\ \widehat{\mathbb{M}}_{r}, & \text { for all other } r .\end{cases}
$$

Remark 3.8. Adopt the grading hypotheses of Remark 2.5. If $n=3$ and $d_{u}=d_{v}=$ 1 , then $\mathbb{M}$ is

$$
0 \rightarrow R(-9) \rightarrow R(-7)^{15} \rightarrow R(-6)^{35} \rightarrow \stackrel{R(-4)^{21}}{\oplus} \rightarrow R(-3)^{35} \rightarrow R(-2)^{15} \rightarrow R .
$$


If $n=4, d_{u}=1$, and $d_{v}=2$, then $\mathbb{M}$ is

$$
\begin{aligned}
& R(-6)^{10}
\end{aligned}
$$

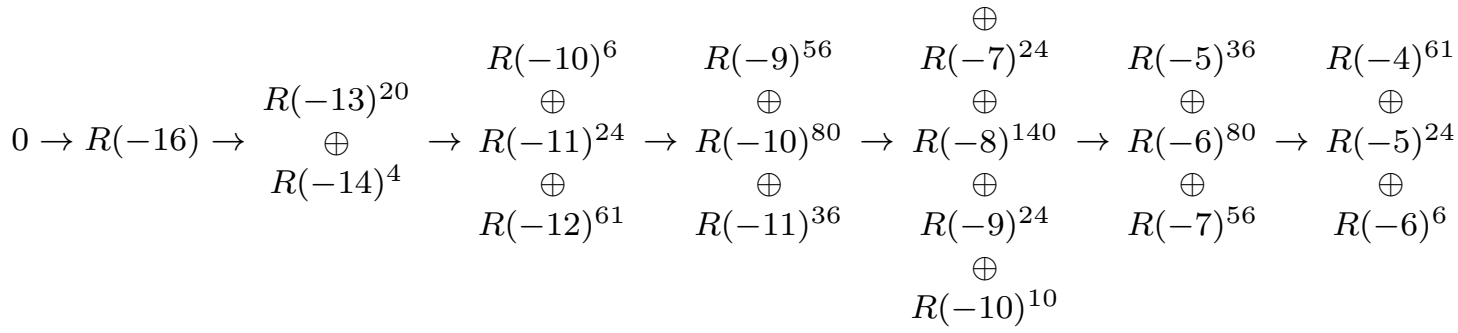

$$
\begin{aligned}
& \rightarrow \underset{R(-3)^{20}}{\oplus} \rightarrow R
\end{aligned}
$$

In general, if $4 \leq n$, then $\mathbb{M}_{0}=R$,

$$
\begin{aligned}
& \mathbb{M}_{1}=R^{n^{2}}[-(n-1)] \oplus R^{n}\left[-\left(1+d_{u}\right)\right] \oplus R^{n}\left[-\left(1+d_{v}\right)\right], \\
& \mathbb{M}_{2}=R\left(\begin{array}{c}
n \\
2
\end{array}\right) n\left[-\left(n-1+d_{u}\right)\right] \oplus R^{\left(\begin{array}{c}
n \\
2
\end{array}\right) n}\left[-\left(n-1+d_{v}\right)\right] \oplus R^{\left(\begin{array}{c}
n \\
2
\end{array}\right)}\left[-\left(2+2 d_{u}\right)\right] \oplus R^{2 n^{2}-1}[-n] \\
& \oplus R^{\left(\begin{array}{c}
n \\
2
\end{array}\right)}\left[-\left(2+2 d_{v}\right)\right] \\
& \mathbb{M}_{r}=\sum_{t=1}^{r} R^{\left(\begin{array}{c}
n \\
t
\end{array}\right)\left(\begin{array}{c}
n \\
r+1-t
\end{array}\right)}\left[-\left(t n-t+d_{v}(r+1-2 t)\right)\right] \oplus R^{n\left(\begin{array}{c}
n \\
r-1
\end{array}\right)-\left(\begin{array}{c}
n \\
r-2
\end{array}\right)}\left[-\left(n+d_{v}(r-2)\right)\right] \\
& \oplus \sum_{t=2}^{r} R^{\left(\begin{array}{c}
n \\
t
\end{array}\right)\left(\begin{array}{c}
n \\
r-t
\end{array}\right)}\left[-\left(t n+d_{v}(r-2 t)\right)\right] \oplus R^{n\left(\begin{array}{c}
n \\
r-1
\end{array}\right)-\left(\begin{array}{c}
n \\
r-2
\end{array}\right)}\left[-\left((r-1) n+\left(d_{v}+1\right)(2-r)\right)\right] \\
& \oplus \sum_{t=2}^{r} R^{\left(\begin{array}{c}
n \\
t
\end{array}\right)\left(\begin{array}{c}
n \\
r-t
\end{array}\right)}\left[-\left((r-t) n+\left(d_{v}+1\right)(2 t-r)\right)\right] \\
& \oplus \sum_{t=1}^{r-2} R^{\left(\begin{array}{c}
n \\
t
\end{array}\right)\left(\begin{array}{c}
n \\
r-1-t
\end{array}\right)}\left[-\left((t+1) n-t+d_{v}(r-1-2 t)\right)\right] \text { for } 3 \leq r \leq n-2, \\
& \mathbb{M}_{n-1}=\sum_{t=1}^{n-1} R^{\left(\begin{array}{c}
n \\
t
\end{array}\right)^{2}}\left[-\left(t n-t+d_{v}(n-2 t)\right)\right] \oplus R^{n\left(\begin{array}{c}
n \\
2
\end{array}\right)-\left(\begin{array}{c}
n \\
3
\end{array}\right)}\left[-\left(n+d_{v}(n-3)\right)\right] \\
& \oplus \sum_{t=2}^{n-2} R^{\left(\begin{array}{c}
n \\
t
\end{array}\right)\left(\begin{array}{c}
n \\
t+1
\end{array}\right)}\left[-\left(t n+d_{v}(n-1-2 t)\right)\right] \oplus R^{n\left(\begin{array}{c}
n \\
2
\end{array}\right)-\left(\begin{array}{c}
n \\
3
\end{array}\right)}\left[-\left(n+d_{u}(n-3)\right)\right] \\
& \oplus \sum_{t=2}^{n-2} R^{\left(\begin{array}{c}
n \\
t
\end{array}\right)\left(\begin{array}{c}
n \\
t+1
\end{array}\right)}\left[-\left((n-1-t) n+\left(d_{v}+1\right)(2 t-n+1)\right)\right] \\
& \oplus \sum_{t=1}^{n-3} R^{\left(\begin{array}{c}
n \\
t
\end{array}\right)\left(\begin{array}{c}
n \\
t+2
\end{array}\right)}\left[-\left((t+1) n-t+d_{v}(n-2-2 t)\right)\right], \text { and } \\
& \mathbb{M}_{n}=\sum_{t=2}^{n-1} R^{\left(\begin{array}{c}
n \\
t
\end{array}\right)\left(\begin{array}{c}
n \\
t-1
\end{array}\right)}\left[-\left(t n-t+d_{v}(n+1-2 t)\right)\right] \oplus R^{n^{2}-\left(\begin{array}{c}
n \\
2
\end{array}\right)}\left[-\left(n+d_{v}(n-2)\right)\right] \\
& \oplus \sum_{t=2}^{n-2} R^{\left(\begin{array}{c}
n \\
t
\end{array}\right)^{2}}\left[-\left(t n+d_{v}(n-2 t)\right)\right] \oplus R^{n^{2}-\left(\begin{array}{c}
n \\
2
\end{array}\right)}\left[-\left(2 n-2+d_{u}(n-2)\right)\right] \\
& \oplus R^{n^{2}-\left(\begin{array}{c}
n \\
2
\end{array}\right)}\left[-\left(n+d_{u}(n-2)\right)\right] \oplus \sum^{n-2} R^{\left(\begin{array}{l}
n \\
t
\end{array}\right)^{2}}\left[-\left((n-t) n+\left(d_{v}+1\right)(2 t-n)\right)\right]
\end{aligned}
$$




$$
\begin{aligned}
& \oplus R^{n^{2}-\left(\begin{array}{c}
n \\
2
\end{array}\right)}\left[-\left(2 n-2+d_{v}(n-2)\right)\right] \\
& \oplus \sum_{t=1}^{n-2} R^{\left(\begin{array}{c}
n \\
t
\end{array}\right)\left(\begin{array}{c}
n \\
t+1
\end{array}\right)}\left[-\left((t+1) n-t+d_{v}(n-1-2 t)\right)\right] .
\end{aligned}
$$

Furthermore, if $\mathbb{M}_{r}=\sum_{i} R^{b_{i}}\left[-m_{i}\right]$, then $\mathbb{M}_{2 n-r}=\sum_{i} R^{b_{i}}\left[-\left(n^{2}-m_{i}\right)\right]$.

Convention 3.9. For each statement "S", let

$$
\chi(\mathrm{S})= \begin{cases}1, & \text { if } \mathrm{S} \text { is true, and } \\ 0, & \text { if } \mathrm{S} \text { is false. }\end{cases}
$$

In particular, $\chi(i=j)$ has the same value as the Kronecker delta $\delta_{i j}$.

Definition 3.10. Adopt Data 1.6 with $3 \leq n$. For each integer $r$, let $\widehat{\mathbb{N}}_{r}$ be the following submodule of $\mathbb{F}_{r}$ :

$$
\begin{aligned}
\widehat{\mathbb{N}}_{r}= & \chi(n \leq r \leq 2 n-1) \cdot \mathbb{F}_{r}(1)^{(n)}+\chi(n \leq r \leq 2 n-2) \cdot \mathbb{F}_{r}(1)^{(r+1-n)} \\
& +\chi(0 \leq r \leq n) \cdot \mathbb{F}_{r}(2)^{(0)}+\chi(2 \leq r \leq n+1)\left[\mathbb{F}_{r}(2)^{(1)}\right]^{\prime \prime} \\
& +\chi(1 \leq r \leq n) \cdot \mathbb{F}_{r}(3)^{(0)}+\chi(3 \leq r \leq n+1) \cdot\left[\mathbb{F}_{r}(3)^{(1)}\right]^{\prime \prime} \\
& +\chi(n+1 \leq r \leq 2 n+1) \cdot \mathbb{F}_{r}(4)^{(n)}+\chi(n+1 \leq r \leq 2 n) \cdot \mathbb{F}_{r}(4)^{(r-1-n)} .
\end{aligned}
$$

Let $(\mathbb{N}, n)$ be the subcomplex of $(\mathbb{F}, f)$ which is given by

$$
\mathbb{N}_{r}=\widehat{\mathbb{N}}_{r}+f_{r+1}\left(\widehat{\mathbb{N}}_{r+1}\right) \text { and } n_{r}=\left.f_{r}\right|_{\mathbb{N}_{r}}
$$

For each integer $r$, let $\mathbb{L}_{r}$ be the following submodule of $\mathbb{F}_{r}$ :

$$
\begin{aligned}
\mathbb{L}_{r}= & \chi(-1 \leq r \leq n-1) \cdot \mathbb{F}_{r}(1)^{(0)}+\chi(0 \leq r \leq n-1) \cdot \mathbb{F}_{r}(1)^{(r+1)} \\
& +\chi(n-1 \leq r \leq 2 n-2) \cdot\left[\mathbb{F}_{r}(2)^{(n-1)}\right]^{\prime \prime}+\chi(n \leq r \leq 2 n) \cdot \mathbb{F}_{r}(2)^{(n)} \\
& +\chi(n-1 \leq r \leq 2 n-3) \cdot\left[\mathbb{F}_{r}(3)^{(n-1)}\right]^{\prime \prime}+\chi(n \leq r \leq 2 n-1) \cdot \mathbb{F}_{r}(3)^{(n)} \\
& +\chi(1 \leq r \leq n) \cdot \mathbb{F}_{r}(4)^{(0)}+\chi(2 \leq r \leq n) \cdot \mathbb{F}_{r}(4)^{(r-1)} .
\end{aligned}
$$

Remark 3.11. Use Observation 3.6 in order to see that

$$
\mathbb{F}_{r}=\mathbb{L}_{r} \oplus \mathbb{M}_{r} \oplus \widehat{\mathbb{N}}_{r} \quad \text { for all } r
$$

This decomposition gives rise to projection maps

$$
\pi_{r}^{\mathbb{L}}: \mathbb{F}_{r} \rightarrow \mathbb{L}_{r}, \quad \pi_{r}^{\mathbb{M}}: \mathbb{F}_{r} \rightarrow \mathbb{M}_{r}, \quad \text { and } \quad \pi_{r}^{\widehat{\mathbb{N}}}: \mathbb{F}_{r} \rightarrow \widehat{\mathbb{N}}_{r}
$$

For example, $\pi_{r}^{\mathbb{L}}$ is the map which annihilates $\mathbb{M}_{r} \oplus \widehat{\mathbb{N}}_{r}$, but restricts to give the 
Definition 3.12. Retain the notation of Definition 3.10.

(a) For each integer $r$, define $\tau_{r}: \mathbb{L}_{r} \rightarrow \widehat{\mathbb{N}}_{r+1}$ by

$$
\begin{aligned}
& \tau_{r}(1)^{(0)}\left(1 \otimes \beta_{r+1}\right)=(-1)^{r+1} 1 \otimes \beta_{r+1} \in \mathbb{F}_{r+1}(2)^{(0)}, \\
& \tau_{r}(1)^{(r+1)}\left(\alpha_{r+1} \otimes 1\right)=(-1)^{r} 1 \otimes \alpha_{r+1} \in \mathbb{F}_{r+1}(3)^{(0)}, \\
& \tau_{r}(2)^{(n-1)}\left(\sigma_{r+2-n}\left(\beta_{r+2-n}\right)\right)=\left\{\begin{array}{c}
\varepsilon_{n} \otimes \beta_{r+2-n} \in \mathbb{F}_{r+1}(1)^{(n)} \\
+ \\
\delta_{r n-1}(-1)^{n} v\left(\beta_{1}\right) \otimes \varepsilon_{n} \in \mathbb{F}_{n}(3)^{(0)},
\end{array}\right. \\
& \tau_{r}(2)^{(n)}\left(e_{n} \otimes \beta_{r-n}\right)=\left\{\begin{array}{c}
(-1)^{r+1} \varepsilon_{n} \otimes \beta_{r-n} \in \mathbb{F}_{r+1}(4)^{(n)} \\
+\quad \text { for } n-1 \leq r \leq 2 n-2, \\
\delta_{r n} \beta_{0} \cdot \ell_{n-1}\left[u\left(\varepsilon_{n}\right)\right] \in\left[\mathbb{F}_{n+1}(3)^{(1)}\right]^{\prime \prime},
\end{array}\right. \\
& \tau_{r}(3)^{(n-1)}\left(\sigma_{r+2-n}\left(\beta_{r+2-n}\right)\right)=\left\{\begin{array}{c}
\beta_{r+2-n} \otimes \varepsilon_{n} \in \mathbb{F}_{r+1}(1)^{(r+2-n)} \\
-\delta_{r n-1} u\left(\beta_{1}\right) \otimes \varepsilon_{n} \in \mathbb{F}_{n}(2)^{(0)},
\end{array}\right. \\
& \tau_{r}(3)^{(n)}\left(e_{n} \otimes \beta_{r-n}\right)=\left\{\begin{array}{c}
(-1)^{r+1} \beta_{r-n} \otimes \varepsilon_{n} \in \mathbb{F}_{r+1}(4)^{(r-n)} \\
+ \\
\delta_{r n}(-1)^{n} \beta_{0} \cdot \ell_{n-1}\left[v\left(\varepsilon_{n}\right)\right] \in\left[\mathbb{F}_{n+1}(2)^{(1)}\right]
\end{array}\right.
\end{aligned}
$$

(b) For each integer $r$, define $\psi_{r}: \mathbb{F}_{r} \rightarrow \mathbb{M}_{r}$ by

$$
\left.\psi_{r}\right|_{\mathbb{M}_{r}}=\mathrm{id},\left.\quad \psi_{r}\right|_{\widehat{\mathbb{N}}_{r}}=0 \quad \text { and }\left.\quad \psi_{r}\right|_{\mathbb{L}_{r}}=-\pi_{r}^{\mathbb{M}} \circ f_{r+1} \circ \tau_{r} .
$$

(c) For each integer $r$, define $m_{r}: \mathbb{M}_{r} \rightarrow \mathbb{M}_{r-1}$ to be the composition

$$
\mathbb{M}_{r} \stackrel{\text { incl }}{\longrightarrow} \mathbb{F}_{r} \stackrel{f_{r}}{\longrightarrow} \mathbb{F}_{r-1} \stackrel{\psi_{r-1}}{\longrightarrow} \mathbb{M}_{r-1}
$$

(d) For each integer $r$, define $\rho_{r}: \mathbb{M}_{r} \rightarrow \mathbb{F}_{r}$ by

$$
\rho_{r}=\operatorname{incl}_{r}-\tau_{r-1} \circ \pi_{r-1}^{\mathbb{L}} \circ f_{r} .
$$

Note. The definition of $\tau_{r}(i)^{(n-1)}$, for $i=2$ or 3 , is legitimate because Remark 3.4 guarantees that $\lambda_{r+1-n} \circ \sigma_{r+2-n}=\mathrm{id}$, provided $n-1 \leq r$.

The technical part of the proof of Theorem 3.1 is contained in the proof of the next result.

Lemma 3.13. In the notation of Definition 3.12, the maps

$$
\mathbb{L}_{r} \stackrel{\tau_{r}}{\longrightarrow} \widehat{\mathbb{N}}_{r+1} \quad \text { and } \quad \widehat{\mathbb{N}}_{r+1} \stackrel{\text { incl }}{\longrightarrow} \mathbb{F}_{r+1} \stackrel{f_{r+1}}{\longrightarrow} \mathbb{F}_{r} \stackrel{\pi_{r}^{\mathbb{L}}}{\longrightarrow} \mathbb{L}_{r}
$$

are inverses of one another.

Proof. We show that $\pi_{r}^{\mathbb{L}} \circ f_{r+1} \circ \tau_{r}(x)=x$ for all $x \in \mathbb{L}_{r}$ and $\tau_{r} \circ \pi_{r}^{\mathbb{L}} \circ f_{r+1}(y)=y$ for all $y \in \widehat{\mathbb{N}}_{r+1}$. There are eight cases. We first fix an integer $r$, with $-1 \leq r \leq n-1$. Let $x=1 \otimes \beta_{r+1} \in \mathbb{F}_{r}(1)^{(0)}$ and $y=1 \otimes \beta_{r+1} \in \mathbb{F}_{r+1}(2)^{(0)}$. Observe that 
In the second case, we take $0 \leq r \leq n-1$. Let $x=\alpha_{r+1} \otimes 1 \in \mathbb{F}_{r}(1)^{(r+1)}$ and $y$ equal $1 \otimes \alpha_{r+1} \in \mathbb{F}_{r+1}(3)^{(0)}$. Observe that

$$
\tau_{r}(x)=(-1)^{r} y \quad \text { and } \quad \pi_{r}^{\mathbb{L}} \circ f_{r+1}(y)=(-1)^{r} x .
$$

In the third case, we have $n-1 \leq r \leq 2 n-2$. Let $x=\sigma_{r+2-n}\left(\beta_{r+2-n}\right) \in$ $\left[\mathbb{F}_{r}(2)^{(n-1)}\right]^{\prime \prime}$ and $y=\varepsilon_{n} \otimes \beta_{r+2-n} \in \mathbb{F}_{r+1}(1)^{(n)}$. If $x^{\prime}=\delta_{r n-1} \cdot \varepsilon_{n} \otimes v\left(\beta_{1}\right) \in$ $\mathbb{F}_{n-1}(1)^{(n)}$ and $y^{\prime}$ is equal to $\delta_{r n-1} \cdot v\left(\beta_{1}\right) \otimes \varepsilon_{n} \in \mathbb{F}_{n}(3)^{(0)}$, then

$\tau_{r}(x)=y+(-1)^{n} y^{\prime}, \quad \tau_{r}\left(x^{\prime}\right)=(-1)^{n-1} y^{\prime}, \quad \pi_{r}^{\mathbb{L}} \circ f_{r+1}(y)=x+x^{\prime}, \quad$ and $\quad \pi_{r}^{\mathbb{L}} \circ f_{r+1}\left(y^{\prime}\right)=(-1)^{n-1} x^{\prime}$.

In the fourth case, we consider $n \leq r \leq 2 n$. Let $x=e_{n} \otimes \beta_{r-n} \in \mathbb{F}_{r}(2)^{(n)}$ and $y$ equal $\varepsilon_{n} \otimes \beta_{r-n} \in \mathbb{F}_{r+1}(4)^{(n)}$. If $\bar{x}^{\prime}=\delta_{r n} \cdot u\left(\varepsilon_{n}\right) \otimes \beta_{0} \in \mathbb{F}_{n}(4)^{(n-1)}$ and $y^{\prime}=\delta_{r n} \cdot \beta_{0} \cdot \ell_{n-1}\left[u\left(\varepsilon_{n}\right)\right]$ in $\left[\mathbb{F}_{n+1}(3)^{(1)}\right]^{\prime \prime}$, then

$\tau_{r}(x)=(-1)^{r+1} y+y^{\prime}, \quad \tau_{r}\left(x^{\prime}\right)=-y^{\prime}, \quad \pi_{r}^{\mathbb{L}} \circ f_{r+1}(y)=(-1)^{r+1} x+(-1)^{r+1} x^{\prime}, \quad$ and $\pi_{r}^{\mathbb{L}} \circ f_{r+1}\left(y^{\prime}\right)=-x^{\prime}$.

In case five, we have $n-1 \leq r \leq 2 n-3$. Let $x=\sigma_{r+2-n}\left(\beta_{r+2-n}\right) \in\left[\mathbb{F}_{r}(3)^{(n-1)}\right]^{\prime \prime}$ and $y=\beta_{r+2-n} \otimes \varepsilon_{n} \in \mathbb{F}_{r+1}(1)^{(r+2-n)}$. If $x^{\prime}=\delta_{r n-1} \cdot u\left(\beta_{1}\right) \otimes \varepsilon_{n} \in \mathbb{F}_{n-1}(1)^{(0)}$ and $y^{\prime}=\delta_{r n-1} \cdot u\left(\beta_{1}\right) \otimes \varepsilon_{n} \in \mathbb{F}_{n}(2)^{(0)}$, then

$\tau_{r}(x)=y-y^{\prime}, \quad \tau_{r}\left(x^{\prime}\right)=(-1)^{n} y^{\prime}, \quad \pi_{r}^{\mathbb{L}} \circ f_{r+1}(y)=x+(-1)^{n} x^{\prime}, \quad$ and $\quad \pi_{r}^{\mathbb{L}} \circ f_{r+1}\left(y^{\prime}\right)=(-1)^{n} x^{\prime}$.

In the sixth case, we consider $n \leq r \leq 2 n-1$. Let $x=e_{n} \otimes \beta_{r-n} \in \mathbb{F}_{r}(3)^{(n)}$ and $y=\beta_{r-n} \otimes \varepsilon_{n} \in \mathbb{F}_{r+1}(4)^{(r-n)}$. If $x^{\prime}=\delta_{r n} \cdot \beta_{0} \otimes v\left(\varepsilon_{n}\right) \in \mathbb{F}_{n}(4)^{(0)}$ and $y^{\prime}$ is equal to $\delta_{r n} \cdot \beta_{0} \cdot \ell_{n-1}\left(v\left[\varepsilon_{n}\right]\right) \in\left[\mathbb{F}_{n+1}(2)^{(1)}\right]^{\prime \prime}$, then

$\tau_{r}(x)=(-1)^{r+1} y+(-1)^{n} y^{\prime}, \quad \tau_{r}\left(x^{\prime}\right)=y^{\prime}, \quad \pi_{r}^{\mathbb{L}} \circ f_{r+1}(y)=(-1)^{r+1} x+x^{\prime}, \quad$ and $\quad \pi_{r}^{\mathbb{L}} \circ f_{r+1}\left(y^{\prime}\right)=x^{\prime}$.

In case seven, we have $1 \leq r \leq n$. Let $x=1 \otimes \beta_{r-1} \in \mathbb{F}_{r}(4)^{(0)}$ and $y=\ell_{r-1}\left(\beta_{r-1}\right)$ in $\left[\mathbb{F}_{r+1}(2)^{(1)}\right]^{\prime \prime}$. Observe that $\tau_{r}(x)=y$ and $\pi_{r}^{\mathbb{L}} \circ f_{r+1}(y)=x$. Finally, we take $2 \leq r \leq n$. Let $x=\alpha_{r-1} \otimes 1 \in \mathbb{F}_{r}(4)^{(r-1)}$ and $y=\ell_{r-1}\left(\alpha_{r-1}\right) \in\left[\mathbb{F}_{r+1}(3)^{(1)}\right]^{\prime \prime}$. The proof is complete because $\tau_{r}(x)=-y$ and $\pi_{r}^{\mathbb{L}} \circ f_{r+1}(y)=-x$.

Proposition 3.14. Adopt Data 1.6 with $3 \leq n$. Let $(\mathbb{F}, f)$ be the complex of Definition 2.1 and $(\mathbb{N}, n)$ be the subcomplex of $\mathbb{F}$ of Definition 3.10.

(a) The complex $(\mathbb{N}, n)$ is split exact.

(b) The modules and maps $\left\{m_{r}: \mathbb{M}_{r} \rightarrow \mathbb{M}_{r-1}\right\}$ of Definitions 3.7 and 3.12(c) form a complex, which we denote (M, $m$ ).

(c) The maps $\left\{\psi_{r}: \mathbb{F}_{r} \rightarrow \mathbb{M}_{r}\right\}$ of Definition 3.12(b) form a map of complexes; furthermore,

$$
0 \rightarrow(\mathbb{N}, n) \stackrel{\text { incl }}{\longrightarrow}(\mathbb{F}, f) \stackrel{\psi}{\longrightarrow}(\mathbb{M}, m) \rightarrow 0
$$

is a short exact sequence of complexes.

(d) The maps $\left\{\rho_{r}: \mathbb{M}_{r} \rightarrow \mathbb{F}_{r}\right\}$ of Definition 3.12(d) form a map of complexes; furthermore, the composition

$$
\mathbb{M}_{r} \stackrel{\rho_{r}}{\longrightarrow} \mathbb{F}_{r} \stackrel{\psi_{r}}{\longrightarrow} \mathbb{M}_{r}
$$


Proof. Let $s_{r}: \mathbb{F}_{r} \rightarrow \mathbb{F}_{r+1}$ be the map which is given by

$$
\left.s_{r}\right|_{\mathbb{L}_{r}}=\tau_{r},\left.\quad s_{r}\right|_{\mathbb{M}_{r}}=0, \quad \text { and }\left.\quad s_{r}\right|_{\widehat{\mathbb{N}}_{r}}=0
$$

It follows, from Lemma 3.13, that

$$
\left.s_{r}\right|_{\widehat{\mathbb{N}}_{r}}=0 \quad \text { and }\left.\quad s_{r-1} \circ f_{r}\right|_{\widehat{\mathbb{N}}_{r}}=\left.\mathrm{id}\right|_{\widehat{\mathbb{N}}_{r}}
$$

Assertion (a) is established because the maps $\left.s_{r}\right|_{\mathbb{N}_{r}}$ form a homotopy on $\mathbb{N}$ in the sense that

$$
\left.s_{r-1}\right|_{\mathbb{N}_{r-1}} \circ n_{r}+\left.n_{r+1} \circ s_{r}\right|_{\mathbb{N}_{r}}=\left.\mathrm{id}\right|_{\mathbb{N}_{r}} \text { for all } r .
$$

We next show that

$$
\mathbb{M}_{r}+\mathbb{N}_{r}=\mathbb{F}_{r} \quad \text { for all } r
$$

If $x_{r} \in \mathbb{L}_{r}$, then Lemma 3.13 gives $x_{r}=\pi_{r}^{\mathbb{L}} \circ f_{r+1} \circ \tau_{r}\left(x_{r}\right)$; and therefore, $x_{r}-$ $f_{r+1}\left(\tau_{r}\left(x_{r}\right)\right)$ is in $\mathbb{M}_{r}+\widehat{\mathbb{N}}_{r}$. It follows that

$$
\mathbb{L}_{r} \subseteq \mathbb{M}_{r}+\widehat{\mathbb{N}}_{r}+f_{r+1}\left(\widehat{\mathbb{N}}_{r+1}\right)=\mathbb{M}_{r}+\mathbb{N}_{r}
$$

and (3.15) is established by Remark 3.11. Now we prove (b). Observe that $m_{r}=\pi_{r-1}^{\mathbb{M}}\left(1-f_{r} \circ \tau_{r-1} \circ \pi_{r-1}^{\mathbb{L}}\right) \circ f_{r} \quad$ and $\quad m_{r+1}=\pi_{r}^{\mathbb{M}} \circ f_{r+1}\left(1-\tau_{r} \circ \pi_{r}^{\mathbb{L}} \circ f_{r+1}\right)$

We know, from Remark 3.11, that

$$
\left.\mathrm{id}\right|_{\mathbb{F}_{r}}=\pi_{r}^{\mathbb{L}}+\pi_{r}^{\mathbb{M}}+\pi_{r}^{\widehat{\mathbb{N}}} \quad \text { and } \quad f_{r} \circ f_{r+1}=0
$$

therefore, we see that

$f_{r} \circ m_{r+1}=f_{r} \circ \pi_{r}^{\mathbb{M}} \circ f_{r+1}+f_{r} \circ\left(\pi_{r}^{\mathbb{L}} \circ f_{r+1} \circ \tau_{r}\right) \circ \pi_{r}^{\mathbb{L}} \circ f_{r+1}+f_{r} \circ \pi_{r}^{\widehat{\mathbb{N}}} \circ f_{r+1} \circ \tau_{r} \circ \pi_{r}^{\mathbb{L}} \circ f_{r+1}$.

Apply Lemma 3.13 to see that the expression inside the parentheses is the identity map, and apply (3.16) to see that

$$
f_{r} \circ m_{r+1}=f_{r} \circ \pi_{r}^{\widehat{\mathbb{N}}} \circ f_{r+1} \circ\left(-1+\tau_{r} \circ \pi_{r}^{\mathbb{L}} \circ f_{r+1}\right) .
$$

Thus, we have

$$
\begin{aligned}
m_{r} \circ m_{r+1} & =\pi_{r-1}^{\mathbb{M}}\left(1-f_{r} \circ \tau_{r-1} \circ \pi_{r-1}^{\mathbb{L}}\right) \circ f_{r} \circ m_{r+1} \\
& =\pi_{r-1}^{\mathbb{M}}\left(1-f_{r} \circ \tau_{r-1} \circ \pi_{r-1}^{\mathbb{L}}\right) \circ f_{r} \circ \pi_{r}^{\widehat{\mathbb{N}}} \circ f_{r+1} \circ\left(-1+\tau_{r} \circ \pi_{r}^{\mathbb{L}} \circ f_{r+1}\right) \\
& =\pi_{r-1}^{\mathbb{M}} \circ f_{r} \circ\left[\pi_{r}^{\widehat{\mathbb{N}}}-\tau_{r-1} \circ \pi_{r-1}^{\mathbb{L}} \circ f_{r} \circ \pi_{r}^{\widehat{\mathbb{N}}}\right] \circ f_{r+1} \circ\left(-1+\tau_{r} \circ \pi_{r}^{\mathbb{L}} \circ f_{r+1}\right)
\end{aligned}
$$

Apply Lemma 3.13, once again, to see that the expression inside the brackets is 
The interesting part of the proof of $\mathbb{N}_{r} \subseteq \operatorname{ker} \psi_{r}$ is

$$
\begin{aligned}
\psi_{r} \circ f_{r+1} \circ \pi_{r+1}^{\widehat{\mathbb{N}}} & =\left(\pi_{r}^{\mathbb{M}}-\pi_{r}^{\mathbb{M}} \circ f_{r+1} \circ \tau_{r} \circ \pi_{r}^{\mathbb{L}}\right) \circ f_{r+1} \circ \pi_{r+1}^{\widehat{\mathbb{N}}} \\
& =\pi_{r}^{\mathbb{M}} \circ f_{r+1} \circ\left(1-\tau_{r} \circ \pi_{r}^{\mathbb{L}} \circ f_{r+1}\right) \circ \pi_{r+1}^{\widehat{\mathbb{N}}} .
\end{aligned}
$$

Lemma 3.13 ensures that the expression inside the parentheses is zero. To prove that $\psi: \mathbb{F} \rightarrow \mathbb{M}$ is a map of complexes, we must show that

$$
m_{r} \circ \psi_{r}\left(x_{r}\right)=\psi_{r-1} \circ f_{r}\left(x_{r}\right)
$$

for all $x_{r} \in \mathbb{F}_{r}$. If $x_{r} \in \mathbb{M}_{r}$, then the left side of (3.17) is

$$
m_{r}\left(x_{r}\right)=\psi_{r-1} \circ f_{r}\left(x_{r}\right) .
$$

If $x_{r} \in \mathbb{N}_{r}$, then both sides of (3.17) are zero; and therefore, (3.17) is established by (3.15). We complete the proof of (c) by identifying the kernel of $\psi$. Let $x_{r} \in \operatorname{Ker} \psi_{r}$. Use (3.15) to write $x_{r}=y_{r}+z_{r}$ for some $y_{r} \in \mathbb{M}_{r}$ and some $z_{r} \in \mathbb{N}_{r}$. Observe that

$$
0=\psi_{r}\left(x_{r}\right)=\psi_{r}\left(y_{r}\right)+\psi_{r}\left(z_{r}\right)=y_{r} .
$$

We conclude that ker $\psi_{r}=\mathbb{N}_{r}$ and the proof of (c) is complete.

We conclude by proving $(\mathrm{d})$. It is clear that $\psi_{r} \circ \rho_{r}=\mathrm{id}$. The proof that $\rho$ is a map of complexes is much like the proof of (b). We see that $\rho_{r-1} \circ m_{r}$ is equal to

$$
\rho_{r-1} \circ \pi_{r-1}^{\mathbb{M}} \circ f_{r}-\rho_{r-1} \circ \pi_{r-1}^{\mathbb{M}} \circ f_{r} \circ \tau_{r-1} \circ \pi_{r-1}^{\mathbb{L}} \circ f_{r} .
$$

The second term of (3.18) is equal to

$$
-\pi_{r-1}^{\mathbb{M}} \circ f_{r} \circ \tau_{r-1} \circ \pi_{r-1}^{\mathbb{L}} \circ f_{r}+\tau_{r-2} \circ \pi_{r-2}^{\mathbb{L}} \circ f_{r-1} \circ \pi_{r-1}^{\mathbb{M}} \circ f_{r} \circ \tau_{r-1} \circ \pi_{r-1}^{\mathbb{L}} \circ f_{r} .
$$

Use (3.16) to see that the second term of (3.18) is

$$
\begin{aligned}
& -\pi_{r-1}^{\mathbb{M}} \circ f_{r} \circ \tau_{r-1} \circ \pi_{r-1}^{\mathbb{L}} \circ f_{r}-\left[\tau_{r-2} \circ \pi_{r-2}^{\mathbb{L}} \circ f_{r-1}\right] \circ \pi_{r-1}^{\widehat{\mathbb{N}}} \circ f_{r} \circ \tau_{r-1} \circ \pi_{r-1}^{\mathbb{L}} \circ f_{r} \\
& -\tau_{r-2} \circ \pi_{r-2}^{\mathbb{L}} \circ f_{r-1} \circ\left[\pi_{r-1}^{\mathbb{L}} \circ f_{r} \circ \tau_{r-1}\right] \circ \pi_{r-1}^{\mathbb{L}} \circ f_{r} .
\end{aligned}
$$

Lemma 3.13 guarantees that each bracketed expression is equal to the identity map. Apply (3.16) two times to see that the second term of (3.18) is equal to

$$
\begin{aligned}
& {\left[\pi_{r-1}^{\mathbb{L}} \circ f_{r} \circ \tau_{r-1}\right] \circ \pi_{r-1}^{\mathbb{L}} \circ f_{r}-f_{r} \circ \tau_{r-1} \circ \pi_{r-1}^{\mathbb{L}} \circ f_{r}+\left[\tau_{r-2} \circ \pi_{r-2}^{\mathbb{L}} \circ f_{r-1}\right] \circ \pi_{r-1}^{\widehat{\mathbb{N}}} \circ f_{r}} \\
& +\tau_{r-2} \circ \pi_{r-2}^{\mathbb{L}} \circ f_{r-1} \circ \pi_{r-1}^{\mathbb{M}} \circ f_{r} .
\end{aligned}
$$

Once more, Lemma 3.13 ensures that each bracketed expression is the identity map. Thus, the second term of (3.18) is 
The definition of $\rho$ yields that the first term of (3.18) is

$$
\pi_{r-1}^{\mathbb{M}} \circ f_{r}-\tau_{r-2} \circ \pi_{r-2}^{\mathbb{L}} \circ f_{r-1} \circ \pi_{r-1}^{\mathbb{M}} \circ f_{r} .
$$

Combine the two most recent expressions to see that

$$
\rho_{r-1} \circ m_{r}=f_{r}-f_{r} \circ \tau_{r-1} \circ \pi_{r-1}^{\mathbb{L}} \circ f_{r}=f_{r} \circ\left(\text { incl }-\tau_{r-1} \circ \pi_{r-1}^{\mathbb{L}} \circ f_{r}\right)=f_{r} \circ \rho_{r},
$$

and the proof is complete.

Proof of Theorem 3.1. Assertions (a) and (c) are contained in Proposition 3.14. To prove (b), we use Lemma 1.9 and the notation of Convention 1.8 to see that

$$
\begin{aligned}
m_{1}(1)^{(1)}\left(\varepsilon_{1}^{[j]} \otimes \varepsilon_{1}^{[i]}\right) & =-u\left(\varepsilon_{1}^{[j]}\right) \cdot v\left(\varepsilon_{1}^{[i]}\right)+\left[\varepsilon_{1}^{[j]} \wedge\left(\bigwedge^{n-1} X\right)\left(\varepsilon_{1}^{[i]}\left[e_{n}\right]\right)\right]\left(e_{n}\right) \\
& =-u_{j} v_{i}+(-1)^{\frac{n(n-1)}{2}}(\operatorname{Adj} \mathbf{X})_{i j} \\
& =-(-1)^{\frac{n(n-1)}{2}}\left[(-1)^{\frac{n(n-1)}{2}} \mathbf{v u}-\operatorname{Adj} \mathbf{X}\right]_{i j} \\
m_{1}(2)^{(1)}\left(e_{1}^{[j]} \otimes 1\right) & =u\left[X\left(e_{1}^{[j]}\right)\right]=\sum_{i} u_{i} x_{i j} \\
m_{1}(3)^{(1)}\left(e_{1}^{[i]} \otimes 1\right) & =[X(v)]\left(e_{1}^{[i]}\right)=\sum_{j} x_{i j} v_{j} .
\end{aligned}
$$

A straightforward calculation using Definitions 2.1 and 3.7 shows that $m_{0}\left(\mathbb{M}_{0}\right)=0$,

$$
f_{r}\left(\mathbb{M}_{r}\right) \subseteq\left(I_{1}(\mathbf{X})+I_{1}(\mathbf{u})+I_{1}(\mathbf{v})\right) \cdot \mathbb{F}_{r-1}, \quad \text { provided } r \neq 0 \text { and } r \neq 2, \text { and }
$$

$f_{2}\left(\widehat{\mathbb{M}}_{2}\right)+m_{2}\left(\left[\mathbb{F}_{2}(3)^{(1)}\right]^{\prime \prime}\right) \subseteq\left(I_{1}(\mathbf{X})+I_{1}(\mathbf{u})+I_{1}(\mathbf{v})\right) \cdot \mathbb{F}_{1}$. Assertion $(\mathrm{d})$ is established and the proof is complete.

The next result, which used in the proof of Theorem 5.2, is a small piece of the fact that the complex $\mathbb{M}$ is self dual.

Observation 3.19. If $(\mathbb{M}, m)$ is the complex of Theorem 3.1, then $I_{1}\left(m_{1}\right)=$ $I_{1}\left(m_{2 n}\right)$.

Proof. The back of $\mathbb{M}$ looks like

$$
0 \rightarrow \mathbb{M}_{2 n}=\mathbb{F}_{2 n}(3)^{(n)}=\bigwedge^{n} F \otimes \bigwedge^{n} F^{*} \stackrel{m_{2 n}}{\longrightarrow} \mathbb{M}_{2 n-1}=\left\{\begin{array}{c}
{\left[\mathbb{F}_{2 n-1}(2)^{(n-1)}\right]^{\prime}=\bigwedge^{n-1} F \otimes \bigwedge^{n} F^{*}} \\
{\left[\mathbb{F}_{2 n-1}(3)^{(n-1)}\right]^{\prime}=\bigwedge^{n-1} F \otimes \bigwedge^{n} F^{*}} \\
\mathbb{F}_{2 n-1}(4)^{(n-1)}=\bigwedge^{n-1} F^{*} \otimes \Lambda^{n-1} F^{*} .
\end{array}\right.
$$

Adopt the notation of Convention 1.8. One can readily check that

$$
m_{2 n}\left(e_{n} \otimes \varepsilon_{n}\right)=\left\{\begin{aligned}
{\left[X^{*}(u)\right]\left(e_{n}\right) \otimes \varepsilon_{n} } & \in\left[\mathbb{F}_{2 n-1}(2)^{(n-1)}\right]^{\prime} \\
& + \\
{[X(v)]\left(e_{n}\right) \otimes \varepsilon_{n} } & \in\left[\mathbb{F}_{2 n-1}(3)^{(n-1)}\right]^{\prime} \\
& +
\end{aligned}\right.
$$


where

$y=u\left(\varepsilon_{n}\right) \otimes v\left(\varepsilon_{n}\right)-\sum_{k}(-1)^{k+1} e_{1}^{[k]}\left(\varepsilon_{n}\right) \otimes\left(\bigwedge^{n-1} X^{*}\right)\left(e_{1}^{[1]} \wedge \ldots \wedge e_{1}^{\widehat{[k]}} \wedge \ldots \wedge e_{1}^{[n]}\right)$.

For fixed integers $i$ and $j$, consider the homomorphism

$$
\varphi_{i j}: \bigwedge^{n-1} F^{*} \otimes \bigwedge^{n-1} F^{*} \rightarrow \bigwedge^{n} F^{*} \otimes \bigwedge^{n} F^{*}
$$

which is given by

$$
\varphi_{i j}\left(\alpha_{n-1} \otimes \beta_{n-1}\right)=\varepsilon_{1}^{[i]} \wedge \alpha_{n-1} \otimes \varepsilon_{1}^{[j]} \wedge \beta_{n-1}
$$

A short calculation, using Proposition 1.2, yields

$$
\varphi_{i j}(y)=(-1)^{\frac{n(n-1)}{2}}\left[(-1)^{\frac{n(n-1)}{2}} \mathbf{v u}-\operatorname{Adj} \mathbf{X}\right]_{j i} \cdot \varepsilon_{n} \otimes \varepsilon_{n} \in \bigwedge^{n} F^{*} \otimes \bigwedge^{n} F^{*} .
$$

Proposition 3.20. Adopt Data 1.6 with $n=2$, and let $\mathbf{u}, \mathbf{X}, \mathbf{v}$ be the matrices of Convention 1.8. If $\mathbb{F}$ is the complex of Definition 2.1, then there is a split exact subcomplex $\mathbb{N}$ of $\mathbb{F}$ such that $\mathbb{F} / \mathbb{N}$ is the Koszul complex on the entries of Adj $\mathbf{X}+\mathbf{v u . ~}$

Proof. The proof is very similar to the proof of Theorem 3.1. The main difference is due to the fact that $1=n-1$; and therefore, we must replace the modules of Definition 3.5 with

$$
\left[\mathbb{F}_{2}(2)^{(1)}\right]^{\prime}=\operatorname{ker} \mu_{1}, \quad\left[\mathbb{F}_{2}(2)^{(1)}\right]^{\prime \prime}=\operatorname{im} \ell_{0}, \quad\left[\mathbb{F}_{2}(3)^{(1)}\right]^{\prime}=\operatorname{ker} \lambda_{1}, \quad \text { and } \quad\left[\mathbb{F}_{2}(3)^{(1)}\right]^{\prime \prime}=\operatorname{im} \sigma_{2} \text {. }
$$

Decompose $\mathbb{F}$ as $\mathbb{L} \oplus \mathbb{M} \oplus \widehat{\mathbb{N}}$, where

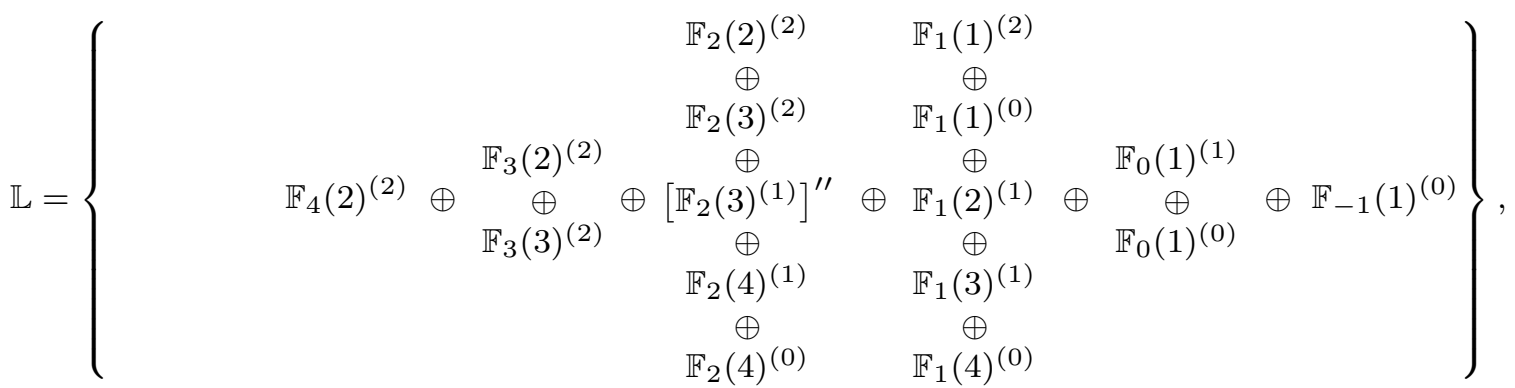

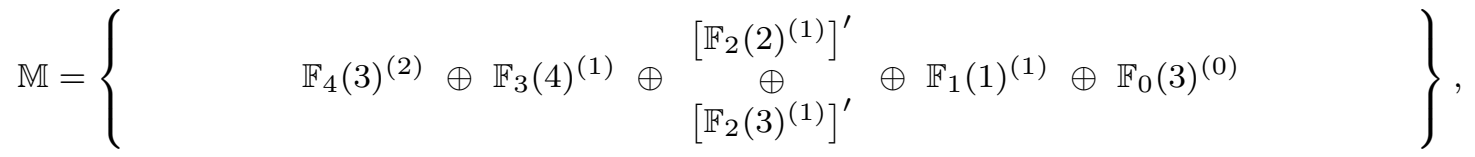

and

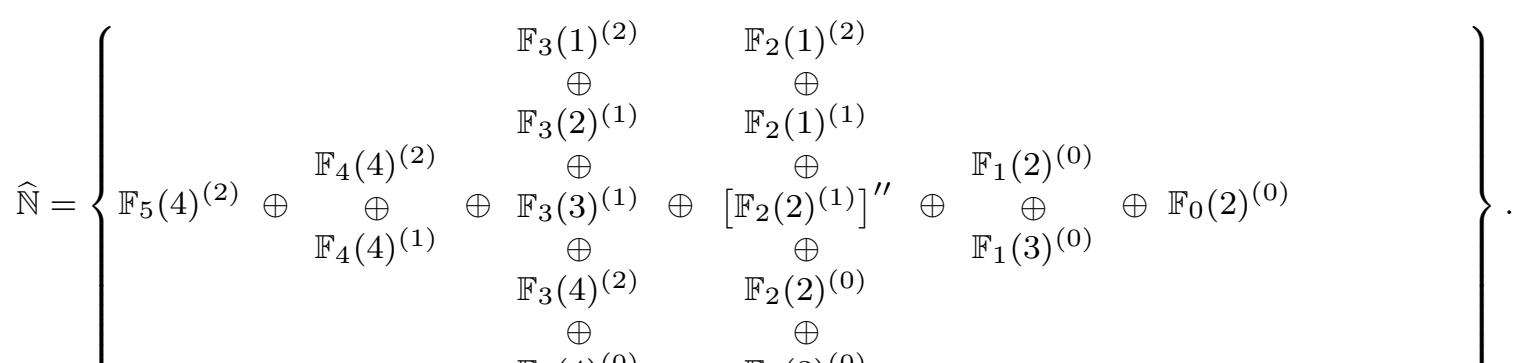


Let $(\mathbb{N}, n)$ be the subcomplex of $\mathbb{F}$ which is given by

$$
\mathbb{N}_{r}=\widehat{\mathbb{N}}_{r}+f_{r+1}\left(\widehat{\mathbb{N}}_{r+1}\right) \text { and } n_{r}=\left.f_{r}\right|_{\mathbb{N}_{r}}
$$

In order to simplify the rest of the argument, we take $\ell_{0}(1)$ to be the element $e_{1}^{[1]} \otimes \varepsilon_{1}^{[1]}$ of $\mathbb{F}_{2}(2)^{(1)}$, and $\lambda_{1}: \bigwedge^{1} F \otimes \bigwedge^{1} F^{*} \rightarrow \bigwedge^{2} F^{*}$ to be the map

$$
\lambda_{1}\left(r_{1} \cdot e_{1}^{[1]} \otimes \varepsilon_{1}^{[1]}+r_{2} \cdot e_{1}^{[1]} \otimes \varepsilon_{1}^{[2]}+r_{3} \cdot e_{1}^{[2]} \otimes \varepsilon_{1}^{[1]}+r_{4} \cdot e_{1}^{[2]} \otimes \varepsilon_{1}^{[2]}\right)=-r_{1} \cdot \varepsilon_{2} .
$$

Define $\tau_{r}: \mathbb{L}_{r} \rightarrow \widehat{\mathbb{N}}_{r+1}$ by

$$
\begin{aligned}
& \tau_{-1}(1)^{(0)}(1 \otimes 1)=1 \otimes 1 \in \mathbb{F}_{0}(2)^{(0)} \\
& \tau_{0}(1)^{(0)}\left(1 \otimes \beta_{1}\right)=-1 \otimes \beta_{1} \in \mathbb{F}_{1}(2)^{(0)} \\
& \tau_{0}(1)^{(1)}\left(\beta_{1} \otimes 1\right)=1 \otimes \beta_{1} \in \mathbb{F}_{1}(3)^{(0)} \\
& \tau_{1}(1)^{(0)}\left(1 \otimes \alpha_{2}\right)=1 \otimes \alpha_{2} \in \mathbb{F}_{2}(2)^{(0)} \\
& \tau_{1}(1)^{(2)}\left(\alpha_{2} \otimes 1\right)=-1 \otimes \alpha_{2} \in \mathbb{F}_{2}(3)^{(0)} \\
& \tau_{2}(2)^{(2)}\left(e_{2} \otimes 1\right)=\left\{\begin{array}{c}
-\varepsilon_{2} \otimes 1 \\
\in \mathbb{F}_{3}(4)^{(2)} \\
+ \\
u \otimes \varepsilon_{2} \in \mathbb{F}_{3}(3)^{(1)} \\
+ \\
-\lambda_{1}\left[u \otimes u\left(\varepsilon_{2}\right)\right] \otimes \varepsilon_{2} \in \mathbb{F}_{3}(1)^{(2)}
\end{array}\right.
\end{aligned}
$$

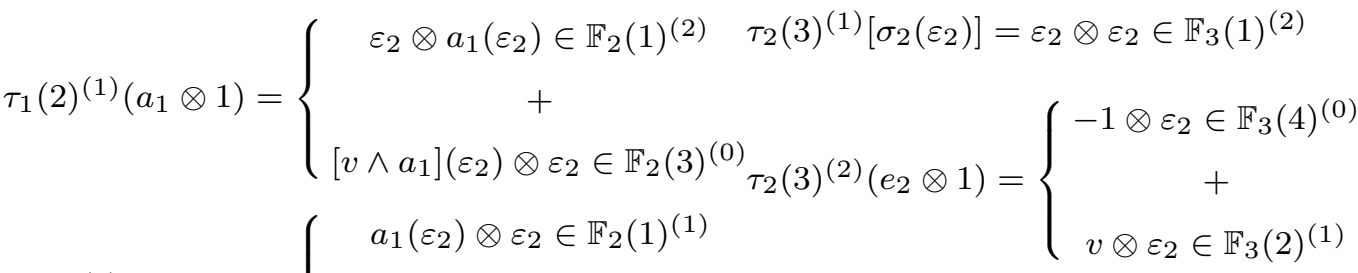

$$
\begin{aligned}
& \tau_{1}(3)^{(1)}\left(a_{1} \otimes 1\right)=\left\{\begin{array}{c}
a_{1}\left(\varepsilon_{2}\right) \otimes \varepsilon_{2} \in \mathbb{F}_{2}(1)^{(1)} \\
+\quad \begin{array}{c}
v \otimes \varepsilon_{2} \in \mathbb{F}_{3}(2)^{(1)} \\
{\left[a_{1} \wedge u\right]\left(\varepsilon_{2}\right) \otimes \varepsilon_{2} \in \mathbb{F}_{2}(2)^{(0)}}
\end{array} \tau_{2}(4)^{(0)}\left(1 \otimes \alpha_{1}\right)=\alpha_{1}\left(e_{2}\right) \otimes \varepsilon_{2} \in \mathbb{F}_{3}(2)^{(1)}
\end{array}\right. \\
& \left\{\begin{array}{c}
v_{1} \cdot \varepsilon_{2} \otimes \varepsilon_{1}^{[2]} \in \mathbb{F}_{2}(1)^{(2)} \\
+
\end{array} \quad \tau_{2}(4)^{(1)}\left(\alpha_{1} \otimes 1\right)=\left\{\begin{array}{c}
-\alpha_{1}\left(e_{2}\right) \otimes \varepsilon_{2} \in \mathbb{F}_{3}(3)^{(1)} \\
+ \\
\lambda_{1}\left[\alpha_{1}\left(e_{2}\right) \otimes u\left(\varepsilon_{2}\right)\right] \otimes \varepsilon_{2} \in \mathbb{F}_{3}(1)^{(2)}
\end{array}\right.\right. \\
& \tau_{1}(4)^{(0)}(1 \otimes 1)=\left\{v_{1} v_{2} \cdot 1 \otimes \varepsilon_{2} \in \mathbb{F}_{2}(3)^{(0)} \quad \tau_{3}(2)^{(2)}\left(e_{2} \otimes \alpha_{1}\right)=\varepsilon_{2} \otimes \alpha_{1} \in \mathbb{F}_{4}(4)^{(2)}\right. \\
& +\quad \tau_{3}(3)^{(2)}\left(e_{2} \otimes \alpha_{1}\right)=\alpha_{1} \otimes \varepsilon_{2} \in \mathbb{F}_{4}(4)^{(1)} \\
& \ell_{0}(1) \in\left[\mathbb{F}_{2}(2)^{(1)}\right]^{\prime \prime} \quad \tau_{4}(2)^{(2)}\left(e_{2} \otimes \varepsilon_{2}\right)=-\varepsilon_{2} \otimes \varepsilon_{2} \in \mathbb{F}_{5}(4)^{(2)} \text {. }
\end{aligned}
$$

Use Definition $3.12(\mathrm{~b})$ and (c) to define $\psi_{r}: \mathbb{F}_{r} \rightarrow \mathbb{M}_{r}$ and $m_{r}: \mathbb{M}_{r} \rightarrow \mathbb{M}_{r-1}$. It is not difficult to verify Lemma 3.13. Proposition 3.14 is a formal result; and therefore, it also holds. A direct calculation now shows that $\mathbb{M}$ is the Koszul complex on entries of $\operatorname{Adj} \mathbf{X}+\mathbf{v u}$. Indeed, if we let

$$
\begin{gathered}
g_{1}=x_{22}+v_{1} u_{1}, g_{2}=-x_{21}+v_{2} u_{1}, g_{3}=-x_{12}+v_{1} u_{2}, g_{4}=x_{11}+v_{2} u_{2} \text { in } R, \\
w_{1}=-\varepsilon_{1}^{[1]} \otimes \varepsilon_{1}^{[1]}, w_{2}=-\varepsilon_{1}^{[1]} \otimes \varepsilon_{1}^{[2]}, w_{3}=-\varepsilon_{1}^{[2]} \otimes \varepsilon_{1}^{[1]}, w_{4}=-\varepsilon_{1}^{[2]} \otimes \varepsilon_{1}^{[2]}, \text { in } \mathbb{F}_{1}(1)^{(1)}=\mathbb{M}_{1}, \\
w_{12}=e_{1}^{[2]} \otimes \varepsilon_{1}^{[1]} \in\left[\mathbb{F}_{2}(3)^{(1)}\right]^{\prime}, w_{13}=-e_{1}^{[2]} \otimes \varepsilon_{1}^{[1]} \in\left[\mathbb{F}_{2}(2)^{(1)}\right]^{\prime}, w_{14}=e_{1}^{[2]} \otimes \varepsilon_{1}^{[2]} \in\left[\mathbb{F}_{2}(3)^{(1)}\right]^{\prime}, \\
w_{23}=\left\{\begin{array}{c}
e_{1}^{[1]} \otimes \varepsilon_{1}^{[1]}-e_{1}^{[2]} \otimes \varepsilon_{1}^{[2]} \in\left[\mathbb{F}_{2}(2)^{(1)}\right]^{\prime} \\
+e_{1}^{[2]} \otimes \varepsilon_{1}^{[2]} \in\left[\mathbb{F}_{2}(3)^{(1)}\right]^{\prime}, \\
w_{24}=e_{1}^{[1]} \otimes \varepsilon_{1}^{[2]} \in\left[\mathbb{F}_{2}(2)^{(1)}\right]^{\prime}, w_{34}=-e_{1}^{[1]} \otimes \varepsilon_{1}^{[2]} \in\left[\mathbb{F}_{2}(3)^{(1)}\right]^{\prime} \text { in } \mathbb{M}_{2}, \\
w_{123}=\varepsilon_{1}^{[1]} \otimes \varepsilon_{1}^{[1]}, w_{124}=\varepsilon_{1}^{[1]} \otimes \varepsilon_{1}^{[2]}, w_{134}=-\varepsilon_{1}^{[2]} \otimes \varepsilon_{1}^{[1]}, w_{234}=-\varepsilon_{1}^{[2]} \otimes \varepsilon_{1}^{[2]} \text { in } \mathbb{F}_{3}(4)^{(1)}=\mathbb{M}_{3}, \text { and }
\end{array}\right.
\end{gathered}
$$


then we see that

$$
\begin{gathered}
m_{1}\left(w_{i}\right)=g_{i}, m_{2}\left(w_{i j}\right)=g_{i} \cdot w_{j}-g_{j} \cdot w_{i}, m_{3}\left(w_{i j k}\right)=g_{i} \cdot w_{j k}-g_{j} \cdot w_{i k}+g_{k} \cdot w_{i j}, \text { and } \\
m_{4}\left(w_{1234}\right)=g_{1} \cdot w_{234}-g_{2} \cdot w_{134}+g_{3} \cdot w_{124}-g_{4} \cdot w_{123}
\end{gathered}
$$

for all $i, j$, and $k$.

\section{Exactness.}

Theorem 4.1. Fix an integer $n$, with $2 \leq n$. Let $\mathbf{u}_{1 \times n}, \mathbf{X}_{n \times n}$, and $\mathbf{v}_{n \times 1}$ be matrices of indeterminates over a commutative noetherian ring $R_{0}, R$ be the polynomial ring $R_{0}\left[\left\{u_{i}, v_{i}, x_{i j} \mid 1 \leq i, j \leq n\right\}\right]$, and $u, X, v$ be the Data of 1.6 constructed from $\mathbf{u}, \mathbf{X}, \mathbf{v}$ by way of Convention 1.8. If $\mathbb{F}$ is the complex $\mathbb{F}[u, X, v]$ of Definition 2.1, then the homology $H_{i}(\mathbb{F})$ is zero for all integers $i$, except $i=0$.

Proof. The proof proceeds by induction on $n$. If $n=2$, then the result is established in Proposition 3.20. Henceforth, we assume that $3 \leq n$. The map

$$
f_{0}(2)^{(0)}: \mathbb{F}_{0}(2)^{(0)} \rightarrow \mathbb{F}_{-1}(1)^{(0)}
$$

is an isomorphism, and when this isomorphism is split from the complex $\mathbb{F}$, the resulting complex, $\overline{\mathbb{F}}$, has the same homology as $\mathbb{F}$ and looks like

$$
\overline{\mathbb{F}}: \quad 0 \rightarrow \overline{\mathbb{F}}_{2 n+1} \rightarrow \overline{\mathbb{F}}_{2 n} \rightarrow \cdots \rightarrow \overline{\mathbb{F}}_{0} \rightarrow 0 .
$$

Consequently, it suffices to apply the acyclicity lemma [5, Corollary 4.2] and prove that the homology of the localization $\mathbb{F}_{x}$ is concentrated in position zero for each fixed indeterminate $x=x_{i j}$. Let $R_{0}^{\prime}$ be the $\operatorname{ring} R_{0}\left[\left\{x_{i k}, x_{\ell j} \mid 1 \leq k \leq n, 1 \leq \ell \leq\right.\right.$ $\left.n, \ell \neq i\}, x^{-1}\right]$. It is easy to find matrices $M$ and $N$ with entries in $R_{0}^{\prime}$ such that

(a) $\operatorname{det} M=\operatorname{det} N=1$,

(b) $N \mathbf{X} M$ has the form $\left[\begin{array}{ll}1 & 0 \\ 0 & \mathbf{X}^{\prime}\end{array}\right]$,

(c) the entries of $X^{\prime}, M^{-1} \mathbf{v}, \mathbf{u} N^{-1}$ form a sequence of indeterminates $T_{1}, \ldots T_{m}$ over the ring $R_{0}^{\prime}$, where $m=(n-1)^{2}+2 n$, and

(d) the ring $R_{x}$ is equal to the polynomial ring $R_{0}^{\prime}\left[T_{1}, \ldots T_{m}\right]$.

Lemma 4.3 guarantees that $\mathbb{F}_{x}$ is isomorphic to the complex created using the data $\mathbf{u} N^{-1}, N \mathbf{X} M, M^{-1} \mathbf{v}$. Thus, $\mathbb{F}_{x}$ is isomorphic to the complex $(\mathbb{G}, g)$ of Lemma 4.11. In the notation of Lemma 4.11, $\mathbb{G}$ is "almost" the total complex of

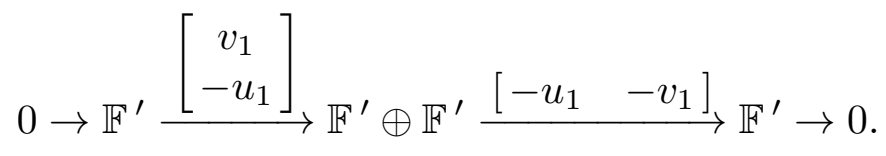

Indeed, $\mathbb{G}$ and the total complex of (4.2) differ only because the map $h_{r-2}$ in $g_{r}$ is not zero. Nonetheless, the induction hypothesis, applied to the generic data of $\mathbb{F}^{\prime}$, guarantees that the homology of $\mathbb{F}^{\prime}$ is concentrated in degree zero and that

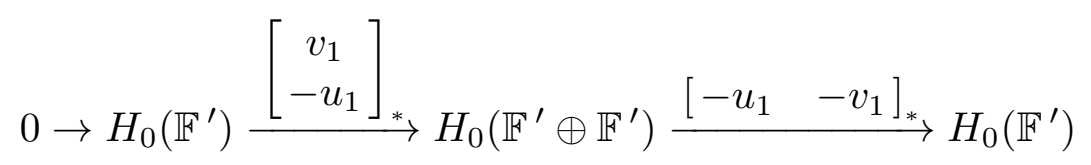

is an exact sequence. (Keep in mind that $u_{1}$ and $v_{1}$ are indeterminates over the polynomial ring obtained by adjoining the entries of of the matrices which represent $X^{\prime}, u^{\prime}$ and $v^{\prime}$ to $\left.R_{0}^{\prime}.\right)$ Lemma 4.7 now yields that $H_{k}\left(\mathbb{F}_{x}\right)=0$ for all $k \neq 0$. 
Lemma 4.3. Adopt Data 1.6. If $\theta: F \rightarrow F$ is an isomorphism with $\operatorname{det} \theta=1$, then the complexes

$$
\mathbb{F}\left[\theta^{-1}(u), \theta^{*} \circ X, v\right], \quad \mathbb{F}[u, X, v], \quad \text { and } \quad \mathbb{F}\left[u, X \circ \theta, \theta^{-1}(v)\right]
$$

are all isomorphic.

Proof. Let $\overline{\mathbb{F}}=\mathbb{F}\left[\theta^{-1}(u), \theta^{*} \circ X, v\right], \mathbb{F}=\mathbb{F}[u, X, v]$, and $\widetilde{\mathbb{F}}=\mathbb{F}\left[u, X \circ \theta, \theta^{-1}(v)\right]$. We define maps $\Theta: \mathbb{F} \rightarrow \widetilde{\mathbb{F}}$ and $\Phi: \mathbb{F} \rightarrow \overline{\mathbb{F}}$ by defining module isomorphisms

$$
\Theta_{r}(i)^{(t)}: \mathbb{F}_{r}(i)^{(t)} \rightarrow \widetilde{\mathbb{F}}_{r}(i)^{(t)} \quad \text { and } \quad \Phi_{r}(i)^{(t)}: \mathbb{F}_{r}(i)^{(t)} \rightarrow \overline{\mathbb{F}}_{r}(i)^{(t)}
$$

for all $i, r$, and $t$, as follows:

$$
\begin{aligned}
& \Theta_{r}(1)^{(t)}\left(\alpha_{t} \otimes \beta_{s}\right)=\alpha_{t} \otimes\left(\bigwedge^{s} \theta^{*}\right)\left(\beta_{s}\right), \quad \Phi_{r}(1)^{(t)}\left(\alpha_{t} \otimes \beta_{s}\right)=\left(\bigwedge^{t} \theta^{*}\right)\left(\alpha_{t}\right) \otimes \beta_{s}, \\
& \Theta_{r}(2)^{(t)}\left(a_{t} \otimes \beta_{s}\right)=\left(\bigwedge^{t} \theta^{-1}\right)\left(a_{t}\right) \otimes\left(\bigwedge^{s} \theta^{*}\right)\left(\beta_{s}\right), \quad \Phi_{r}(2)^{(t)}\left(a_{t} \otimes \beta_{s}\right)=a_{t} \otimes \beta_{s}, \\
& \Theta_{r}(3)^{(t)}\left(a_{t} \otimes \beta_{s}\right)=a_{t} \otimes \beta_{s}, \quad \Phi_{r}(3)^{(t)}\left(a_{t} \otimes \beta_{s}\right)=\left(\bigwedge^{t} \theta^{-1}\right)\left(a_{t}\right) \otimes\left(\bigwedge^{s} \theta^{*}\right)\left(\beta_{s}\right), \\
& \Theta_{r}(4)^{(t)}\left(\alpha_{t} \otimes \beta_{s}\right)=\alpha_{t} \otimes\left(\bigwedge^{s} \theta^{*}\right)\left(\beta_{s}\right) \text {, and } \quad \Phi_{r}(4)^{(t)}\left(\alpha_{t} \otimes \beta_{s}\right)=\left(\bigwedge^{t} \theta^{*}\right)\left(\alpha_{t}\right) \otimes \beta_{s} \text {. }
\end{aligned}
$$

A direct calculation shows that $\Theta$ and $\Phi$ are maps of complexes; the following identities are used:

$$
(4.6)\left(\bigwedge^{t-1} \theta^{-1}\right)\left(\left[\beta_{1} \wedge\left(\bigwedge^{n-t} X^{*}\right)\left(\alpha_{t}\left[e_{n}\right]\right)\right]\left(e_{n}\right)\right)=\left[\theta^{*}\left(\beta_{1}\right) \wedge\left[\wedge^{n-t}(X \circ \theta)^{*}\right]\left(\alpha_{t}\left[e_{n}\right]\right)\right]\left(e_{n}\right) .
$$

Identity (4.4) is obvious. To prove (4.5), apply Observation 1.11 (a) to see that the left side is equal to

$$
\left(\bigwedge^{n-s} X\right)\left(\beta_{s}\left[\left(\bigwedge^{n} \theta\right)\left[e_{n}\right]\right]\right)
$$

The hypothesis $\operatorname{det} \theta=1$ ensures that $\left(\bigwedge^{n} \theta\right)\left[e_{n}\right]=e_{n}$. The right side of (4.6) is equal to $\left(\left(\bigwedge^{n-t+1} \theta^{*}\right)\left[\beta_{1} \wedge\left(\bigwedge^{n-t} X^{*}\right)\left(\alpha_{t}\left[e_{n}\right]\right)\right]\right)\left(e_{n}\right)$. Apply the isomorphism $\bigwedge^{t-1} \theta$ to both sides of the proposed identity and use Observation 1.11 (a), as well as the hypothesis $\operatorname{det} \theta=1$, to complete the proof of (4.6).

Lemma 4.7. Let $(\mathbb{T}, t) \stackrel{\alpha}{\rightarrow}(\mathbb{M}, m) \stackrel{\beta}{\rightarrow}(\mathbb{B}, b)$ be a complex of complexes over the commutative noetherian ring $R,\left\{h_{i}: \mathbb{T}_{i} \rightarrow \mathbb{B}_{i+1}\right\}$ be a family of maps which satisfies

$$
b_{i+1} \circ h_{i}+h_{i-1} \circ t_{i}=0 \text { for all } i,
$$

and $(\mathbb{D}, d)$ be the complex with $\mathbb{D}_{i}=\mathbb{B}_{i} \oplus \mathbb{M}_{i-1} \oplus \mathbb{T}_{i-2}$ and

$$
d_{i}=\left[\begin{array}{ccc}
b_{i} & (-1)^{i-1} \beta_{i-1} & h_{i-2} \\
0 & m_{i-1} & (-1)^{i} \alpha_{i-2} \\
0 & 0 & t_{i-2}
\end{array}\right]
$$




$$
0 \rightarrow H_{0}(\mathbb{T}) \stackrel{\alpha_{*}}{\longrightarrow} H_{0}(\mathbb{M}) \stackrel{\beta_{*}}{\longrightarrow} H_{0}(\mathbb{B})
$$

$i s$ an exact sequence of $R$-modules, then $H_{i}(\mathbb{D})=0$ for all $i \neq 0$.

Proof. Let $(\mathbb{C}, c)$ be the mapping cone of the $\alpha: \mathbb{T} \rightarrow \mathbb{M}$. In other words, $\mathbb{C}_{i}=$ $\mathbb{M}_{i} \oplus \mathbb{T}_{i-1}$ and

$$
c_{i}=\left[\begin{array}{cc}
m_{i} & (-1)^{i-1} \alpha_{i-1} \\
0 & t_{i-1}
\end{array}\right] .
$$

The long exact sequence of homology, which is associated to a mapping cone, yields the exact sequence

$$
0 \rightarrow H_{0}(\mathbb{T}) \stackrel{\alpha_{*}}{\longrightarrow} H_{0}(\mathbb{M}) \rightarrow H_{0}(\mathbb{C}) \rightarrow 0
$$

as well as $H_{i}(\mathbb{C})=0$ for $i \neq 0$. Observe that $\mathbb{D}$ is the mapping cone of $[\beta h]: \mathbb{C} \rightarrow \mathbb{B}$. (The map $\mathbb{C}_{i} \rightarrow \mathbb{B}_{i}$ is given by $\left[\beta_{i}(-1)^{i} h_{i-1}\right]$.) Let $\mathbb{D}^{\prime}$ be the mapping cone of $\left[\begin{array}{ll}\beta & 0\end{array}\right]: \mathbb{C} \rightarrow \mathbb{B}$. The long exact sequence of homology gives exact sequences

$$
\begin{aligned}
& 0 \rightarrow H_{1}\left(\mathbb{D}^{\prime}\right) \rightarrow \mathrm{H}_{0}(\mathbb{C}) \stackrel{[\beta}{[\beta]_{*}} \longrightarrow H_{0}(\mathbb{B}) \rightarrow H_{0}\left(\mathbb{D}^{\prime}\right) \rightarrow 0, \text { and } \\
& 0 \rightarrow H_{1}(\mathbb{D}) \rightarrow \mathrm{H}_{0}(\mathbb{C}) \stackrel{[\beta]_{*}}{\longrightarrow} H_{0}(\mathbb{B}) \rightarrow H_{0}(\mathbb{D}) \rightarrow 0,
\end{aligned}
$$

as well as $H_{i}(\mathbb{D})=H_{i}\left(\mathbb{D}^{\prime}\right)=0$ for $i \neq 0$ or 1 . The hypothesis ensures that $\left[\begin{array}{ll}\beta & 0\end{array}\right]_{*}$ is an injection. The proof is complete because $[\beta 0]_{*}$ and $[\beta h]_{*}$ are the same map from $\mathrm{H}_{0}(\mathbb{C})$ to $\mathrm{H}_{0}(\mathbb{B})$. Indeed, if $\left[\begin{array}{c}z_{0} \\ z_{-1}\end{array}\right]$ is a cycle in $\mathbb{C}_{0}$, then $t_{-1}\left(z_{-1}\right)=0$ and $z_{-1}=t_{0}\left(y_{0}\right)$ for some $y_{0}$ in $\mathbb{T}_{0}$. It follows that

$$
h_{-1}\left(z_{-1}\right)=h_{-1} \circ t_{0}\left(y_{0}\right)=-b_{1} \circ h_{0}\left(y_{0}\right) \in \operatorname{im} b_{1} .
$$

Remark 4.8. Adopt Data 1.6. For each pair of integers $(i, j)$, let $f_{r}[i, j]$ be the composition

$$
\mathbb{F}_{r}(j) \stackrel{\text { incl }}{\longrightarrow} \mathbb{F}_{r} \stackrel{f_{r}}{\longrightarrow} \mathbb{F}_{r-1} \stackrel{\text { proj }}{\longrightarrow} \mathbb{F}_{r-1}(i) .
$$

Notice that the map $f_{r}: \mathbb{F}_{r} \rightarrow \mathbb{F}_{r-1}$ is given by

$$
f_{r}=\left[\begin{array}{cccc}
f_{r}[1,1] & f_{r}[1,2] & f_{r}[1,3] & 0 \\
f_{r}[2,1] & f_{r}[2,2] & 0 & f_{r}[2,4] \\
f_{r}[3,1] & 0 & f_{r}[3,3] & f_{r}[3,4] \\
0 & f_{r}[4,2] & f_{r}[4,3] & f_{r}[4,4]
\end{array}\right]
$$

Define a new map $\tilde{f}_{r}: \mathbb{F}_{r} \rightarrow \mathbb{F}_{r-1}$ by

$$
\widetilde{f}_{r}=\left[\begin{array}{cccc}
f_{r}[1,1] & f_{r}[1,2] & f_{r}[1,3] & 0 \\
-f_{r}[2,1] & f_{r}[2,2] & 0 & -f_{r}[2,4] \\
-f_{r}[3,1] & 0 & f_{r}[3,3] & -f_{r}[3,4] \\
0 & f_{r}[4,2] & f_{r}[4,3] & f_{r}[4,4]
\end{array}\right] .
$$

Each map $f_{\bullet}[i, i]$ is a Koszul map; and therefore, $f_{r}[i, i] \circ f_{r+1}[i, i]=0$. A quick calculation now shows that

$$
\ldots \stackrel{\widetilde{f}_{r+1}}{\longrightarrow} \mathbb{F}_{r} \stackrel{\widetilde{f}_{r}}{\longrightarrow} \mathbb{F}_{r-1} \stackrel{\widetilde{f}_{r-1}}{\longrightarrow} \ldots
$$


Lemma 4.10. If the data of 1.6 is adopted, then the complex $\mathbb{F}[u, X,-v]$ of Definition 2.1 is isomorphic to the complex $\widetilde{\mathbb{F}}[u, X, v]$ of $(4.9)$.

Proof. For integers $i, r$, and $t$ define the module isomorphism

$$
\begin{gathered}
\theta_{r}(i)^{(t)}: \mathbb{F}_{r}(i)^{t} \rightarrow \mathbb{F}_{r}(i)^{(t)} \text { by } \\
\theta_{r}(1)^{(t)}=(-1)^{r+1-t} \mathrm{id}, \theta_{r}(2)^{(t)}=(-1)^{r-t} \mathrm{id}, \theta_{r}(3)^{(t)}=(-1)^{t} \mathrm{id}, \text { and } \theta_{r}(4)^{(t)}=(-1)^{r-t} \mathrm{id} .
\end{gathered}
$$

It is not difficult to see that $\theta: \widetilde{\mathbb{F}}[u, X, v] \rightarrow \mathbb{F}[u, X,-v]$ is a homomorphism of complexes.

Most of the statement of the next result consists of notation. The only hypothesis is labeled (4.12).

Lemma 4.11. Adopt Data 1.6. Let $R e \oplus F^{\prime}$ be a decomposition of $F$ into a direct sum of two free summands, and let $R \varepsilon \oplus F^{\prime *}$ be the corresponding decomposition of $F^{*}$. Let $u_{1}$ and $v_{1}$ be elements of $R$ and $u^{\prime}$ and $v^{\prime}$ be elements of $F^{\prime}$ with $u=u_{1} e+u^{\prime}$ and $v=v_{1} e+v^{\prime}$. Let $e_{n-1}^{\prime}$ be the orientation element of $\bigwedge^{n-1} F^{\prime}$ which has the property that $e_{n}=e \wedge e_{n-1}^{\prime}$. Suppose that $X$ may be decomposed as

$$
X=\left[\begin{array}{cc}
1 & 0 \\
0 & X^{\prime}
\end{array}\right],
$$

for some map $X^{\prime}: F^{\prime} \rightarrow F^{\prime}$. Let $(\mathbb{F}, f)$ be the complex $\mathbb{F}[u, X, v]$ and $\left(\mathbb{F}^{\prime}, f^{\prime}\right)$ be the complex $\mathbb{F}\left[u^{\prime}, X^{\prime},(-1)^{n-1} v^{\prime}\right]$. Then there are homomorphisms $h_{r}: \mathbb{F}_{r}^{\prime} \rightarrow \mathbb{F}_{r+1}^{\prime}$ such that the complexes $(\mathbb{F}, f)$ and $(\mathbb{G}, g)$ are isomorphic, where

$$
\mathbb{G}_{r}=\mathbb{F}_{r}^{\prime} \oplus \mathbb{F}_{r-1}^{\prime} \oplus \mathbb{F}_{r-1}^{\prime} \oplus \mathbb{F}_{r-2}^{\prime} \quad \text { and } \quad g_{r}=\left[\begin{array}{cccc}
f_{r}^{\prime} & (-1)^{r} u_{1} & (-1)^{r} v_{1} & h_{r-2} \\
0 & f_{r-1}^{\prime} & 0 & (-1)^{r} v_{1} \\
0 & 0 & f_{r-1}^{\prime} & (-1)^{r+1} u_{1} \\
0 & 0 & 0 & f_{r-2}^{\prime}
\end{array}\right] \text {. }
$$

Proof. We have chosen to let $\left(\mathbb{F}^{\prime}, f^{\prime}\right)$ represent the complex $\mathbb{F}\left[u^{\prime}, X^{\prime},(-1)^{n-1} v^{\prime}\right]$ because this choice of notation leads to a clean statement of the result; however, all of our calculations are made using the complex $\mathbb{F}\left[u^{\prime}, X^{\prime}, v^{\prime}\right]$, which we refer to as $\left(\mathbb{F}^{\prime}, f^{\prime \prime}\right)$. The maneuver from $\left(\mathbb{F}^{\prime}, f^{\prime \prime}\right)$ to $\left(\mathbb{F}^{\prime}, f^{\prime}\right)$ uses Lemma 4.10 , and occurs at the end of the proof.

For each integer $r$, let $h_{r}^{\prime}: \mathbb{F}_{r}^{\prime} \rightarrow \mathbb{F}_{r+1}^{\prime}$ be the homomorphism which is given by

$$
\begin{aligned}
& h_{r}^{\prime}(1)^{(t)}\left(\alpha_{t}^{\prime} \otimes \beta_{s}^{\prime}\right)=\left\{\begin{array}{c}
(-1)^{n-1+r}\left[\left(\bigwedge^{n-1-t} X^{\prime *}\right)\left(\alpha_{t}^{\prime}\left[e_{n-1}^{\prime}\right]\right)\right]\left(e_{n-1}^{\prime}\right) \otimes \beta_{s}^{\prime} \in \mathbb{F}_{r+1}^{\prime}(2)^{(t)} \\
+ \\
(-1)^{n+1+r}\left[\left(\bigwedge^{n-1-s} X^{\prime}\right)\left(\beta_{s}^{\prime}\left[e_{n-1}^{\prime}\right]\right)\right]\left(e_{n-1}^{\prime}\right) \otimes \alpha_{t}^{\prime} \in \mathbb{F}_{r+1}^{\prime}(3)^{(s)},
\end{array}\right. \\
& h_{r}^{\prime}(2)^{(t)}\left(a_{t}^{\prime} \otimes \beta_{s}^{\prime}\right)=(-1)^{r-1}\left(\bigwedge^{t} X^{\prime}\right)\left(a_{t}^{\prime}\right) \otimes \beta_{s}^{\prime} \in \mathbb{F}_{r+1}^{\prime}(4)^{(t)}, \\
& h_{r}^{\prime}(3)^{(t)}\left(a_{t}^{\prime} \otimes \beta_{s}^{\prime}\right)=(-1)^{r} \beta_{s}^{\prime} \otimes\left(\bigwedge^{t} X^{\prime *}\right)\left(a_{t}^{\prime}\right) \in \mathbb{F}_{r+1}^{\prime}(4)^{(s)}, \text { and } \\
& h_{r}^{\prime}(4)=0 .
\end{aligned}
$$

The direct sum decompositions of $F$ and $F^{*}$ give rise to decompositions

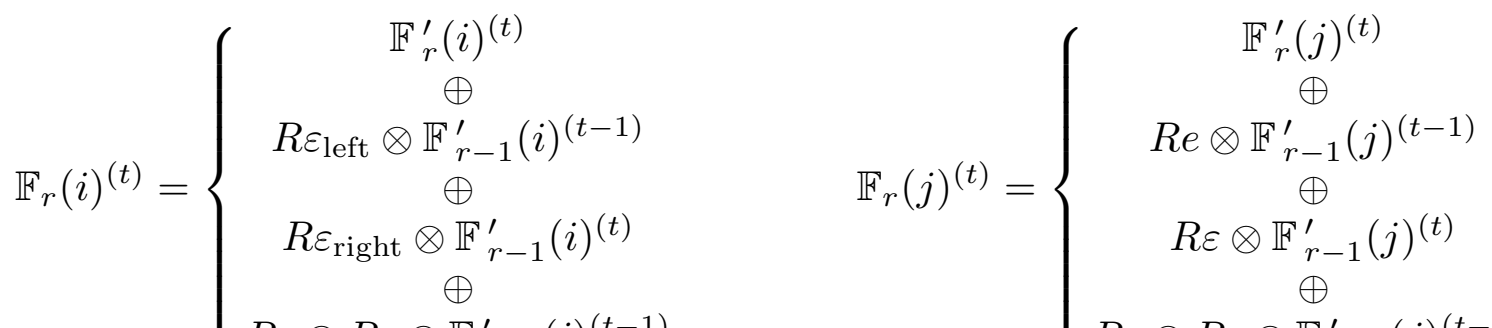


for $i=1,4$ and $j=2,3$. The notation is self-explanatory; for example, if $t+s=$ $r+1$, then

$\mathbb{F}_{r}(1)^{(t)}=\bigwedge^{t} F^{*} \otimes \bigwedge^{s} F^{*}=\left(\bigwedge^{t} F^{\prime *} \oplus\left[R \varepsilon \otimes \bigwedge^{t-1} F^{* *}\right]\right) \otimes\left(\bigwedge^{s} F^{\prime *} \oplus\left[R \varepsilon \otimes \bigwedge^{s-1} F^{*}\right]\right)$

and we let $R \varepsilon_{\text {left }} \otimes \mathbb{F}_{r-1}^{\prime}(1)^{(t-1)}$ represent the summand $\left[R \varepsilon \otimes \bigwedge^{t-1} F^{\prime *}\right] \otimes \bigwedge^{s} F^{\prime *}$ of $\mathbb{F}_{r}(1)^{(t)}$. Now that $\mathbb{F}_{r}$ has been decomposed into 16 summands, we recombine the pieces. Let

$$
\begin{gathered}
\mathbb{A}_{r}=\mathbb{F}_{r}^{\prime}(1) \oplus \mathbb{F}_{r}^{\prime}(2) \oplus \mathbb{F}_{r}^{\prime}(3) \oplus \mathbb{F}_{r}^{\prime}(4), \\
\mathbb{B}_{r}=\left(R \varepsilon_{\text {left }} \otimes \mathbb{F}_{r-1}^{\prime}(1)\right) \oplus\left(R e \otimes \mathbb{F}_{r-1}^{\prime}(2)\right) \oplus\left(R \varepsilon \otimes \mathbb{F}_{r-1}^{\prime}(3)\right) \oplus\left(R \varepsilon_{\text {left }} \otimes \mathbb{F}_{r-1}^{\prime}(4)\right), \\
\mathbb{C}_{r}=\left(R \varepsilon_{\text {right }} \otimes \mathbb{F}_{r-1}^{\prime}(1)\right) \oplus\left(R \varepsilon \otimes \mathbb{F}_{r-1}^{\prime}(2)\right) \oplus\left(R e \otimes \mathbb{F}_{r-1}^{\prime}(3)\right) \oplus\left(R \varepsilon_{\text {right }} \otimes \mathbb{F}_{r-1}^{\prime}(4)\right),
\end{gathered}
$$

and

$\mathbb{D}_{r}=\left(R \varepsilon \otimes R \varepsilon \otimes \mathbb{F}_{r-2}^{\prime}(1)\right) \oplus\left(R e \otimes R \varepsilon \otimes \mathbb{F}_{r-2}^{\prime}(2)\right) \oplus\left(R e \otimes R \varepsilon \otimes \mathbb{F}_{r-2}^{\prime}(3)\right) \oplus\left(R \varepsilon \otimes R \varepsilon \otimes \mathbb{F}_{r-2}(4)\right)$.

Observe that $\mathbb{F}_{r}=\mathbb{A}_{r} \oplus \mathbb{B}_{r} \oplus \mathbb{C}_{r} \oplus \mathbb{D}_{r}$.

We establish the isomorphism $(\mathbb{F}, f) \cong(\mathbb{G}, g)$ in two steps. First we create a complex $(\widehat{\mathbb{F}}, \widehat{f})$ and establish an isomorphism from $(\widehat{\mathbb{F}}, \widehat{f})$ to $(\mathbb{F}, f)$. Then, we prove that $(\widehat{\mathbb{F}}, \widehat{f})$ is isomorphic to $(\mathbb{G}, g)$.

For each pair of integers $(i, j)$, let $f_{r}^{\prime \prime}[i, j]: \mathbb{F}_{r}^{\prime}(j) \rightarrow \mathbb{F}_{r}^{\prime}(i)$ be the map which is described in Remark 4.8. Let $\mathfrak{f}_{r}: \mathbb{F}_{r}^{\prime} \rightarrow \mathbb{F}_{r-1}^{\prime}$ be the map which is given by

$$
\mathfrak{f}_{r}=\left[\begin{array}{cccc}
f_{r}^{\prime \prime}[1,1] & f_{r}^{\prime \prime}[1,2] & f_{r}^{\prime \prime}[1,3] & 0 \\
(-1)^{n-1} f_{r}^{\prime \prime}[2,1] & f_{r}^{\prime \prime}[2,2] & 0 & (-1)^{n-1} f_{r}^{\prime \prime}[2,4] \\
(-1)^{n-1} f_{r}^{\prime \prime}[3,1] & 0 & f_{r}^{\prime \prime}[3,3] & (-1)^{n-1} f_{r}^{\prime \prime}[3,4] \\
0 & f_{r}^{\prime \prime}[4,2] & f_{r}^{\prime \prime}[4,3] & f_{r}^{\prime \prime}[4,4]
\end{array}\right]
$$

We notice that

$$
\mathfrak{f}_{r}= \begin{cases}f_{r}^{\prime \prime}, & \text { if } n \text { is odd, and } \\ \widetilde{f^{\prime \prime}}{ }_{r}, & (\text { in the sense of Remark 4.8), if } n \text { is even. }\end{cases}
$$

In any event, Remark 4.8 shows that $\left(\mathbb{F}^{\prime}, \mathfrak{f}\right)$ is a complex. We now define the complex $(\widehat{\mathbb{F}}, \widehat{f})$ by $\widehat{\mathbb{F}}_{r}=\mathbb{G}_{r}$, and

$$
\widehat{f}_{r}=\left[\begin{array}{cccc}
\mathfrak{f}_{r} & (-1)^{r} u_{1} & (-1)^{r} v_{1} & h_{r-2}^{\prime} \\
0 & \mathfrak{f}_{r-1} & 0 & (-1)^{r} v_{1} \\
0 & 0 & \mathfrak{f}_{r-1} & (-1)^{r+1} u_{1} \\
0 & 0 & 0 & \mathfrak{f}_{r-2}
\end{array}\right]
$$

We find it convenient to give an additional name to the components of

$$
\widehat{\mathbb{F}}_{r}=\mathbb{F}_{r}^{\prime} \oplus \mathbb{F}_{r-1}^{\prime} \oplus \mathbb{F}_{r-1}^{\prime} \oplus \mathbb{F}_{r-2}^{\prime}
$$

Let $\widehat{\mathbb{A}}_{r}, \widehat{\mathbb{B}}_{r}, \widehat{\mathbb{C}}_{r}$, and $\widehat{\mathbb{D}}_{r}$ represent $\mathbb{F}_{r}^{\prime}$, the first $\mathbb{F}_{r-1}^{\prime}$, the second $\mathbb{F}_{r-1}^{\prime}$, and $\mathbb{F}_{r-2}^{\prime}$, respectively. There are natural isomorphisms 
for example, if $a_{t}^{\prime} \otimes \beta_{s}^{\prime} \in \mathbb{F}_{r-1}^{\prime}(3)^{(t)} \subseteq \widehat{\mathbb{B}}_{r}$, then

$$
\operatorname{nat}\left(a_{t}^{\prime} \otimes \beta_{s}^{\prime}\right)=a_{t}^{\prime} \otimes \varepsilon \wedge \beta_{s}^{\prime} \in R \varepsilon \otimes \mathbb{F}_{r-1}^{\prime}(3)^{(t)} \subseteq \mathbb{B}_{r}
$$

Let $\rho_{r}: \mathbb{F}_{r}^{\prime} \rightarrow \mathbb{F}_{r}^{\prime}$ be the isomorphism

$$
\rho_{r}=\left[\begin{array}{cccc}
1 & 0 & 0 & 0 \\
0 & -1 & 0 & 0 \\
0 & 0 & -1 & 0 \\
0 & 0 & 0 & 1
\end{array}\right]:\left\{\begin{array} { c } 
{ \mathbb { F } _ { r } ^ { \prime } ( 1 ) } \\
{ \oplus } \\
{ \mathbb { F } _ { r } ^ { \prime } ( 2 ) } \\
{ \oplus } \\
{ \mathbb { F } _ { r } ^ { \prime } ( 3 ) } \\
{ \oplus } \\
{ \mathbb { F } _ { r } ^ { \prime } ( 4 ) }
\end{array} \longrightarrow \left\{\begin{array}{c}
\mathbb{F}_{r}^{\prime}(1) \\
\oplus \\
\mathbb{F}_{r}^{\prime}(2) \\
\oplus \\
\mathbb{F}_{r}^{\prime}(3) \\
\oplus \\
\mathbb{F}_{r}^{\prime}(4) .
\end{array}\right.\right.
$$

For each integer $r$, consider the module isomorphism $\varphi_{r}: \widehat{\mathbb{F}}_{r} \rightarrow \mathbb{F}_{r}$, which is given by

$$
\begin{array}{ll}
\widehat{\mathbb{A}}_{r}=\mathbb{F}_{r}^{\prime} \stackrel{\text { id }}{\longrightarrow} \mathbb{A}_{r}, & \widehat{\mathbb{B}}_{r}=\mathbb{F}_{r-1}^{\prime} \stackrel{\rho_{r-1}}{\longrightarrow} \mathbb{F}_{r-1}^{\prime} \stackrel{\text { nat }}{\longrightarrow} \mathbb{B}_{r}, \\
\widehat{\mathbb{C}}_{r}=\mathbb{F}_{r-1}^{\prime} \stackrel{(-1)^{r}}{\longrightarrow} \mathbb{F}_{r-1}^{\prime} \stackrel{\text { nat }}{\longrightarrow} \mathbb{C}_{r}, \text { and } & \widehat{\mathbb{D}}_{r}=\mathbb{F}_{r-2}^{\prime} \stackrel{(-1)^{r} \cdot \rho_{r-2}}{\longrightarrow} \mathbb{F}_{r-2}^{\prime} \stackrel{\text { nat }}{\longrightarrow} \mathbb{D}_{r} .
\end{array}
$$

A very long, but straightforward, calculation yields that

$$
\varphi:(\widehat{\mathbb{F}}, \widehat{f}) \rightarrow(\mathbb{F}, f) \text { is a homomorphism of complexes. }
$$

Assume, for the time being, that (4.13) is established. If $n$ is odd, then we take $h_{r}=h_{r}^{\prime}$. In this case, $(\widehat{\mathbb{F}}, \widehat{f})$ is already equal to $(\mathbb{G}, g)$ and the proof is complete. If $n$ is even, then let $\theta:\left(\mathbb{F}^{\prime}, \mathfrak{f}\right) \rightarrow\left(\mathbb{F}^{\prime}, f^{\prime}\right)$ be the isomorphism of Lemma 4.10 and let $h_{r}=\theta_{r+1} \circ h_{r}^{\prime} \circ \theta_{r}^{-1}$. It is not difficult to see that the isomorphism

$$
\left[\begin{array}{cccc}
\theta_{r} & 0 & 0 & 0 \\
0 & \theta_{r-1} & 0 & 0 \\
0 & 0 & \theta_{r-1} & 0 \\
0 & 0 & 0 & \theta_{r-2}
\end{array}\right]: \widehat{\mathbb{F}}_{r} \rightarrow \mathbb{G}_{r}
$$

induces an isomorphism of complexes from $(\widehat{\mathbb{F}}, \widehat{f})$ to $(\mathbb{G}, g)$. Once again, the proof is complete.

Now, we turn our attention to proving (4.13). The proof involves sixteen calculations. We record four of these calculations and suppress the remaining twelve. No new ideas are required for the suppressed calculations. Fix integers $r, s$, and $t$ with $t+s=r+1$. We begin with $y=\alpha_{t}^{\prime} \otimes \beta_{s}^{\prime} \in \mathbb{F}_{r}^{\prime}(1)^{(t)} \subseteq \widehat{\mathbb{A}}_{r}$. Let $\Delta\left(\alpha_{t}^{\prime}\right)=\sum_{j} \alpha_{1}^{\prime[j]} \otimes \alpha_{t-1}^{\prime[j]}$ and $\Delta\left(\beta_{s}^{\prime}\right)=\sum_{i} \beta_{1}^{\prime[i]} \otimes \beta_{s-1}^{\prime[i]}$. We show that

$$
f_{r} \circ \varphi_{r}(y)=\varphi_{r-1} \circ \widehat{f}_{r}(y) .
$$

The left side of (4.14) is equal to

$$
f_{r}(1)^{(t)}\left(\alpha_{t}^{\prime} \otimes \beta_{s}^{\prime}\right)=\left\{\begin{array}{c}
\alpha_{t}^{\prime} \otimes v\left(\beta_{s}^{\prime}\right) \in \mathbb{F}_{r-1}(1)^{(t)} \\
+ \\
(-1)^{r} u\left(\alpha_{t}^{\prime}\right) \otimes \beta_{s}^{\prime} \in \mathbb{F}_{r-1}(1)^{(t-1)} \\
+ \\
\sum_{i}\left[\beta_{1}^{\prime[i]} \wedge\left(\bigwedge^{n-t} X^{*}\right)\left(\alpha_{t}^{\prime}\left[e_{n}\right]\right)\right]\left(e_{n}\right) \otimes \beta_{s-1}^{\prime[i]} \in \mathbb{F}_{r-1}(2)^{(t-1)} \\
\sum_{+}\left[\alpha_{1}^{\prime[j]} \wedge\left(\bigwedge^{n-s} X\right)\left(\beta_{s}^{\prime}\left[e_{n}\right]\right)\right]\left(e_{n}\right) \otimes \alpha_{t-1}^{\prime[j]} \in \mathbb{F}_{r-1}(3)^{(s-1)}
\end{array}\right.
$$


It is clear that $v\left(\beta_{s}^{\prime}\right)=v^{\prime}\left(\beta_{s}^{\prime}\right)$ and $u\left(\alpha_{t}^{\prime}\right)=u^{\prime}\left(\alpha_{t}^{\prime}\right)$. Use Proposition 1.2 (a) to see that

$$
\alpha_{t}^{\prime}\left(e_{n}\right)=(-1)^{t} e \wedge \alpha_{t}^{\prime}\left(e_{n-1}^{\prime}\right)
$$

It follows that

$$
\begin{aligned}
{\left[\beta_{1}^{\prime[i]} \wedge\left(\bigwedge^{n-t} X^{*}\right)\left(\alpha_{t}^{\prime}\left[e_{n}\right]\right)\right]\left(e_{n}\right) } & =(-1)^{t}\left[\beta_{1}^{\prime[i]} \wedge X(e) \wedge\left(\bigwedge^{n-t-1} X^{\prime *}\right)\left(\alpha_{t}^{\prime}\left[e_{n-1}^{\prime}\right]\right)\right]\left(e_{n}\right) \\
& =(-1)^{n-1}\left[\beta_{1}^{\prime[i]} \wedge\left(\bigwedge^{n-t-1} X^{\prime *}\right)\left(\alpha_{t}^{\prime}\left[e_{n-1}^{\prime}\right]\right)\right]\left(e_{n-1}^{\prime}\right)
\end{aligned}
$$

and we see that the left side of (4.14) is equal to

$$
\left\{\begin{array}{c}
\alpha_{t}^{\prime} \otimes v^{\prime}\left(\beta_{s}^{\prime}\right) \in \mathbb{F}_{r-1}^{\prime}(1)^{(t)} \\
+ \\
(-1)^{r} u^{\prime}\left(\alpha_{t}^{\prime}\right) \otimes \beta_{s}^{\prime} \in \mathbb{F}_{r-1}^{\prime}(1)^{(t-1)} \\
+ \\
(-1)^{n-1} \sum_{i}\left[\beta_{1}^{\prime[i]} \wedge\left(\bigwedge^{n-t-1} X^{\prime *}\right)\left(\alpha_{t}^{\prime}\left[e_{n-1}^{\prime}\right]\right)\right]\left(e_{n-1}^{\prime}\right) \otimes \beta_{s-1}^{\prime[i]} \in \mathbb{F}_{r-1}^{\prime}(2)^{(t-1)} \\
+ \\
(-1)^{n-1} \sum_{j}\left[\alpha_{1}^{\prime[j]} \wedge\left(\bigwedge^{n-s-1} X^{\prime}\right)\left(\beta_{s}^{\prime}\left[e_{n-1}^{\prime}\right]\right)\right]\left(e_{n-1}^{\prime}\right) \otimes \alpha_{t-1}^{\prime[j]} \in \mathbb{F}_{r-1}^{\prime}(3)^{(s-1)}
\end{array}\right.
$$

which is the same as the right side of (4.14). In our second calculation, we take $y$ equal to $\alpha_{t-1}^{\prime} \otimes \beta_{s}^{\prime} \in \mathbb{F}_{r-1}^{\prime}(1)^{(t-1)} \subseteq \widehat{\mathbb{B}}_{r}$. Let $\Delta\left(\alpha_{t-1}^{\prime}\right)=\sum_{j} \alpha_{1}^{\prime[j]} \otimes \alpha_{t-2}^{\prime[j]}$, and $\Delta\left(\beta_{s}^{\prime}\right)=\sum_{i} \beta_{1}^{\prime[i]} \otimes \beta_{s-1}^{\prime[i]}$. The left side of (4.14) is equal to

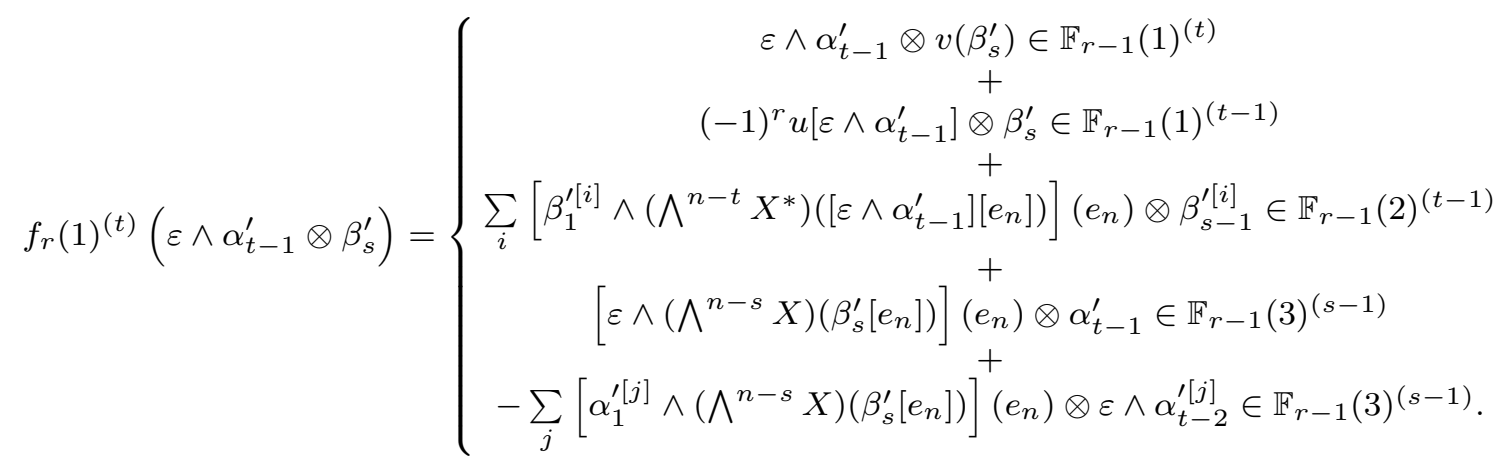

Use (4.15) to see that $\varepsilon \wedge\left(\bigwedge^{n-s} X\right)\left(\beta_{s}^{\prime}\left[e_{n}\right]\right)=0$. It follows that the left side of (4.14) is equal to

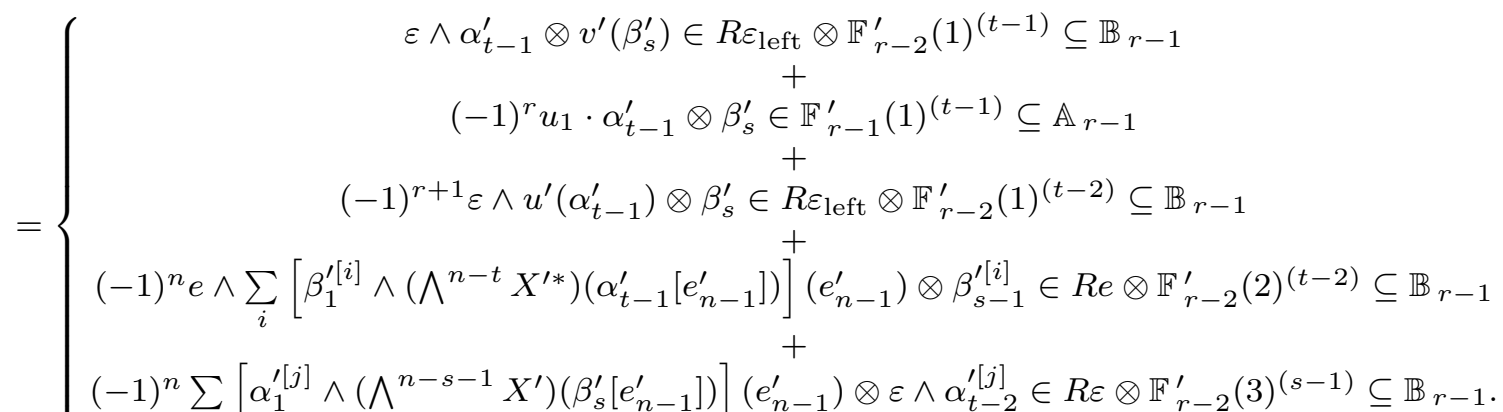


On the other hand, the right side of (4.14) is equal to

$$
\begin{gathered}
\varphi_{r-1}\left\{\begin{array}{c}
(-1)^{r} u_{1} \cdot \alpha_{t-1}^{\prime} \otimes \beta_{s}^{\prime} \in \mathbb{F}_{r-1}^{\prime}(1)^{(t-1)} \subseteq \widehat{\mathbb{A}}_{r-1} \\
\mathfrak{f}_{r-1}(1)\left(\alpha_{t-1}^{\prime} \otimes \beta_{s}^{\prime}\right) \in \widehat{\mathbb{B}}_{r-1}
\end{array}\right. \\
=\varphi_{r-1}\left\{\begin{array}{c}
(-1)^{r} u_{1} \cdot \alpha_{t-1}^{\prime} \otimes \beta_{s}^{\prime} \in \mathbb{F}_{r-1}^{\prime}(1)^{(t-1)} \subseteq \widehat{\mathbb{A}}_{r-1} \\
f_{r-1}^{\prime \prime}[1,1]\left(\alpha_{t-1}^{\prime} \otimes \beta_{s}^{\prime}\right) \in \mathbb{F}_{r-2}^{\prime}(1) \subseteq \widehat{\mathbb{B}}_{r-1} \\
+ \\
(-1)^{n-1} f_{r-1}^{\prime \prime}[2,1]\left(\alpha_{t-1}^{\prime} \otimes \beta_{s}^{\prime}\right) \in \mathbb{F}_{r-2}^{\prime}(2) \subseteq \widehat{\mathbb{B}}_{r-1} \\
(-1)^{n-1} f_{r-1}^{\prime \prime}[3,1]\left(\alpha_{t-1}^{\prime} \otimes \beta_{s}^{\prime}\right) \in \mathbb{F}_{r-2}^{\prime}(3) \subseteq \widehat{\mathbb{B}}_{r-1}
\end{array}\right. \\
=\left\{\begin{array}{c}
(-1)^{r} u_{1} \cdot \alpha_{t-1}^{\prime} \otimes \beta_{s}^{\prime} \in \mathbb{F}_{r-1}^{\prime}(1)^{(t-1)} \subseteq \mathbb{A}_{r-1} \\
+{ }_{r-1} \\
\text { nat } \circ f_{r-1}^{\prime \prime}[1,1]\left(\alpha_{t-1}^{\prime} \otimes \beta_{s}^{\prime}\right) \in R \varepsilon_{\text {left }} \otimes \mathbb{F}_{r-2}^{\prime}(1) \subseteq \mathbb{B}_{r-1} \\
(-1)^{n} \text { nat } \circ f_{r-1}^{\prime \prime}[2,1]\left(\alpha_{t-1}^{\prime} \otimes \beta_{s}^{\prime}\right) \in R e \otimes \mathbb{F}_{r-2}^{\prime}(2) \subseteq \mathbb{B}_{r-1} \\
+{ }_{r-1}^{\prime} \\
(-1)^{n} \text { nat } \circ f_{r-1}^{\prime \prime}[3,1]\left(\alpha_{t-1}^{\prime} \otimes \beta_{s}^{\prime}\right) \in R \varepsilon \otimes \mathbb{F}_{r-2}^{\prime}(3) \subseteq \mathbb{B}_{r-1} ;
\end{array}\right.
\end{gathered}
$$

thus, (4.14) holds in this case. In our third calculation, we take $y=\alpha_{t}^{\prime} \otimes \beta_{s-1}^{\prime}$ in $\mathbb{F}_{r-1}^{\prime}(1)^{(t)} \subseteq \widehat{\mathbb{C}}_{r}$. Let $\Delta\left(\alpha_{t}^{\prime}\right)=\sum_{j} \alpha_{1}^{\prime[j]} \otimes \alpha_{t-1}^{\prime[j]}$, and $\Delta\left(\beta_{s-1}^{\prime}\right)=\sum_{i} \beta_{1}^{\prime[i]} \otimes \beta_{s-2}^{\prime[i]}$. The left side of $(4.14)$ is equal to $f_{r}(1)^{(t)}\left((-1)^{r} \alpha_{t}^{\prime} \otimes \varepsilon \wedge \beta_{s-1}^{\prime}\right)$

$$
\begin{aligned}
& =(-1)^{r}\left\{\begin{array}{c}
\alpha_{t}^{\prime} \otimes v\left(\varepsilon \wedge \beta_{s-1}^{\prime}\right) \in \mathbb{F}_{r-1}(1)^{(t)} \\
+ \\
(-1)^{r} u\left(\alpha_{t}^{\prime}\right) \otimes \varepsilon \wedge \beta_{s-1}^{\prime} \\
+\mathbb{F}_{r-1}(1)^{(t-1)} \\
\sum_{i}-\left[\beta_{1}^{\prime[i]} \wedge\left(\wedge^{n-t} X^{*}\right)\left(\alpha_{t}^{\prime}\left[e_{n}\right]\right)\right]\left(e_{n}\right) \otimes \varepsilon \wedge \beta_{s-2}^{\prime[i]} \in \mathbb{F}_{r-1}(2)^{(t-1)} \\
+ \\
\sum_{j}\left[\alpha_{1}^{\prime[j]} \wedge\left(\bigwedge^{n-s} X\right)\left(\left[\varepsilon \wedge \beta_{s-1}^{\prime}\right]\left[e_{n}\right]\right)\right]\left(e_{n}\right) \otimes \alpha_{t-1}^{\prime[j]} \in \mathbb{F}_{r-1}(3)^{(s-1)}
\end{array}\right.
\end{aligned}
$$

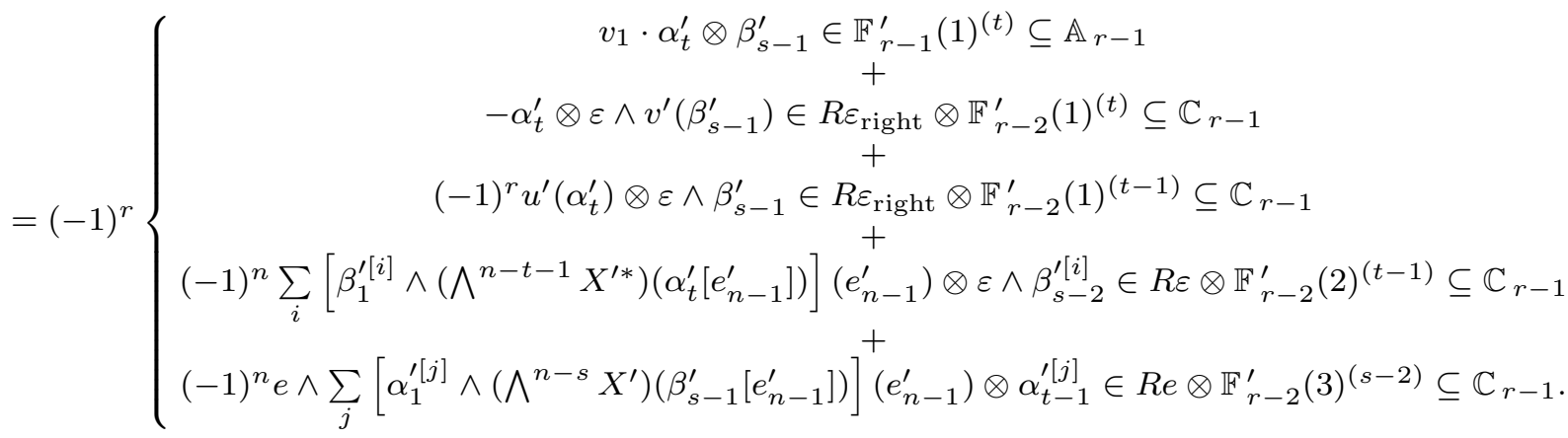

The right side of (4.14) is equal to

$$
\varphi_{r-1}\left\{\begin{array}{c}
(-1)^{r} v_{1} \cdot \alpha_{t}^{\prime} \otimes \beta_{s-1}^{\prime} \in \mathbb{F}_{r-1}^{\prime}(1)^{(t)} \subseteq \widehat{\mathbb{A}}_{r-1} \\
++\widehat{F}_{r-1}^{\prime \prime}[1,1]\left(\alpha_{t}^{\prime} \otimes \beta_{s-1}^{\prime}\right) \in \mathbb{F}_{r-2}^{\prime}(1) \subseteq \widehat{\mathbb{C}}_{r-1} \\
++{ }_{r-1}^{\prime} \\
(-1)^{n-1} f_{r-1}^{\prime \prime}[2,1]\left(\alpha_{t}^{\prime} \otimes \beta_{s-1}^{\prime}\right) \in \mathbb{F}_{r-2}^{\prime}(2) \subseteq \widehat{\mathbb{C}}_{r-1} \\
+
\end{array}\right.
$$




$$
=\left\{\begin{array}{c}
(-1)^{r} v_{1} \cdot \alpha_{t}^{\prime} \otimes \beta_{s-1}^{\prime} \in \mathbb{F}_{r-1}^{\prime}(1)^{(t)} \subseteq \mathbb{A}_{r-1} \\
+{ }_{r-1} \\
(-1)^{r-1} \text { nat } \circ f_{r-1}^{\prime \prime}[1,1]\left(\alpha_{t}^{\prime} \otimes \beta_{s-1}^{\prime}\right) \in R \varepsilon_{\text {right }} \otimes \mathbb{F}_{r-2}^{\prime}(1) \subseteq \mathbb{C}_{r-1} \\
+ \\
(-1)^{r+n} \text { nat } \circ f_{r-1}^{\prime \prime}[2,1]\left(\alpha_{t}^{\prime} \otimes \beta_{s-1}^{\prime}\right) \in R \varepsilon \otimes \mathbb{F}_{r-2}^{\prime}(2) \subseteq \mathbb{C}_{r-1} \\
+{ }^{+} \\
(-1)^{r-n} \text { nat } \circ f_{r-1}^{\prime \prime}[3,1]\left(\alpha_{t}^{\prime} \otimes \beta_{s-1}^{\prime}\right) \in \operatorname{Re} \otimes \mathbb{F}_{r-2}^{\prime}(3) \subseteq \mathbb{C}_{r-1}
\end{array}\right.
$$

thus, (4.14) holds in this case. In our fourth calculation, we take $y=\alpha_{t-1}^{\prime} \otimes \beta_{s-1}^{\prime}$ in $\mathbb{F}_{r-2}^{\prime}(1)^{(t-1)} \subseteq \widehat{\mathbb{D}}_{r}$. Let $\Delta\left(\alpha_{t-1}^{\prime}\right)=\sum_{j} \alpha_{1}^{\prime[j]} \otimes \alpha_{t-2}^{\prime[j]}$ and $\Delta\left(\beta_{s-1}^{\prime}\right)=\sum_{i} \beta_{1}^{\prime[i]} \otimes \beta_{s-2}^{\prime[i]}$.

The left side of (4.14) is equal to $f_{r}(1)^{(t)}\left((1)^{r} \varepsilon \wedge \alpha_{t-1}^{\prime} \otimes \varepsilon \wedge \beta_{s-1}^{\prime}\right)$

$$
=(1)^{r}\left\{\begin{array}{c}
\varepsilon \wedge \alpha_{t-1}^{\prime} \otimes v\left(\varepsilon \wedge \beta_{s-1}^{\prime}\right) \in \mathbb{F}_{r-1}(1)^{(t)} \\
+ \\
(-1)^{r} u\left(\varepsilon \wedge \alpha_{t-1}^{\prime}\right) \otimes \varepsilon \wedge \beta_{s-1}^{\prime} \in \mathbb{F}_{r-1}(1)^{(t-1)} \\
+ \\
-\sum_{i}\left[\beta_{1}^{\prime[i]} \wedge\left(\wedge^{n-t} X^{*}\right)\left(\left[\varepsilon \wedge \alpha_{t-1}^{\prime}\right]\left[e_{n}\right]\right)\right]\left(e_{n}\right) \otimes \varepsilon \wedge \beta_{s-2}^{\prime[i]} \in \mathbb{F}_{r-1}(2)^{(t-1)} \\
+ \\
{\left[\varepsilon \wedge\left(\wedge^{n-s} X\right)\left(\left[\varepsilon \wedge \beta_{s-1}^{\prime}\right]\left[e_{n}\right]\right)\right]\left(e_{n}\right) \otimes \alpha_{t-1}^{\prime} \in \mathbb{F}_{r-1}(3)^{(s-1)}} \\
+ \\
-\sum_{j}\left[\alpha_{1}^{\prime[j]} \wedge\left(\bigwedge^{n-s} X\right)\left(\left[\varepsilon \wedge \beta_{s-1}^{\prime}\right]\left[e_{n}\right]\right)\right]\left(e_{n}\right) \otimes \varepsilon \wedge \alpha_{t-2}^{\prime[j]} \in \mathbb{F}_{r-1}(3)^{(s-1)}
\end{array}\right.
$$

which is equal to $(-1)^{r}$ times

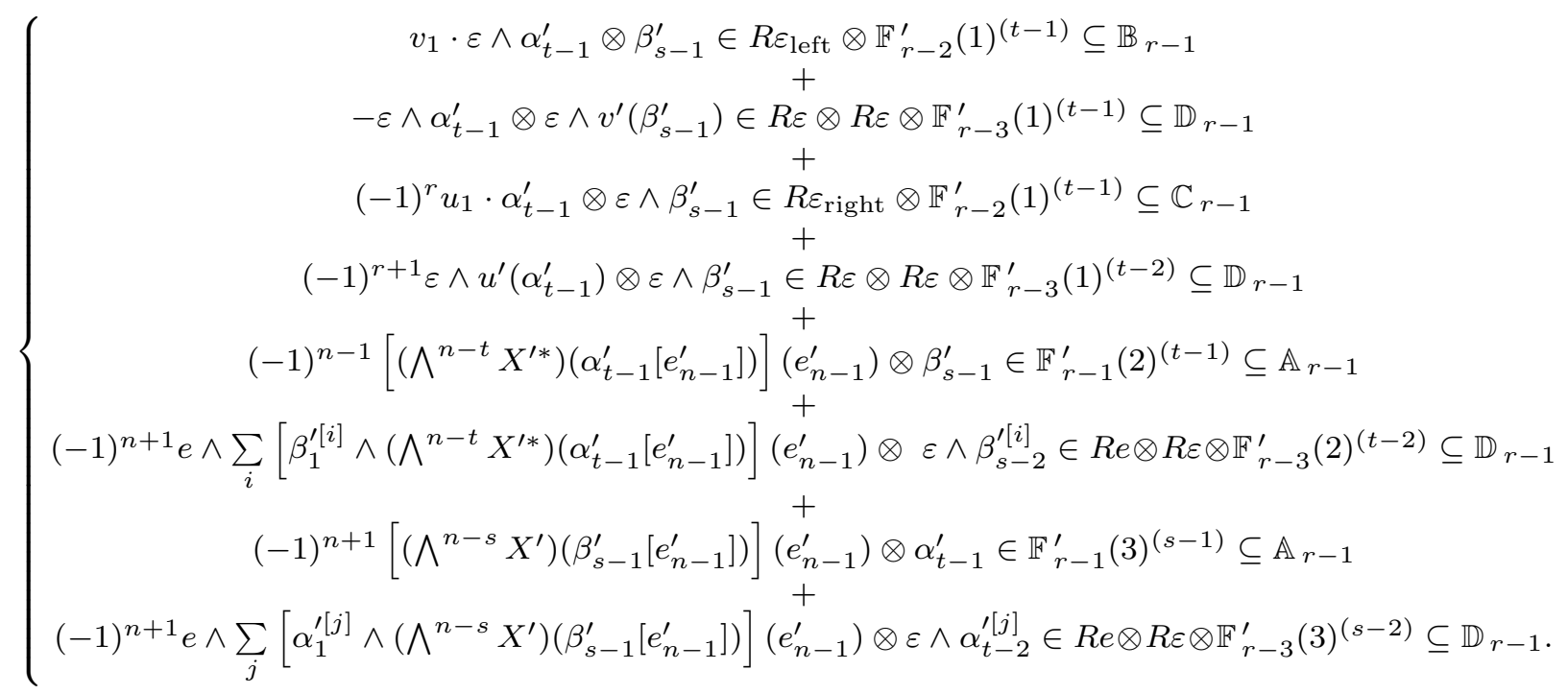

The right side of (4.14) is equal to

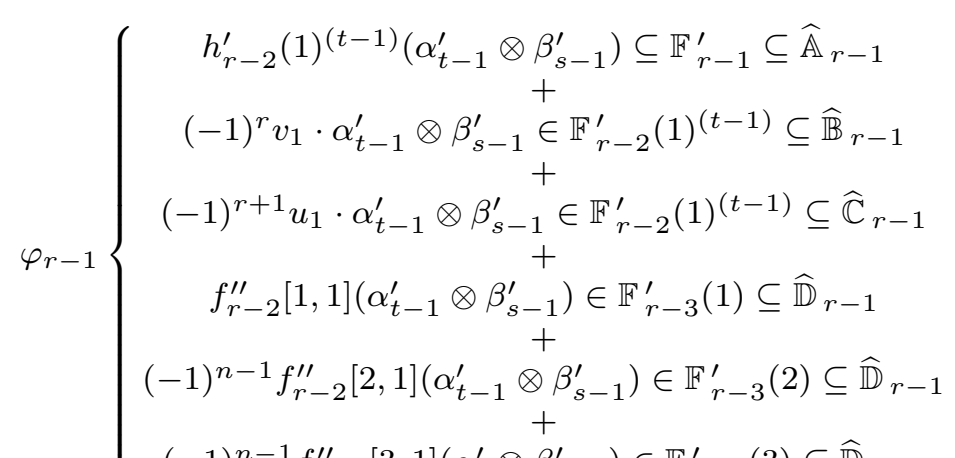




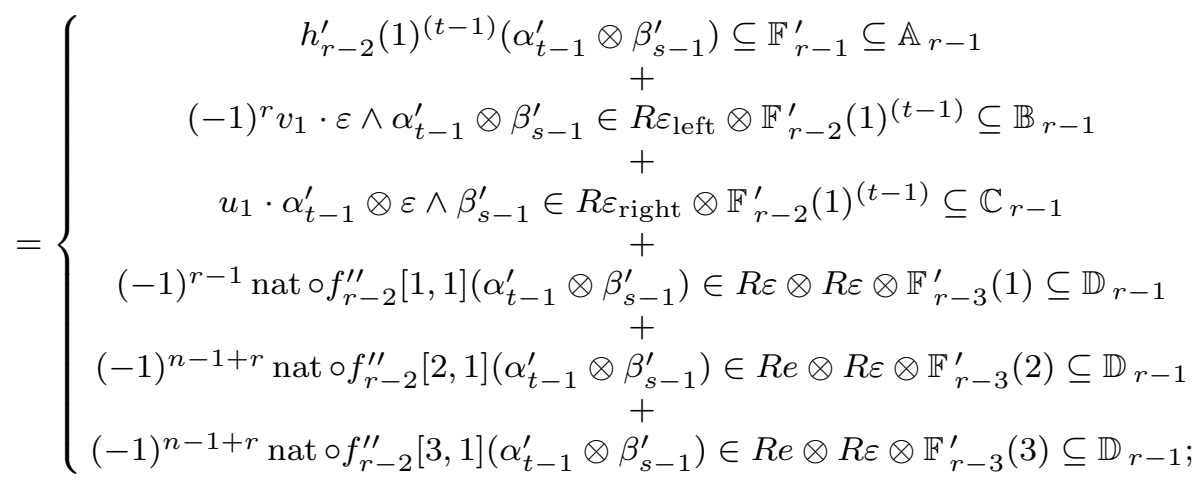

thus, (4.14) holds in this case.

\section{Further applications and questions.}

Data 5.1. Fix an integer $n$, with $3 \leq n$. Let $\mathbf{u}_{1 \times n}, \mathbf{X}_{n \times n}$, and $\mathbf{v}_{n \times 1}$ be matrices of indeterminates over a commutative noetherian ring $R_{0}, H$ be the ideal $H(\mathbf{u}, \mathbf{X}, \mathbf{v})$ of Definition 1.1 in the polynomial ring $R=R_{0}\left[\left\{u_{i}, v_{i}, x_{i j} \mid 1 \leq i, j \leq n\right\}\right]$, and $\bar{R}$ be the quotient $R / H$.

Theorem 5.2. Adopt Data 5.1. The ideal $H$ of $R$ is a perfect Gorenstein ideal of grade $2 n$.

Proof. Let $u, X, v$ be the data of 1.6 which is obtained from $\mathbf{u}, \mathbf{X}, \mathbf{v}$ by way of Convention 1.8, and let $\mathbb{M}$ be the complex $\mathbb{M}\left[u, X,(-1)^{\frac{n(n-1)}{2}} v\right]$. Theorems 4.1 and 3.1 show that $\mathbb{M}$ is a resolution of $\bar{R}$ of length $2 n$. It follows from [4] and Observation 3.19 that

$$
\begin{gathered}
\text { grade } H \leq \operatorname{pd}_{R} \bar{R} \leq 2 n \leq \text { grade } I_{1}\left(m_{2 n}\right)=\text { grade } H \quad \text { and } \\
\operatorname{Ext}_{R}^{2 n}(\bar{R}, R)=H_{0}\left(\mathbb{M}^{*}\right)=R / I_{1}\left(m_{2 n}\right)=\bar{R}
\end{gathered}
$$

Remark. There are at least two other ways to calculate grade $H$ : one can calculate the height of $H$ as in [10] (see also Lemma 5.3), or one can specialize $H$, along the lines of Example 5.8, and then calculate its grade.

Lemma 5.3. Adopt Data 5.1. Let $s$ be $u_{i}$ for some $i$, or $v_{i}$ for some $i$, or some $n-2$ minor of $X$. Then, there exist indeterminates $Y_{1}, \ldots Y_{n^{2}}$ such that $\bar{R}_{s}=$ $R_{0}\left[Y_{1}, \ldots Y_{n^{2}}\right]_{s}$.

Proof. Begin with $s=u_{1}$. It is not difficult to show that

$$
H_{s}=\left(I_{1}(\mathbf{u X})+\left(\left\{(\mathbf{v u}-\operatorname{Adj} \mathbf{X})_{i 1} \mid 1 \leq i \leq n\right\}\right)\right) R_{s}
$$

see, for example, [10, Proposition 3.3.2]. It follows that

$$
\bar{R}_{s}=R_{0}\left[\left\{x_{i j} \mid 2 \leq i \leq n, 1 \leq j \leq n\right\}, u_{1}, \ldots, u_{n}\right]_{s} .
$$

Now, we let $s$ be the determinant of the submatrix of $\mathbf{X}$ which is obtained by deleting rows and columns $n$ and $n-1$. Observe that

$H_{s}=\left(\left\{(\mathbf{u X X})_{i} \mid 1 \leq i \leq n-2\right\},\left\{(\mathbf{X v})_{i} \mid 1 \leq i \leq n-2\right\},\left\{(\mathbf{v u}-\operatorname{Adj} \mathbf{X})_{i j} \mid n-1 \leq i, j \leq n\right\}\right) R_{s} ;$ and therefore,

$\bar{R}_{s}=R_{0}\left[u_{n-1}, u_{n}, v_{n-1}, v_{n},\left\{x_{i j} \mid(i, j) \neq(n-1, n-1),(n-1, n),(n, n-1) \text { or }(n, n)\right\}\right]_{s}$. 
Corollary 5.4. Adopt Data 5.1.

(a) If $R_{0}$ is a domain, then so is $\bar{R}$.

(b) Let $k$ be an integer with $k \leq 8$.

(i) If $R_{0}$ satisfies the Serre condition $\left(S_{k+1}\right)$, then so does $\bar{R}$.

(ii) If $R_{0}$ satisfies the Serre conditions $\left(R_{k}\right)$ and $\left(S_{k+1}\right)$, then so does $\bar{R}$.

In particular, if the ring $R_{0}$ is reduced, then so is $\bar{R}$; if the ring $R_{0}$ is normal, then so is $\bar{R}$.

Proof. Assertion (a) follows from Theorem 5.2 together with [10, Proposition 3.3.2]. A version of this argument, which contains more details, may be found in [3, Theorem 2.10]. The proof of (b) also follows a standard argument; see, for example, [9, Theorem 9.4]. Let $P$ be a prime of $R$ with $H \subseteq P$ and depth $\bar{R}_{P} \leq k$. For (i) it suffices to show that $\bar{R}_{P}$ is Cohen-Macaulay; for (ii) it suffices to show that $\bar{R}_{P}$ is regular. Since $H R_{P}$ is a perfect ideal of grade $2 n$ in the ring $R_{P}$, we know (use [3, Proposition 16.18], if necessary) that

grade $P R_{P}-2 n=\operatorname{grade} P R_{P}-\operatorname{grade} H R_{P} \leq \operatorname{grade} \frac{P R_{P}}{H R_{P}}=\operatorname{depth} \bar{R}_{P} \leq k$.

It follows that grade $P \leq 2 n+8$; thus, $I_{1}(\mathbf{u})+I_{1}(\mathbf{v})+I_{n-2}(\mathbf{X})$ is not contained in $P$. It follows from Lemma 5.3 that $\bar{R}_{P}$ is a localization of a polynomial ring over the local ring $A=\left(R_{0}\right)_{P \cap R_{0}}$. The hypothesis $\operatorname{depth} \bar{R}_{P} \leq k$ ensures that $\operatorname{depth} A \leq k$. It follows that $A$ is Cohen-Macaulay in case (i) and regular in case (ii). The proof is complete.

Corollary 5.5. Assume that the ring $R_{0}$ of Data 5.1 is regular local with maximal ideal $\mathfrak{m}$. If $\mathfrak{M}$ is the maximal ideal $\mathfrak{m} R+I_{1}(\mathbf{u})+I_{1}(\mathbf{v})+I_{1}(\mathbf{X})$ of $R$, then the localization $\bar{R}_{\mathfrak{M}}$ is not in the linkage class of a complete intersection.

Proof. Corollary 5.4 shows that $\bar{R}_{\mathfrak{M}}$ satisfies the Serre condition $\left(R_{8}\right)$; however, Huneke and Ulrich [7, Theorem 4.2] have proved that a licci Gorenstein ring can satisfy $\left(R_{7}\right)$ only if it is a complete intersection.

Remark 5.6. A second proof of Corollary 5.5 is available when $R_{0}$ is a field and $n$ is odd. In this case, the graded twists

$$
0 \rightarrow \bigoplus_{j} R\left(-d_{g j}\right) \rightarrow \cdots \rightarrow \bigoplus_{j} R\left(-d_{1 j}\right) \rightarrow R
$$

of the minimal resolution of $\bar{R}$ satisfy the inequality

$$
\max _{j}\left\{d_{g j}\right\} \leq(\text { grade } H-1) \min _{j}\left\{d_{1 j}\right\}
$$

of [7, Cor. 5.13], because Remark 3.8 shows that $\max _{j}\left\{d_{g j}\right\}=n^{2}$, for $g=$ grade $H=$ $2 n$, and if we take $\operatorname{deg} u=\operatorname{deg} v=\frac{n-1}{2}$, then $\min _{j}\left\{d_{1 j}\right\}=\frac{n+1}{2}$.

The following consequence of Theorem 5.2 is an application of the principle of 
Corollary 5.7. Let $\mathbf{u}_{1 \times n}^{\prime}, \mathbf{X}_{n \times n}^{\prime}$, and $\mathbf{v}_{n \times 1}^{\prime}$ be matrices with entries from a commutative noetherian ring $R^{\prime}, H^{\prime}$ be the ideal $H\left(\mathbf{u}^{\prime}, \mathbf{X}^{\prime}, \mathbf{v}^{\prime}\right)$ of Definition $1.1, u^{\prime}, X^{\prime}, v^{\prime}$ be the Data of 1.6 constructed from $\mathbf{u}^{\prime}, \mathbf{X}^{\prime}, \mathbf{v}^{\prime}$ by way of Convention 1.8 , and $\mathbb{M}$ be the complex $\mathbb{M}\left[u^{\prime}, X^{\prime},(-1)^{\frac{n(n-1)}{2}} v^{\prime}\right]$. If $H^{\prime}$ is a proper ideal with $2 n \leq$ grade $H^{\prime}$, then $H^{\prime}$ is a perfect Gorenstein ideal of grade equal to $2 n$, and $\mathbb{M}$ is an $R^{\prime}$-resolution of $R^{\prime} / H^{\prime}$. Furthermore, if $R^{\prime}$ is a local ring with maximal ideal $\mathfrak{m}$ and the entries of $\mathbf{u}^{\prime}, \mathbf{X}^{\prime}$, and $\mathbf{v}^{\prime}$ are all in $\mathfrak{m}$, then $\mathbb{M}$ is the minimal resolution of $R^{\prime} / H^{\prime}$.

Example 5.8. Form the ideal $H^{\prime}=H\left(\mathbf{u}, \mathbf{X}^{\prime}, \mathbf{v}\right)$ using generic matrices $\mathbf{u}$ and $\mathbf{v}$ and a generic diagonal matrix $\mathbf{X}^{\prime}=\operatorname{diag}\left(x_{1}, \ldots, x_{n}\right)$. It is easy to see that $2 n \leq$ grade $H^{\prime}$; and therefore Corollary 5.7 guarantees that $H^{\prime}$ is a perfect Gorenstein ideal of grade $2 n$. A significant amount of computer experimentation preceded the discovery of the resolutions $\mathbb{F}$ and $\mathbb{M}$. Among all specializations of the generic Data 5.1 , the ideals of the present example, with $\operatorname{deg} u=\left[\left[\frac{n-1}{2}\right]\right]$, yield the best results when resolved using the computer program MACAULAY.

As our final application, we determine which of the relations in the minimal resolution $\mathbb{M}$ are Koszul relations on the generators of the defining ideal of $H_{0}(\mathbb{M})$. It is much easier to compute the subalgebra $k\left[\right.$ Tor $\left._{1}\right]$ than it is to compute the entire algebra structure of Tor. . Nonetheless, a significant amount of information is carried by this subalgebra; see, for example, [8].

Corollary 5.9. Adopt the notation and hypotheses of Corollary 5.7 with $3 \leq n$, $\left(R^{\prime}, \mathfrak{m}, k\right)$ a local ring, and the entries of $\mathbf{u}^{\prime}, \mathbf{X}^{\prime}$, and $\mathbf{v}^{\prime}$ all in $\mathfrak{m}$. Then the subalgebra $k\left[\operatorname{Tor}_{1}\right]$ of $\operatorname{Tor}_{\bullet}^{R^{\prime}}\left(R^{\prime} / H^{\prime}, k\right)$, which is generated by Tor $_{1}$, is isomorphic to the following quotient of the exterior algebra $\bigwedge_{k}^{\bullet} k^{n^{2}+2 n}$ :

$$
k\left[\mathrm{Tor}_{1}\right] \cong \frac{\bigwedge_{k}^{\bullet}\left(V_{1} \oplus V_{2} \oplus V_{3}\right)}{\left(\bigwedge^{n-1} V_{2}+\bigwedge^{n-1} V_{3}+\bigwedge^{2} V_{1}+V_{1} V_{2}+V_{1} V_{3}+V_{2} V_{3}\right)}
$$

where $V_{1}, V_{2}$, and $V_{3}$ are vector spaces over $k$ with $\operatorname{dim} V_{1}=n^{2}$ and $\operatorname{dim} V_{2}=$ $\operatorname{dim} V_{3}=n$. Furthermore, there is an algebra embedding

$$
k\left[\operatorname{Tor}_{1}\right] \ltimes\left(\operatorname{Hom}_{k}\left(k\left[\operatorname{Tor}_{1}\right], k\right)[-2 n]\right) \hookrightarrow \operatorname{Tor}_{\bullet}^{R^{\prime}}\left(R^{\prime} / H^{\prime}, k\right) .
$$

Proof. The final assertion reflects the fact that $\operatorname{Tor}_{\bullet}^{R^{\prime}}\left(R^{\prime} / H^{\prime}, k\right)$ is a Poincaré algebra of length $2 n$ (see [6, Theorem 1.5] or [1, Example 9.4]) and $k\left[\text { Tor }_{1}\right]_{n}=0$. To complete the proof, we calculate $k\left[\right.$ Tor $\left._{1}\right]$. Let - represent the functor $-\otimes_{R^{\prime}} k$. If $\gamma: \bigwedge^{\bullet} \mathbb{M}_{1} \rightarrow \mathbb{F}$ is any map of complexes which extends the commutative diagram

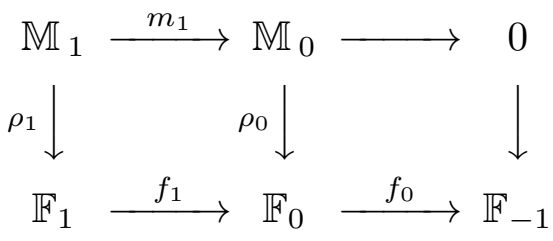

of Proposition $3.14(\mathrm{~d})$, and $\psi: \mathbb{F} \rightarrow \mathbb{M}$ is the map of complexes from Definition $3.12(\mathrm{~b})$, then the composition 
induces an isomorphism

$$
k\left[\operatorname{Tor}_{1}\right] \cong \frac{\bigwedge^{\bullet} \overline{\mathbb{M}_{1}}}{\operatorname{Ker} \bar{\psi} \circ \bar{\gamma}} .
$$

Recall that $\mathbb{M}_{1}=\mathbb{F}_{1}(1)^{(1)} \oplus \mathbb{F}_{1}(2)^{(1)} \oplus \mathbb{F}_{1}(3)^{(1)}$. We define the relevant parts of $\gamma$ as follows:

(a) Let $r$ be an integer with $1 \leq r, a_{1}^{[1]} \otimes 1, \ldots, a_{1}^{[r]} \otimes 1$ be elements from $\mathbb{F}_{1}(3)^{(1)}$, and $a_{r}$ be the element $a_{1}^{[1]} \wedge \ldots \wedge a_{1}^{[r]}$ of $\bigwedge^{r} F$. The map $\gamma_{r}: \bigwedge^{r} \mathbb{F}_{1}(3)^{(1)} \rightarrow \mathbb{F}_{r}$ is given by

$$
\gamma_{r}\left(\left(a_{1}^{[1]} \otimes 1\right) \wedge \ldots \wedge\left(a_{1}^{[r]} \otimes 1\right)\right)=\left\{\begin{array}{c}
1 \otimes\left(\bigwedge^{r} X^{*}\right)\left(a_{r}\right) \in \mathbb{F}_{r}(2)^{(0)} \\
+ \\
a_{r} \otimes 1 \in \mathbb{F}_{r}(3)^{(r)}
\end{array}\right.
$$

(b) Let $r$ be an integer with $1 \leq r, a_{1}^{[1]} \otimes 1, \ldots, a_{1}^{[r]} \otimes 1$ be elements from $\mathbb{F}_{1}(2)^{(1)}$, and $a_{r}$ be the element $a_{1}^{[1]} \wedge \ldots \wedge a_{1}^{[r]}$ of $\bigwedge^{r} F$. The map $\gamma_{r}: \wedge^{r} \mathbb{F}_{1}(2)^{(1)} \rightarrow \mathbb{F}_{r}$ is given by

$$
\gamma_{r}\left(\left(a_{1}^{[1]} \otimes 1\right) \wedge \ldots \wedge\left(a_{1}^{[r]} \otimes 1\right)\right)=\left\{\begin{array}{c}
(-1)^{\frac{r(r-1)}{2}} a_{r} \otimes 1 \in \mathbb{F}_{r}(2)^{(r)} \\
+\quad \begin{array}{c}
{ }^{(r)} \\
(-1)^{\frac{r(r-1)}{2}} 1 \otimes\left(\bigwedge^{r} X\right)\left(a_{r}\right) \in \mathbb{F}_{r}(3)^{(0)}
\end{array}
\end{array}\right.
$$

(c) If $\alpha_{1} \otimes \beta_{1} \in \mathbb{F}_{1}(1)^{(1)}$ and $a_{1} \otimes 1 \in \mathbb{F}_{1}(2)^{(1)}$, then

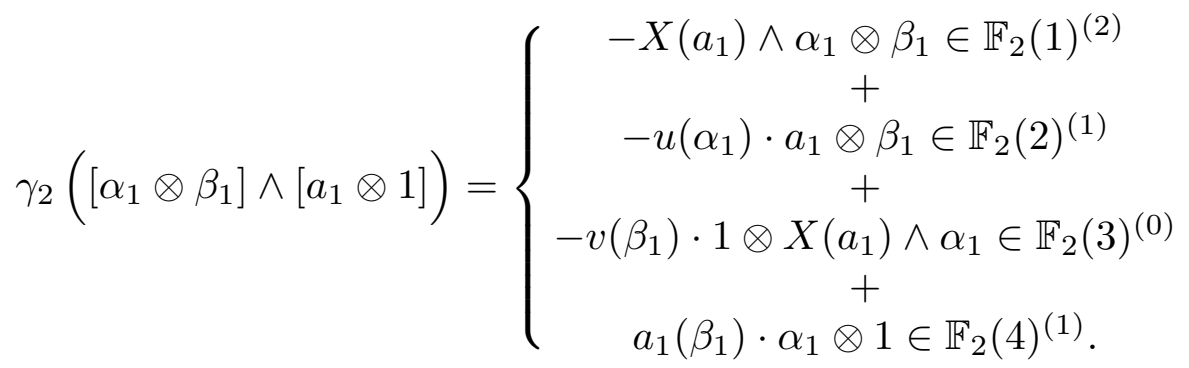

(d) If $\alpha_{1} \otimes \beta_{1} \in \mathbb{F}_{1}(1)^{(1)}$ and $a_{1} \otimes 1 \in \mathbb{F}_{1}(3)^{(1)}$, then

$$
\gamma_{2}\left(\left[\alpha_{1} \otimes \beta_{1}\right] \wedge\left[a_{1} \otimes 1\right]\right)=\left\{\begin{array}{c}
-\alpha_{1} \otimes X^{*}\left(a_{1}\right) \wedge \beta_{1} \in \mathbb{F}_{2}(1)^{(1)} \\
+\quad X_{1} \in \mathbb{F}_{2}(2)^{(0)} \\
u\left(\alpha_{1}\right) \cdot 1 \otimes X^{*}\left(a_{1}\right) \wedge \beta_{1}+a_{1} \in \mathbb{F}_{2}(3)^{(1)} \\
v\left(\beta_{1}\right) \cdot a_{1} \otimes \alpha_{1} \\
+ \\
a_{1}\left(\alpha_{1}\right) \cdot 1 \otimes \beta_{1} \in \mathbb{F}_{2}(4)^{(0)} .
\end{array}\right.
$$

(e) If $a_{1} \otimes 1 \in \mathbb{F}_{1}(2)^{(1)}$ and $b_{1} \otimes 1 \in \mathbb{F}_{1}(3)^{(1)}$, then

$$
\gamma_{2}\left(\left[a_{1} \otimes 1\right] \wedge\left[b_{1} \otimes 1\right]\right)=\left\{\begin{array}{c}
-a_{1} \otimes X^{*}\left(b_{1}\right) \in \mathbb{F}_{2}(2)^{(1)} \\
+ \\
-b_{1} \otimes X\left(a_{1}\right) \in \mathbb{F}_{2}(3)^{(1)}
\end{array}\right.
$$

(f) The restriction of $\gamma_{2}$ to $\bigwedge^{2} \mathbb{F}_{1}(1)^{(1)}$ is the composition 
where $s$ is any splitting of the canonical map $\mathbb{F}_{1}(1)^{(1)} \otimes \mathbb{F}_{1}(1)^{(1)} \rightarrow \bigwedge^{2} \mathbb{F}_{1}(1)^{(1)}$, and

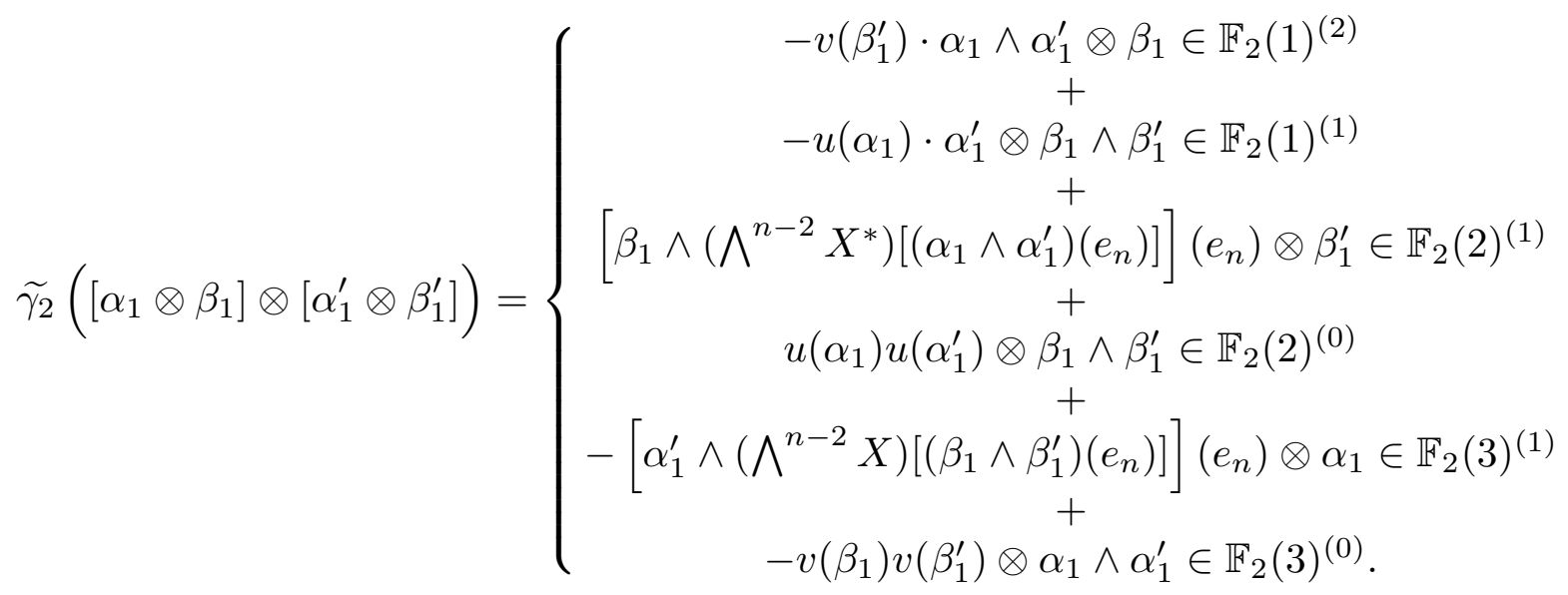

It is not difficult to see that the part of $\gamma$ which we have defined may be extended to give a map of complexes $\gamma: \bigwedge^{\bullet} \mathbb{M}_{1} \rightarrow \mathbb{F}$ which extends (5.10). It is also easy to see that the kernel of $\bar{\psi} \circ \bar{\gamma}$ is equal to

$\bigwedge^{n-1} \overline{\mathbb{F}_{1}(2)^{(1)}}+\bigwedge^{n-1} \overline{\mathbb{F}_{1}(3)^{(1)}}+\bigwedge^{2} \overline{\mathbb{F}_{1}(1)^{(1)}}+\overline{\mathbb{F}_{1}(1)^{(1)}} \otimes \overline{\mathbb{F}_{1}(2)^{(1)}}+\overline{\mathbb{F}_{1}(1)^{(1)}} \otimes \overline{\mathbb{F}_{1}(3)^{(1)}}+\overline{\mathbb{F}_{1}(2)^{(1)}} \otimes \overline{\mathbb{F}_{1}(3)^{(1)}}$,

and the proof is complete.

We conclude by recording some questions, which have not yet been addressed, about the ring $\bar{R}$ of Data 5.1. In this discussion we take $R_{0}=k$ to be the field of complex numbers. Is $\bar{R}$ a Unique Factorization Domain? If not, what is its divisor class group? Is $\bar{R}$ rigid? What is the cotangent cohomology module $T^{2}(\bar{R} / k, \bar{R})=\operatorname{Ext} \frac{1}{R}\left(H / H^{2}, \bar{R}\right)$ ? Does the minimal resolution of $\bar{R}$ admit the structure of a differential graded algebra? What is the rest of the algebra structure of $\operatorname{Tor}_{\bullet}^{R}(\bar{R}, k) ?$ Is the Poincaré series

$$
P \frac{k}{R}(z)=\sum_{i=0}^{\infty} \operatorname{dim}_{k} \operatorname{Tor}_{\bar{R}}(k, k) z^{i}
$$

a rational function? Are there interesting ideals, which are analogous to $H$, when the matrix $\mathbf{X}$ is not square?

\section{REFERENCES}

1. L. Avramov, Small homomorphisms of local rings, J. Alg. 50 (1978), 400-453.

2. H. Bass, On the ubiquity of Gorenstein rings, Math. Z. 82 (1963), 8-28.

3. W. Bruns and U. Vetter, Determinantal rings, Lecture Notes in Mathematics 1327, Springer Verlag, Berlin Heidelberg New York, 1988.

4. D. Buchsbaum and D. Eisenbud, What makes a complex exact?, J. Alg. 25 (1973), 259-268.

5. D. Buchsbaum and D. Eisenbud, Generic free resolutions and a family of generically perfect ideals, Advances Math. 18 (1975), 245-301.

6. D. Buchsbaum and D. Eisenbud, Algebra structures for finite free resolutions, and some structure theorems for ideals of codimension 3, Amer. J. Math. 99 (1977), 447-485.

7. C. Huneke and B. Ulrich, The structure of linkage, Annals of Math. 126 (1987), 277-334.

8. A. Kustin, Classification of the Tor-algebras of codimension four almost complete intersections, Trans. Amer. Math. Soc. 339 (1993), 61-85.

9. A. Kustin and B. Ulrich, A family of complexes associated to an almost alternating map, with applications to residual intersections, Mem. Amer. Math. Soc. 95 (1992), 1-94.

10. W. Vasconcelos, On the equations of Rees algebras, J. reine angew. Math. 418 (1991), 189- 